\title{
SOBOLEV SPACES AND HARMONIC MAPS FOR METRIC SPACE TARGETS
}

\author{
NICHOLAS J. KOREVAAR ${ }^{1}$ AND RICHARD M. SCHOEN ${ }^{2}$
}

\section{INTRODUCTION}

When one studies variational problems for maps between Riemannian manifolds one must consider spaces which we denote $W^{1, p}(\Omega, X)$. Here $\Omega$ is a compact domain in a Riemannian manifold, $X$ is a second Riemannian manifold, $p \in[1, \infty)$, and $W^{1, p}$ indicates that the first derivatives of the map are $L^{p}(\Omega)$. For $p>n$ such maps will be continuous, and the corresponding space $W^{1, p}(\Omega, X)$ can be given the structure of a smooth Banach manifold. This is because, for $p>n$, any map which is close in $W^{1, p}$ distance to a map $u_{0}$ can be described as a pointwise small deformation of $u_{0}$. This linear space of $W^{1, p}$ deformations is then a Banach space on which one can locally model $W^{1, p}(\Omega, X)$. For $p \leq n$ this is no longer possible, and the definition of the space $W^{1, p}(\Omega, X)$ becomes much less clear. This problem was first encountered by C.B. Morrey [Mo] in case $n=\operatorname{dim} \Omega=2$ and $p=2$. A great deal of effort was spent by Morrey to give a definition of this space. In more recent times people have exploited the embedding theorem of J. Nash, and considered $X$ to be a smooth submanifold of a Euclidean space $\mathbb{R}^{K}$. If we define $W^{1, p}(\Omega, X)$ to be the subset of the Banach space $W^{1, p}\left(\Omega, \mathbb{R}^{K}\right)$ consisting of those maps with image essentially in $X$, it turns out that this gives a workable definition for many purposes. An aesthetic drawback of this definition is that the space $W^{1, p}(\Omega, X)$ should depend only on the metric of $X$ and not on the embedding of $X$ into $\mathbb{R}^{K}$. A much more serious difficulty arises if one attempts to consider maps to spaces $X$ which are not smooth Riemannian manifolds. These

(1) Supported by NSF Grant DMS 9208666

(2) Supported by NSF Grant DMS 9208815 
could include Riemannian spaces with singularities, smooth Finsler manifolds, or infinite-dimensional spaces. In the first chapter of this paper we develop a direct intrinsic approach to this problem, and define $W^{1, p}(\Omega, X)$ for $(X, d)$ any complete metric space. This is shown to reduce to the usual space $W^{1, p}(\Omega)$ for $X=\mathbb{R}$, and reduces to the space described above for $X$ a smooth compact Riemannian manifold. We also derive the results which are essential for doing variational theory. These include lower semicontinuity of the $p$-energy, Rellich-type compactness results, and an $L^{p}$-trace theory for restrictions of maps to hypersurfaces.

To illustrate the idea of the Sobolev space theory, assume for simplicity of notation that $\Omega \subset \mathbb{R}^{n}$ is a Euclidean domain. If $u: \Omega \rightarrow X$ is a map, $x \in \Omega$, and $V \in \mathbb{R}^{n}$, we can give a formal definition of the norm of the directional derivative of $u$ in the direction $V$ at $x$ by

$$
\left|u_{*}(V)\right|=\lim _{\varepsilon \rightarrow 0}\left(\frac{d(u(x), u(x+\varepsilon V))}{\varepsilon}\right) .
$$

To define the $p$-Sobolev energy we can raise the distance quotient to the $p^{\text {th }}$ power, integrate over the unit vectors $V \in S^{n-1}$ and let

$$
e_{\varepsilon}(x)=\int_{S^{n-1}}\left(\frac{d(u(x), u(x+\varepsilon V)}{\varepsilon}\right)^{p} d \sigma(V) .
$$

The $p$-energy density $e(x)$ is then expected to be the limit as $\varepsilon \rightarrow 0$ of $e_{\varepsilon}(x)$. One of the main results of $\S 1$ is that the measures $e_{\varepsilon}(x) d x$ converge (weakly) in an almost monotone fashion as $\varepsilon \rightarrow 0$ provided that their total masses are uniformly bounded. Further it is shown that for $p>1$ the limiting measure is absolutely continuous with respect to Lebesgue measure, and hence may be written as $e(x) d x$ for an $L^{1}$ function $e(x)$. This convergence result may be considered as an analogue to the definition of the length of a continuous curve $\gamma:[0,1] \rightarrow X$ where $X$ is a metric space. The monotone property of the $e_{\varepsilon}(x) d x$ is analogous to the property that the approximation to the length given by

$$
\sum_{i=1}^{m} d\left(\gamma\left(x_{i}\right), \gamma\left(x_{i-1}\right)\right)
$$

is increased when the partition $\left\{x_{0}, \ldots, x_{m}\right\}$ is refined. The proof of the monotone property relies only on the triangle inequality and judicious changes 
of variable. The convergence of the measures $e_{\varepsilon}(x) d x$ seems to be essential in deriving any reasonable definition of the space $W^{1, p}(\Omega, X)$. If we were forced to choose a subsequence $\varepsilon_{i} \rightarrow 0$, we would not be able to show for example that a map which minimizes energy on $\Omega$ is also a minimizer on any subdomain of $\Omega$. The absolute continuity of the energy is also important in many further results. Both of these properties are used heavily in the analysis of energy minimizing maps.

We have not been able to find many references for earlier work in this area. H. Federer $[\mathrm{Fe}]$ discusses differentiability theory for maps of the real line into Banach Spaces. The idea of defining energy (at least for Lipschitz maps) in the way we have described has been around for some time. It was first proposed to the second author by S. Kerckhoff in the early 1980's. More recently, M. Gromov also proposed to use such a definition to study harmonic maps into metric spaces of non-positive curvature. As far as we know the results concerning the convergence of the $e_{\varepsilon}(x) d x$ to a limit energy measure are new, however, even for Lipschitz maps.

In $\S 2$ we construct least energy maps $(p=2)$ with prescribed values on $\partial \Omega$, into Alexandrov spaces $(X, d)$ of non-positive curvature. (The curvature condition is given in terms of triangle comparisons, see (2.1) for the precise definition.) Here an Alexandrov space is a complete metric space for which any pair of points can be joined by a curve whose length is equal to the distance between the points. The idea of using triangle (or quadrilateral) comparisons to define curvature bounds in metric spaces seems to be due to an Austrian mathematician A. Wald in the 1930's [Wa]. It has been developed by a Russian school of mathematicians centered around A.D. Alexandrov, starting in the late 1940 's.

We are able to construct (unique) least-energy maps for the Dirichlet problem because the energy satisfies a strong convexity property under the nonpositive curvature assumption. We do not require the space $X$ to be locally compact. We show that the minimizing map is Lipschitz in the interior of $\Omega$, with the Lipschitz constant locally bounded in terms of the total energy and the distance to $\partial \Omega$. The proof of continuity given here involves a rough form of the Eells-Sampson Bochner formula [ES]. The boundary continuity of 
a minimizing map to a metric space of non-positive curvature has been obtained recently by T. Serbinowski [Se]. His result says that the minimizing map is $C^{\alpha}$ up to the boundary for any $\alpha<1$, provided that the boundary map is also $C^{\alpha}$.

An important property which is needed to further advance the theory of harmonic maps is derived in $\S 2.3$. This is the result that any finite energy map into a space of non-positive curvature has an induced distance function which yields an infinitesimally Riemannian metric. Thus one can write the usual (trace) formula for energy density that holds for maps to smooth Riemannian manifolds. Note that for a general metric space target $X$ the induced metric will only be Finsler.

In $§ 2.5$ we develop some general averaging methods for maps into nonpositively curved spaces. We quantify the general principle that averaging decreases energy. We then apply these results to the study of homotopy and equivariant mapping problems. Precisely, we consider the existence problem for equivariant maps defined on the universal covering $\tilde{M}$ of a complete Riemannian manifold $M$, into a non-positively curved metric space $X$. These maps are assumed to be equivariant for a given homomorphism $\rho: \Gamma \rightarrow$ $\operatorname{Isom}(X)$, where $\Gamma=\pi_{1}(M)$. Assuming $\Gamma$ is finitely generated we construct a locally Lipschitz equivariant map with local Lipschitz constant bounded in terms of the infimum of the "translation function" $\delta: X \rightarrow \mathbb{R}^{+}$(see 2.6iii). If $M$ is compact, this produces a finite energy equivariant map with a sharp bound on its total energy. We then use the local Dirichlet problem together with a delicate averaging argument to construct a uniformly locally Lipschitz minimizing sequence. We prove convergence of minimizing sequences in case the domain has a nonempty boundary, or when the maps are lifts of maps between compact spaces.

The theory of harmonic maps into smooth manifolds of non-positive curvature begins with the work of J.Eells and H. Sampson [ES], and for manifolds with boundary with $\mathrm{R}$. Hamilton [Ha]. These theories were developed using the heat equation method. A variational approach to these results exploiting the energy convexity was given by the second author [Sch]. This was extended by him for maps into locally compact polyhedral spaces of non-positive cur- 
vature, and published jointly with Gromov [GS] ( $c f$. explanation in introduction to [GS]), along with certain applications to rigidity questions for discrete groups. Our work here is a strong generalization of these results.

The present paper is the first of three which we are writing in this area. In the second paper we will discuss some infinite dimensional applications. We will give a new characterization of Kazhdan's property $T$ for finitely-generated groups, and a generalization to actions on metric spaces. This can be used to derive property $T$ (and more general fixed-point results) using vanishing theorems. An $L^{2}$ version of the cocycle superrigidity theorem of R. Zimmer [Zi] follows in a natural way. (See also Corlette-Zimmer [CZ].) Of course, the harmonic map method provides a geometric means for studying actions of larger classes of discrete groups, not only those lattices which are superrigid.

A third paper discusses more refined properties of harmonic (i.e locally least energy) maps into arbitrary spaces of non-positive curvature. We will also include important monotonicity and tangent cone properties of the images of harmonic maps. Finally we will extend the theory developed in [GS] for maps to Euclidean buildings to include the case of non-locally compact buildings.

Acknowledgement. Both authors would like to thank the Institute for Advanced Study for its support. This paper was completed while they were visitors, during the academic year 1992-93. We thank Bruce Kleiner for several helpful discussions concerning the geometry of metric spaces.

Note added in proof. After this paper was written we received a preprint from J. Jost in which he also obtains some existence results for harmonic maps to (NPC) spaces.

\section{Contents}

Introduction

1. Sobolev Space Theory for Maps to Metric Spaces

1.1. Preliminary definitions

1.2. Approximate energies

1.3. The functionals ${ }_{\nu} E_{\varepsilon}$

1.4. Functional analysis lemmas 
1.5. The energy-density measure 580

1.6. Lower semicontinuity, and consistency when $X=\mathbb{R} \quad 581$

1.7. Directional energies 585

1.8. The calculus of directional energies 586

1.9. Differentiability theory for directional energies 591

1.10. Absolute continuity of $d e$ for $p>1 \quad 601$

1.11. The calculus of energy-density functions 603

1.12. Trace theory for Lipschitz domains 605

$\begin{array}{ll}\text { 1.13. Precompactness } & 612\end{array}$

2. Harmonic Maps into Non-Positively Curved Metric Spaces 614

2.1. Non-positively curved metric spaces 616

2.2. The solution to the Dirichlet Problem 622

$\begin{array}{ll}\text { 2.3. The pull-back inner product } \pi & 624\end{array}$

2.4. Geodesic homotopies and interior Lipschitz continuity 628

$\begin{array}{ll}\text { 2.5. Center of mass constructions } & 639\end{array}$

2.6. Equivariant mapping problems 643

$\begin{array}{ll}\text { 2.7. Homotopy problems } & 656\end{array}$

$\begin{array}{lc}\text { References } & 658\end{array}$

\section{Sobolev Space Theory for Maps to Metric Spaces}

In this chapter we construct the spaces $W^{1, p}(\Omega, X)$ (for $p>1$ ) and the space $B V(\Omega, X)$ (for $p=1$ ), in case $\left(\Omega^{n}, g\right)$ is a Riemannian domain and $(X, d)$ is a complete metric space. We only use the triangle inequality in the target to define Sobolev maps, much as one does when studying rectifiable curves into metric spaces. In fact, our approach represents a higher-dimensional (and higher $p$ ) generalization of curve theory.

We recall the definition of $L^{p}(\Omega, X)$ in $\S 1.1$. For fixed $u \in L^{p}(\Omega, X)$ we construct an approximate energy-density function $e_{\varepsilon}(x)$, by using the distance function $d$ to measure average displacements of $u$ in $\varepsilon$ - neighborhoods about $x$. We average symmetrically, so that if $X=\mathbb{R}$ and $u$ is smooth, then $e_{\varepsilon}(x) \rightarrow$ $c_{n, p}|\nabla u(x)|^{p}$ as $\varepsilon \rightarrow 0$. For technical reasons it is convenient to work with 
various symmetric averages: In (1.2ii) we first define $e_{\varepsilon}(x)$ essentially as in the introduction, taking it to be an average of $d^{p}(u(x), u(y))$, where $y$ is in the $\varepsilon$-sphere $S(x, \varepsilon)$ about $x$. Then for suitable measures $\nu$ on the interval $(0,2)$ we define ${ }_{\nu} e_{\varepsilon}(x)$ to be averages (with respect to $d \nu(\lambda)$ ) of the spherical averages $e_{\lambda \varepsilon}(x)$. Although this process is technically useful, it adds a layer of complication to our arguments. In order to focus on the main idea used to construct the Sobolev energy measure, we will restrict ourselves in this overview to a Euclidean domain and to the choice of $\nu$ (1.2vii) which leads to ball averages:

$$
\tilde{e}_{\varepsilon}(x)=(n+p) \int_{B(x, \varepsilon)} \frac{d^{p}(u(x), u(y))}{\varepsilon^{p}} \frac{d y}{\varepsilon^{n}} .
$$

The $\tilde{e}_{\varepsilon}(x)$ are bounded continuous functions (away from $\partial \Omega$ ), and the corresponding measures $e_{\varepsilon}(x) d x$ define linear functionals $\tilde{E}_{\varepsilon}(f)$ for $f \in C_{c}(\Omega)$, via integration. We say that $u \in L^{p}(\Omega, X)$ has finite energy $E$ whenever

$$
\sup _{\substack{0 \leq f \leq 1 \\ f \in C_{c}(\Omega)}} \limsup _{\varepsilon \rightarrow 0} \tilde{E}_{\varepsilon}(f) \equiv E<\infty .
$$

In this case we write $u \in W^{1, p}(\Omega, X)$ if $p>1$, or $u \in B V(\Omega, X)$ if $p=1$. (See 1.3).) For such $u$ we show that

$$
\lim _{\varepsilon \rightarrow 0} \tilde{E}_{\varepsilon}(f) \equiv E(f)
$$

exists for each $f \in C_{c}(\Omega)$. The key idea in this step is a "refinement lemma" (Lemma 1.3.1) which generalizes the fact that approximate lengths of curves increase when a partition is refined. Since the functional $E$ is linear and bounded (1.0ii) it follows from the Riesz representation theorem that there is an energy-density measure $d e$ for the map $u$, with $e_{\varepsilon} d \mu_{g} \rightarrow d e$ weakly, and with $e(\Omega)=E$. We sketch the refinement lemma and its consequences here, for the particular approximate energy functions (1.0i) and a Euclidean domain. For $f \in C_{c}(\Omega)$ with $f \geq 0$, and $\varepsilon>0$ we define the slightly larger function

$$
f_{\varepsilon}(x)=f(x)+\omega(f, \varepsilon)(x) .
$$

Here $\omega(f, \varepsilon)(x)$ is the oscillation of $f$ in $B(x, \varepsilon)$ (1.3iii). For small $\varepsilon>0$, $f_{\varepsilon} \in C_{c}(\Omega)$. Now, let any finite partition of the interval $[0,1]$ be given, into 
subintervals of lengths $\lambda_{i}, i=1, \ldots, m$. The refinement lemma is the inequality

$$
\tilde{E}_{\varepsilon}(f)^{1 / p} \leq \sum_{i} \lambda_{i}\left(\tilde{E}_{\lambda_{i} \varepsilon}\left(f_{\varepsilon}\right)\right)^{1 / p} .
$$

This inequality is a direct consequence of the iterated $(X, d)$ and $L^{p}$ triangle inequalities, as the following argument shows. We may write

$$
\tilde{E}_{\varepsilon}(f)=(n+p) \iint_{|x-y|<\varepsilon} f(x) \frac{d^{p}(u(x), u(y))}{\varepsilon^{p}} \frac{d y d x}{\varepsilon^{n}} .
$$

For small $\varepsilon, x$ in the support of $f$ and $|y-x|<\varepsilon$ we may partition the segment $x y$ into pieces of length $\lambda_{i}|x-y|$, and call the corresponding partition

$$
x=x_{0}, x_{1}, \ldots x_{m}=y .
$$

The $(X, d)$ triangle inequality implies

$$
d(u(x), u(y)) \leq \sum_{i=1}^{m} d\left(u\left(x_{i-1}\right), u\left(x_{i}\right)\right) .
$$

Thus from the iterated $L^{p}$ triangle inequality we have

(1.0v) $\tilde{E}_{\varepsilon}(f)^{1 / p} \leq \sum_{i}\left((n+p) \int_{|x-y|<\varepsilon} f(x) \frac{d^{p}\left(u\left(x_{i-1}\right), u\left(x_{i}\right)\right)}{\varepsilon^{p}} \frac{d y d x}{\varepsilon^{n}}\right)^{1 / p}$.

For each $i=1, \ldots, m$ we change variables in the corresponding integral, from $(x, y)$ to $\left(x_{i-1}, x_{i}\right)$. Write

$$
\mu_{i}=\sum_{j=1}^{i} \lambda_{j}
$$

Then we have the estimates

$$
\begin{aligned}
& d x d y=\frac{d x d x_{i}}{\mu_{i}^{n}}=\left(\frac{\mu_{i}}{\lambda_{i}}\right)^{n} d x_{i-1} \frac{d x_{i}}{\mu_{i}^{n}}=\frac{d x_{i-1} d x_{i}}{\lambda_{i}^{n}} \\
& f\left(x_{i-1}\right) \leq f(x)+\omega(f, \varepsilon)(x)=f_{\varepsilon}(x) \\
& \left|x_{i-1}-x_{i}\right|<\lambda_{i} \varepsilon .
\end{aligned}
$$

Applying these estimates (and also multiplying and dividing terms by $\lambda_{i}$ ), we see that (1.0v) implies (1.0iii).

The refinement estimate implies the existence of a limit measure because it gives a quantitative estimate of the sense in which the approximate energy 
functionals increase as $\varepsilon$ decreases. In particular, for fixed $\varepsilon$ we let $\varepsilon^{\prime}$ be small, and take each $\lambda_{i}=\frac{1}{\left[\varepsilon / \varepsilon^{\prime}\right]}$ (where [j denotes the greatest integer function) to deduce

$$
\tilde{E}_{\left[\varepsilon / \varepsilon^{\prime}\right] \varepsilon^{\prime}}(f) \leq \tilde{E}_{\varepsilon^{\prime}}\left(f_{\varepsilon}\right)
$$

as a special case of (1.0iii). We let $\varepsilon^{\prime} \rightarrow 0$ and note that the numbers $\tilde{E}_{\varepsilon}(f)$ vary continuously in $\varepsilon$ (because we used ball-average approximate energy densities), to conclude

$$
\tilde{E}_{\varepsilon}(f) \leq \liminf _{\varepsilon^{\prime} \rightarrow 0} \tilde{E}_{\varepsilon^{\prime}}\left(f_{\varepsilon}\right)
$$

Noting that

$$
\tilde{E}_{\varepsilon^{\prime}}\left(f_{\varepsilon}\right)=\tilde{E}_{\varepsilon^{\prime}}(f)+\tilde{E}_{\varepsilon^{\prime}}((\omega(f, \varepsilon)(x))
$$

and using the finite-energy hypothesis we see that

$$
\limsup _{\varepsilon \rightarrow 0} \tilde{E}_{\varepsilon}(f) \leq \liminf _{\varepsilon^{\prime} \rightarrow 0} \tilde{E}_{\varepsilon^{\prime}}(f) .
$$

This shows that the limit functional is well defined on non-negative functions $f \in C_{c}(\Omega)$. It is then an easy matter to show the limit functional is defined on all of $C_{c}(\Omega)$, and hence to deduce the existence of the limit Sobolev energy measure.

We carry out the general version of the above argument in $\S 1.2-1.5$. The lemmas are separated in such a way so that we may appeal to them again in $\S 1.7$, where we construct directional energy measures for Sobolev maps. In this case one fixes a Lipschitz vector field $Z$ and defines the $\varepsilon$-approximate energy density by

$$
z_{\varepsilon}(x)=\frac{d^{p}(u(x), u(\bar{x}(x, \varepsilon)))}{\varepsilon^{p}},
$$

where $\bar{x}(x, \varepsilon)$ is the point obtained by flowing time $\varepsilon$ along $Z$, starting at $x$. The approximate directional-energy measures also converge to a limit measure, and in $\S 1.8$ we derive some useful estimates and properties of the directional energies, in addition to proving that the Sobolev energy is an average of the directional energies.

In $§ 1.6$ we prove the lower semicontinuity of Sobolev energies: If

$$
\left\{u_{i}\right\} \subset W^{1, p}(\Omega, X) \quad\left(\text { or }\left\{u_{i}\right\} \subset B V(\Omega, X)\right) \text {, }
$$


is a sequence with uniformly bounded energies, and if $u_{i} \rightarrow u$ in $L^{p}(\Omega, X)$, then $u$ is a finite energy map and its energy measure $d e^{u}$ satisfies

$$
d e^{u} \leq \liminf _{i \rightarrow \infty} d e^{u_{i}} .
$$

In this section we also verify that for $X=\mathbb{R}$ our construction gives the usual Sobolev (and BV) spaces, and that the energy densities correspond in the expected way.

In $\S 1.9$ we develop the differentiability theory of directional energies. By restricting to integral curves of vector fields, one is able to reduce to understanding finite-energy maps from intervals to $X$, and so one can mimic classical differentiation theory. The final results (Theorem 1.9.6) are that for $p>1$ the directional energies are absolutely continuous with respect to Lebesgue measure, so can be written as

$$
\left|u_{*}(Z)\right|^{p} d \mu_{g}(x)
$$

for an $L^{p}$ function $\left|u_{*}(Z)\right|$, and that for suitable choices of $\nu$ the $\varepsilon$-approximate energies converge almost everywhere to $\left|u_{*}(Z)\right|^{p}$. It is then an easy matter in $\S 1.10$ to show that the Sobolev energy measure is also given by an $L^{1}$ density function, in case $p>1$.

In $\S 1.11$ we collect some estimates for the directional energy density functions which are needed in chapter 2. The $L^{p}$ trace theory for Sobolev maps from Lipschitz domains is developed in $\S 1.12$. Our approach is to use auxiliary transverse vector fields and to show that $u$ has well-defined limits along almost all of the corresponding integral curves, i.e. we follow the classical approach. In Theorem 1.12.2 we show that a sequence of bounded energy maps which converges in $L^{p}(\Omega, X)$ to a limit map $(p>1)$, has the property that the corresponding trace maps also converge, in $L^{p}(\partial \Omega, X)$. We also characterize maps $u, v$ with equal traces as those for which the real-valued functions $d(u, v)$ are Sobolev functions with trace zero. These facts are useful for the study of energy minimizing maps, in Section 2. Theorem 1.12.3 states that if $\Omega$ can be decomposed into Lipschitz subdomains, then finite energy maps with equal traces on the boundaries define in the aggregate a finite energy map on $\Omega$, with total energy given by the sum of the individual energies. This theorem 
will be used in the second chapter, where we use replacement techniques to study the equivariant harmonic map problem.

Finally, in $\S 1.13$, we include a natural precompactness theorem, which generalizes the fact that sequences in $W^{1, p}(\Omega, \mathbb{R})$ (or in $B V(\Omega, \mathbb{R})$ ) of uniformly bounded norm have convergent subsequences in $L^{p}(\Omega, \mathbb{R})$. We do not use this result in Section 2, but it is natural and follows quickly from the tools developed in here, so we include it.

1.1. Preliminary definitions. We will say that $(\Omega, g)$ is a Riemannian domain if it is a connected, open subset of a Riemannian manifold $(M, g)$, having the property that its metric completion $\bar{\Omega}$ is a compact subset of $M$. For $x, y \in \Omega$ we will denote the distance between $x$ and $y$ (on $(M, g)$ ) by $|x-y|$.

Define

$$
\Omega_{\varepsilon}=\{x \in \Omega \mid \operatorname{dist}(x, \partial \Omega)>\varepsilon\} .
$$

For $x \in \Omega, v \in T_{x} \Omega$, let $\exp (x, v)$ denote the (exponential) tangent map, i.e. $\exp (x, v)=\gamma(1)$, where $\gamma$ is the constant-speed geodesic satisfying $\gamma(0)=$ $x, \gamma^{\prime}(0)=v$.

If $Z$ is a Lipschitz vector field on $\bar{\Omega}$, we will write $Z \in \Gamma(T \bar{\Omega})$. In analogy to the exponential map, write $\bar{x}(x, t)$ for the flow induced by $Z$, i.e. $\bar{x}(x, t)=\gamma(t)$ where $\gamma$ solves

Write

$$
\left\{\begin{array}{l}
\frac{d}{d t} \gamma=Z(\gamma(t)) \\
\gamma(0)=x
\end{array}\right.
$$

$$
{ }^{z} \Omega_{\varepsilon} \equiv\left\{\left.x \in \Omega|\operatorname{dist}(x, \partial \Omega)>\varepsilon| Z\right|_{\infty}\right\} .
$$

If $(\Omega, g)$ is a Riemannian domain, if $(X, d)$ is a complete metric space, and if $1 \leq p<\infty$, then there is a natural definition of the space $L^{p}(\Omega, X)$. It is the set of Borel-measurable functions $u: \Omega \rightarrow X$ having separable range for which

$$
\int_{\Omega} d^{p}(u(x), Q) d \mu_{g}(x)<\infty
$$

for some $Q \in X$.

If $u$ and $v$ are two Borel-measurable functions with separable range in $X$, then the pair $(u(x), v(x))$ is a measurable function to $X \times X$. Thus $d^{p}(u(x), v(x)): \Omega \rightarrow X \times X \rightarrow \mathbb{R}$ is a measurable function. Thus integrals 
of $d^{p}(u(x), v(x))$ are well-defined, of which the one above is an example ([F] 2.3.2). It is straightforward to show that $L^{p}(\Omega, X)$ is a complete metric space, with distance function $\mathrm{D}$ defined by

$$
D^{p}(u, v)=\int_{\Omega} d^{p}(u(x), v(x)) d \mu_{g}(x) .
$$

The proof of this fact follows from the considerations in ([F] 2.4.12), but we quickly sketch the ideas. The triangle inequality for $d$, combined with that for real-valued $L^{p}$ functions implies that if $u$ is $L^{p}$, then

$$
\int_{\Omega} d^{p}(u(x), P) d \mu_{g}(x)<\infty
$$

for any $P \in X$. Another application of these two triangle inequalities shows that $D^{p}(u, v)$ is finite whenever $u, v \in L^{p}(\Omega, X)$. The triangle inequality for $D$ follows by the same argument. The proof that $L^{p}(\Omega, X)$ is complete mimics the usual proof for real-valued functions: Given a Cauchy sequence one finds a subsequence which converges a.e., shows the limit function is in $L^{p}(\Omega, X)$, and then that the sequence converges to the limit function in the $D$ metric.

1.2. Approximate energies. Fix $1 \leq p<\infty$ and $u \in L^{p}(\Omega, X)$. Let $V \in$ $\Gamma(T \bar{\Omega})$ be a smooth vector field on $\bar{\Omega}$. Then for $\varepsilon>0$ small the map $y=$ $\exp (x, \varepsilon V)$ is a diffeomorphism between ${ }^{V} \Omega_{\varepsilon}$ and its image, approaching the identity map as $\varepsilon \rightarrow 0$. Therefore the map $x \rightarrow u\left(\exp (x, \varepsilon V)\right.$ is in $L^{p}\left({ }^{V} \Omega_{\varepsilon}, X\right)$ and we have the estimate

$$
\left.\int_{\Omega_{\Omega_{\varepsilon}}} d^{p}(u(x), u(x), \exp (x, \varepsilon V))\right) d \mu_{g}(x) \leq C,
$$

independently of $\varepsilon$.

Now, for $(x, y) \in \Omega \times \Omega$ define

$$
e_{\varepsilon}(x, y)=\frac{d^{p}(u(x), u(y))}{\varepsilon^{p}} .
$$

For $x \in \Omega_{\varepsilon}$ define

$$
\begin{gathered}
S(x, \varepsilon)=\{y \text { s.t. }|y-x|=\varepsilon\} \\
d \sigma_{x, \varepsilon}(y)=(n-1)-\text { dimensional surface measure on } S(x, \varepsilon) .
\end{gathered}
$$


Finally, define the (spherically averaged) $\varepsilon$-approximate energy density function for $x \in \Omega_{\varepsilon}$, by

$$
e_{\varepsilon}(x)=\int_{S(x, \varepsilon)} e_{\varepsilon}(x, y) \frac{d \sigma_{x, \varepsilon}(y)}{\varepsilon^{n-1}}
$$

(Define $e_{\varepsilon}(x)$ to be zero otherwise .) We claim that $e_{\varepsilon}$ is a real-valued $L^{1}$ function, with

$$
\int_{\Omega_{\varepsilon}} e_{\varepsilon}(x) d \mu(x) \leq C \varepsilon^{-p} .
$$

To see this we reduce (by standard partition of unity arguments) to the case where $\bar{\Omega}$ has a global orthonormal frame $\left\{e_{1}, \ldots e_{n}\right\}$. Identify $\omega=\omega^{i} \partial_{i} \in$ $S^{n-1}(0,1) \subset \mathbb{R}^{n}$ with $\omega^{i} e_{i} \in S(0,1)_{x} \in T \Omega_{x}$. Then the map

$$
(x, \omega) \longrightarrow \exp (x, \varepsilon \omega) \longrightarrow u(\exp (x, \varepsilon \omega))
$$

is measurable and is easily seen to be in $L^{p}\left(\Omega_{\varepsilon} \times S(0,1)\right)$. In fact, by Tonelli's and Fubini's Theorems, and by applying the estimate (1.2i) we have

$$
\int_{\Omega_{\varepsilon}} \int_{S(0,1)} e_{\varepsilon}(x, \exp (x, \varepsilon \omega)) d \sigma(\omega) d \mu(x) \leq C \varepsilon^{-p}
$$

(for some other generic constant $C$ ). For $y=\exp (x, \varepsilon \omega)$ we note that

$$
\frac{d \sigma_{x, \varepsilon}(y)}{\varepsilon^{n-1} d \sigma(\omega)}
$$

is a continuous bounded function of $(x, \omega)$, independently of $\varepsilon$. Hence we may multiply the integrand above by this factor and maintain measurability. Furthermore we may deduce

$$
\int_{\Omega_{\varepsilon}} \int_{S(x, \varepsilon)} e_{\varepsilon}(x, y) \frac{d \sigma_{x, \varepsilon}(y)}{\varepsilon^{n-1}} d \mu(x) \leq C \varepsilon^{-p} .
$$

This verifies (1.2iii).

It is convenient to work with a variety of average energies. Let $\nu$ be any Borel measure on the interval $(0,2)$ satisfying

$$
\nu \geq 0, \quad \nu((0,2))=1, \quad \int_{0}^{2} \lambda^{-p} d \nu(\lambda)<\infty .
$$


Define the approximate energy density function ${ }_{\nu} e_{\varepsilon}(x)$ by averaging the spherical averages $e_{\varepsilon}(x)$ :

$$
{ }_{\nu} e_{\varepsilon}(x)=\int_{0}^{2} e_{\lambda \varepsilon}(x) d \nu(\lambda),
$$

for $x \in \Omega_{2 \varepsilon}$ (and ${ }_{\nu} e_{\varepsilon}(x)=0$ otherwise). It is easy to see that ${ }_{\nu} e_{\varepsilon}(x)$ is measurable, and from (1.2iii) and the integrability requirement in (1.2iv) we can estimate

$$
\int_{\Omega_{2 \varepsilon}}{ }_{\nu} e_{\varepsilon}(x) d \mu(x) \leq C \varepsilon^{-p} .
$$

A particular choice of $\nu$ which we will have occasion to use (besides the choice $\nu=\delta(1)$ which corresponds to our original energy density approximant) is the one which leads to uniform ball averaging, namely

$$
d \nu(\lambda)=(n+p) \lambda^{n+p-1} d \lambda \quad 0<\lambda<1 .
$$

For $u: \Omega \rightarrow \mathbb{R}$ smooth it is easy to see from our definitions that

$$
\begin{gathered}
\lim _{\varepsilon \rightarrow 0}{ }_{\nu} e_{\varepsilon}(x)=c_{n, p}|\nabla u(x)|^{p} \\
c_{n, p}=\int_{S^{n-1}}\left|x^{1}\right|^{p} d \sigma(x),
\end{gathered}
$$

(where $x=\left(x^{1}, \ldots x^{n}\right) \in \mathbb{R}^{n}$ and $S^{n-1}=\{|x|=1\}$ ). In particular, $c_{n, 2}=\omega_{n}$. If $u: \Omega \rightarrow N^{k}$ is a smooth map between Riemannian manifolds, then one can check that for $p=2, e_{\varepsilon}(x) \rightarrow \omega_{n}|\nabla u(x)|^{2}$ as well. For $p \neq 2$, however, $e_{\varepsilon}$ does not converge to a multiple of $|\nabla u(x)|^{p} \equiv\left(|\nabla u(x)|^{2}\right)^{p / 2}$, when $k>1$.

1.3. The functionals ${ }_{\nu} E_{\varepsilon}$. Let $1 \leq p<\infty, u \in L^{P}(\Omega, X)$, and $\nu$ as in (1.2iv). Then for $\varepsilon>0$ and $f \in C_{c}(\Omega)$ define

$$
{ }_{\nu} E_{\varepsilon}(f)=\int_{\Omega} f(x){ }_{\nu} e_{\varepsilon}(x) d \mu(x) .
$$

(In case $\nu=\delta(1)$ we suppress the $\nu$ in our notation.) We say that $u$ has finite energy (and write $u \in W^{1, p}(\Omega, X)$ for $p>1$ and $u \in B V(\Omega, X)$ for $p=1$ ), if 
for some $\nu$ as above

$$
\sup _{\substack{f \in C_{c}(\Omega) \\ 0 \leq f \leq 1}}\left(\limsup _{\varepsilon \rightarrow 0}{ }_{\nu} E_{\varepsilon}(f)\right) \equiv{ }_{\nu} E<\infty .
$$

(We show in Lemma 1.4.1 that the expression above is finite or infinite independently of $\nu$.) Let $\Omega$ be a locally compact metric space. For $f \in C_{c}(\Omega)$ define

$$
\begin{aligned}
|f| & =\max _{x \in \Omega}|f(x)| \\
\omega(f, \varepsilon)(x) & =\max _{|y-x| \leq \varepsilon}|f(y)-f(x)| \\
\omega(f, \varepsilon) & =\max _{x} \omega(f, \varepsilon)(x) .
\end{aligned}
$$

For $C>0$ define

$$
f_{\varepsilon}^{C}(x)=(1+C \varepsilon)(f(x)+\omega(f, 2 \varepsilon)(x))
$$

We now prove a basic "subpartitioning lemma" which will essentially guarantee the existence of energy-density measures for finite energy maps. It is the integral analog of the (trivial) lemma (for curves into metric spaces) that the approximate length of a curve with respect to a partition increases if the partition is refined. This monotone property is the essential ingredient in showing that rectifiable curves into metric spaces have well-defined length measures, and the lemma below will play the same role here.

Lemma 1.3.1. Let $1 \leq p<\infty, u \in L^{p}(\Omega, X)$, $\nu$ as in (1.2iv). Then for $f \in$ $C_{c}(\Omega), f \geq 0$, there exists a constant $C>0$ (depending only on Ricci curvature control of the metric $g$ ), so that the following "sub-partition estimate" holds for all sufficiently small $\varepsilon>0$ :

$$
{ }_{\nu} E_{\varepsilon}(f)^{1 / p} \leq \sum_{i} \lambda_{i}\left({ }_{\nu} E_{\lambda_{i} \varepsilon}\left(f_{\varepsilon}^{C}\right)\right)^{1 / p}
$$

Here

$$
\sum \lambda_{i}=1, \text { each } \lambda_{i}>0, \text { and the sum is a finite one. }
$$


Proof. We first consider the case $\nu=\delta(1)$, i.e. $e_{\varepsilon}$ in (1.2ii). For $\varepsilon>0$ small we may write $E_{\varepsilon}(f)$ more symmetrically as an integral over $\Omega \times \Omega$ :

$$
E_{\varepsilon}(f)=\iint_{|x-y|=\varepsilon} f(x) e_{\varepsilon}(x, y) d \sigma_{\varepsilon}(x, y)
$$

where $d \sigma_{\varepsilon}(x, y)$ is normalized $(2 n-1)$-dimensional surface measure on $\{\mid x-$ $y \mid=\varepsilon\}$, which can be expressed in terms of $d \sigma_{x, \varepsilon}(y)$ from (1.2ii):

$$
d \sigma_{\varepsilon}(x, y) \equiv \frac{d \sigma_{x, \varepsilon}(y)}{\varepsilon^{n-1}} d \mu(x)=\frac{d \sigma_{y, \varepsilon}(x)}{\varepsilon^{n-1}} d \mu(y) .
$$

Let $\left\{\lambda_{1} \ldots, \lambda_{\ell}\right\}$ satisfy (1.3vi). For any $x$ in the support of $f$, and for $\varepsilon$ sufficiently small, there is a unique geodesic from $x$ to any $y$ with $|x-y|=\varepsilon$. Let $\varphi:[0,1] \rightarrow \Omega$ be the (constant-speed) geodesic path from $x$ to $y$. Define the subdivision

$$
\begin{aligned}
x_{0} & =x \\
x_{i} & =\varphi\left(\sum_{k=1}^{i} \lambda_{k}\right), \quad i=1, \ldots, \ell .
\end{aligned}
$$

Then

$$
\left|x_{i}-x_{i-1}\right|=\lambda_{i} \varepsilon
$$

and the $X$-triangle inequality implies

$$
d(u(x), u(y)) \leq \sum_{i=1}^{n} d\left(u\left(x_{i-1}\right), u\left(x_{i}\right)\right) .
$$

The standard (iterated) $L^{p}$-triangle inequality (applied to $\left(f e_{\varepsilon}\right)^{1 / p}$ in (1.3vi)) yields

(1.3ix) $E_{\varepsilon}(f)^{1 / p} \leq \sum_{i=1}^{l} \lambda_{i}\left(\int_{\left|x_{i}-x_{i-1}\right|=\lambda_{i} \varepsilon} f(x) e_{\lambda_{i} \varepsilon}\left(x_{i-1}, x_{i}\right) d \sigma_{\varepsilon}(x, y)\right)^{1 / p}$.

For each fixed $i$ we may change variables from $(x, y)$ to $\left(x_{i-1}, x_{i}\right)$ in the corresponding term of the above inequality. It is easy to show that

$$
d \sigma_{\varepsilon}(x, y) \leq(1+C \varepsilon) d \sigma_{\lambda_{i} \varepsilon}\left(x_{i-1}, x_{i}\right)
$$

where the constant $C$ depends on Ricci curvature control of $g$. (If $\Omega$ is Euclidean, the last inequality is an equality with $C=0$.) We dominate $f(x)$ by 
$f\left(x_{i-1}\right)+\omega(f, \varepsilon)\left(x_{i-1}\right)$ in (1.3ix) and also use (1.3x) to deduce the sub-partition estimate (1.4i) in this case.

The proof for general $\nu$ is essentially the same. We may write

$$
{ }_{\nu} E_{\varepsilon}(f)=\int_{0}^{2} \int_{|x-y|<2 \varepsilon} f(x) e_{\varepsilon}(x, y) d \sigma_{\lambda \varepsilon}(x, y) \lambda^{-p} d \nu(\lambda) .
$$

One derives the general version of (1.3ix) by repeating the geodesic subdivision detailed above, this time for arbitrary $x \in \operatorname{supp}(f)$ with $|x-y|<2 \varepsilon$. The result is

${ }_{\nu} E_{\varepsilon}(f)^{1 / p} \leq \sum \lambda_{i}\left(\int_{0}^{2} \int_{\left|x_{i}-x_{i-1}\right|<2 \varepsilon \lambda_{i}} f(x) e_{\lambda_{i} \varepsilon}\left(x_{i-1}, x_{i}\right) d \sigma_{\lambda \varepsilon}(x, y) \lambda^{-p} d \nu(\lambda)\right)^{1 / p}$.

Noting that

$$
d \sigma_{\lambda \varepsilon}(x, y) \leq(1+C \varepsilon) d \sigma_{\lambda_{i} \lambda \varepsilon}\left(x_{i-1}, x_{i}\right),
$$

we may proceed as above to conclude (1.4i) in this general case.

1.4. Functional analysis lemmas. We prove two lemmas which enable us to conclude the existence of the energy-density measures for finite-energy maps. We will use these lemmas again when we discuss directional energies.

Lemma 1.4.1. Let $\Omega$ be a locally compact metric space. Let $\left\{\mathcal{L}_{\varepsilon}\right\}_{0<\varepsilon \leq \varepsilon_{0}}$ be a family of positive linear functionals on $C_{c}(\Omega)$. Let $1 \leq p<\infty$. Suppose there exists a $C>0$ so that for $f \in C_{c}(\Omega), f \geq 0$, for the function $f_{\varepsilon}^{C}$ defined in (1.3iv), and for any $\left\{\lambda_{i}\right\}$ satisfying (1.3vi) the subpartition inequality below holds when $\varepsilon>0$ is small:

$$
\left(\mathcal{L}_{\varepsilon}(f)\right)^{1 / p} \leq \sum_{i} \lambda_{i}\left(\mathcal{L}_{\lambda_{i} \varepsilon}\left(f_{\varepsilon}^{C}\right)\right)^{1 / p}
$$

Let $\nu$ be a non-negative Borel measure satisfying (1.2iv). Assume

$$
{ }_{\nu} \mathcal{L}_{\varepsilon}(f) \equiv \int_{0}^{2} \mathcal{L}_{\rho \varepsilon}(f) d \nu(\rho)
$$


is well-defined (i.e. $\mathcal{L}_{\varepsilon}(f)$ is Borel measurable in $\varepsilon$ for any $f \in C_{c}(\Omega)$ ). Assume

$$
\sup _{\substack{f \in C_{\mathrm{c}}(\Omega) \\ 0 \leq f \leq 1}}\left(\limsup _{\varepsilon \rightarrow 0}{ }_{\nu} \mathcal{L}_{\varepsilon}(f)\right) \equiv{ }_{\nu} L<\infty .
$$

Then also,

$$
\sup _{\substack{f \in C_{c}(\Omega) \\ 0 \leq f \leq 1}}\left(\limsup _{\varepsilon \rightarrow 0} \mathcal{L}_{\varepsilon}(f)\right) \equiv L<\infty .
$$

Proof. By hypothesis, if $f \geq 0, f \in C_{c}(\Omega)$ then for $\varepsilon>0$ small

$$
{ }_{\nu} \mathcal{L}_{\varepsilon}(f)=\int_{0}^{2} \mathcal{L}_{\rho \varepsilon}(f) d \nu(\rho) \leq C|f|
$$

where $C$ is independent of $f$. Hence

$$
\int_{0}^{1} \int_{0}^{2} \mathcal{L}_{\mu \rho \varepsilon}(f) d \nu(\rho) d \mu \leq C|f|
$$

i.e.

$$
\int_{0}^{2}\left(\int_{0}^{1} \mathcal{L}_{\mu \rho \varepsilon}(f) d \mu\right) d \nu(\rho) \leq C|f|
$$

Now, there is a $\delta>0$ so that $\nu((\delta, 2))>1 / 2$. Thus there is a $\rho \in(\delta, 2)$, with

$$
\int_{0}^{1} \mathcal{L}_{\mu \rho \varepsilon}(f) d \mu \leq 2 C|f|
$$

Writing $\varepsilon^{\prime}=\varepsilon \delta$, we have

$$
\int_{0}^{1} \mathcal{L}_{\mu(\rho / \delta) \varepsilon^{\prime}}(f) d \mu \leq 2 C|f| .
$$

Changing variables to $\mu^{\prime}=\mu(\rho / \delta)$, noting $1 \leq \rho / \delta \leq{ }^{2} / \delta$, yields

$$
\int_{0}^{1} \mathcal{L}_{\mu^{\prime} \varepsilon^{\prime}}(f) d \mu^{\prime} \leq \frac{4 C}{\delta}|f|
$$

for $f \in C_{c}(\Omega)$ and $\varepsilon^{\prime}$ small (depending on $\operatorname{supp}(f)$ ).

Now fix $f \in C_{c}(\Omega), 0 \leq f \leq 1$. We have from (1.4i)

$$
\mathcal{L}_{\varepsilon}(f) \leq 2^{p} \mathcal{L}_{\lambda \varepsilon}\left(f_{\varepsilon}^{C}\right)+2^{p} \mathcal{L}_{(1-\lambda) \varepsilon}\left(f_{\varepsilon}^{C}\right),
$$

for small $\varepsilon$. In particular,

$$
\mathcal{L}_{\varepsilon}(f) \leq 2^{p+1} \int_{0}^{1} \mathcal{L}_{\lambda \varepsilon}\left(f_{\varepsilon}^{C}\right) d \lambda
$$


For $\varepsilon$ small the functions $f_{\varepsilon}^{C}$ have uniformly-bounded supremum and uniform compact support. Hence we use (1.4iv) for the function $f_{\varepsilon}^{C}$ to estimate the integral in $(1.4 \mathrm{v})$, obtaining

$$
\mathcal{L}_{\varepsilon}(f) \leq C^{\prime}
$$

Lemma 1.4.2. Let $\Omega$ be a locally compact metric space. Let $\left\{\mathcal{L}_{\varepsilon}\right\}_{0<\varepsilon \leq \varepsilon_{0}}$ be a family of positive linear functionals on $C_{c}(\Omega)$. Let $1 \leq p<\infty$. Suppose there exists $a C>0$ so that for $f \in C_{c}(\Omega), f \geq 0$, for the function $f_{\varepsilon}^{C}$ defined in (1.3iii), and for any $\left\{\lambda_{i}\right\}$ satisfying (1.3vi) the subpartition inequality (1.4i) holds when $\varepsilon>0$ is small. Suppose also that the boundedness hypothesis (1.4iii) holds. Then

$$
\lim _{\varepsilon \rightarrow 0} \mathcal{L}_{\varepsilon}(f) \equiv \mathcal{L}(f)
$$

exists for any $f \in C_{c}(\Omega)$, and defines a positive linear functional $\mathcal{L}$ with $\|\mathcal{L}\|=$ L. Furthermore, we have the inequality

$$
\mathcal{L}_{\varepsilon}(f) \leq \mathcal{L}\left(f_{\varepsilon}^{C}\right)
$$

when $f \in C_{c}(\Omega), f \geq 0$, and $\varepsilon>0$ is sufficiently small.

Proof. Let $\varepsilon^{\prime} \ll \varepsilon$, and write [] for the greatest integer function. Let $\lambda_{i}=\left(\frac{\varepsilon^{\prime}}{\varepsilon}\right)$ for $i=1, \ldots k$, where $k=\left[\frac{\varepsilon}{\varepsilon^{\prime}}\right]$. Let $\lambda_{k+1}=1-\left(\frac{\varepsilon^{\prime}}{\varepsilon}\right) k$ if necessary. Applying (1.4i) and using (1.6i) for sufficiently small $\varepsilon$, we estimate

$$
\left(\mathcal{L}_{\varepsilon}(f)\right)^{1 / p} \leq\left[\frac{\varepsilon}{\varepsilon^{\prime}}\right]\left(\frac{\varepsilon^{\prime}}{\varepsilon}\right)\left(\mathcal{L}_{\varepsilon^{\prime}}\left(f_{\varepsilon}^{C}\right)\right)^{1 / p}+\left(\frac{\varepsilon^{\prime}}{\varepsilon}\right)\left((L+1)\left|f_{\varepsilon}^{C}\right|\right)^{1 / p} .
$$

Letting $\varepsilon^{\prime} \rightarrow 0$ we see

$$
\mathcal{L}_{\varepsilon}(f) \leq \liminf _{\varepsilon^{\prime} \rightarrow 0} \mathcal{L}_{\varepsilon^{\prime}}\left(f_{\varepsilon}^{C}\right) .
$$

Thus by the boundedness hypothesis (1.4iii) we see that

$$
\mathcal{L}_{\varepsilon}(f) \leq \liminf _{\varepsilon^{\prime} \rightarrow 0} \mathcal{L}_{\varepsilon^{\prime}}(f)+|L|(C \varepsilon|f|+(1+C \varepsilon) \omega(f, \varepsilon)),
$$

so that

$$
\limsup _{\varepsilon \rightarrow 0} \mathcal{L}_{\varepsilon}(f) \leq \liminf _{\varepsilon^{\prime} \rightarrow 0} \mathcal{L}_{\varepsilon}^{\prime}(f)
$$


Thus (1.4vi) holds for $f \geq 0$. The statement for arbitrary $f \in C_{c}(\Omega)$ follows. It is clear $\mathcal{L}$ is a positive linear functional with $\|\mathcal{L}\|=L$. The estimate (1.4vii) follows from (1.4viii), as $\varepsilon^{\prime} \rightarrow 0$.

Remark. 1.4.3. If the functionals $\mathcal{L}_{\varepsilon}(f)$ vary continuously in $\varepsilon$ for any $f \in$ $C_{c}(\Omega)$, then we may deduce (1.4ix) using only the subpartition estimate: (1.4i) immediately implies (for $\varepsilon^{\prime}<\varepsilon$ )

$$
\mathcal{L}_{\left[\frac{\varepsilon}{\varepsilon^{\prime}}\right] \varepsilon^{\prime}}(f) \leq \mathcal{L}_{\varepsilon^{\prime}}\left(f_{\varepsilon}^{C}\right)
$$

which gives (1.4ix) as $\varepsilon^{\prime} \rightarrow 0$. (Without the boundedness assumption (1.4iii), of course, both of these numbers may be infinite.) Note that if $\nu$ satisfies (1.2iv) and is absolutely continuous with respect to $\lambda$, then the functionals ${ }_{\nu} E_{\varepsilon}$ (1.3i) satisfy the continuity hypothesis. We will use of this fact when discussing directional energies.

\subsection{The energy-density measure.}

Theorem 1.5.1. Let $1 \leq p<\infty, u \in L^{p}(\Omega, X)$ have finite energy ${ }_{\nu_{1}} E$ with respect to some measure $\nu_{1}$ satisfying (1.2iv). Then it has finite energy with respect to all such $\nu$, and each measure ${ }_{\nu} e_{\varepsilon}(x) d \mu(x)$ converges weakly to the same "energy density" measure de, having total mass $\nu_{\nu_{1}} E$. Furthermore, the estimate (1.4vii) holds for $\mathcal{L}_{\varepsilon}={ }_{\nu} E_{\varepsilon}$ and $\mathcal{L}=E$.

Proof. By Lemma 1.4.1 $u$ has finite energy with respect to the standard measure, $\nu=\delta(1)$. Hence its energy is finite with respect to any $\nu$ satisfying (1.2iv). From Lemmas 1.3.1,1.4.1,1.4.2, and the Riesz representation theorem for continuous linear functionals on $C_{c}(\Omega)$ we deduce that each ${ }_{\nu} e_{\varepsilon}(x) d \mu(x)$ converges weakly to a limit measure. The definition $(1.2 \mathrm{v})$ of ${ }_{\nu} E_{\varepsilon}$ shows that the limit measure is the one arising from $\nu=\delta(1)$, i.e the weak limit of $e_{\varepsilon}(x) d \mu(x)$. The fact that (1.4vii) holds as indicated is clear.

Remark. 1.5.2. For $1<p<\infty, u \in W^{1, p}(\Omega, X)$, it is easy to see that $u \in$ $W^{1, p^{\prime}}(\Omega, X)$, for any $1 \leq p^{\prime}<p$, and that the $p^{\prime}$-energy density measure $d e_{p^{\prime}}$ is absolutely continuous with respect to Lebesgue measure. (We will say that $u \in$ $W^{1,1}(\Omega, X)$ if $u \in B V(\Omega, X)$ and its energy density de is absolutely continuous with respect to Lebesgue measure.) In fact, if $E$ is the total (Sobolev) $p$-energy 
of $u$, then there is a constant $C$ (depending only on dimension $n$ ) so that for any Borel measurable set $S \subset \Omega$,

$$
e_{p^{\prime}}(S) \leq C\left(\mu_{g}(S)\right)^{\frac{p-p^{\prime}}{p}} E^{\frac{p^{\prime}}{p}}
$$

To see why this is true, it suffices to consider the case $S \subset \subset \Omega$. In this case, for any $\delta>0$ we may pick $f \in C_{c}(\Omega), 0 \leq f \leq 1$, with $f \equiv 1$ on $S$ and $\mu_{g}\left(\operatorname{supp}(f) \leq \mu_{g}(S)+\delta\right.$. Then by Hölder

$$
\begin{aligned}
E_{\varepsilon, p^{\prime}}(f) & =\int_{\Omega} f e_{\varepsilon, p^{\prime}}(x) d \mu(x) \\
& \leq C \int_{\Omega}\left(f^{\frac{p}{p^{\prime}}} e_{\varepsilon, p}(x) d \mu(x)\right)^{\frac{p^{\prime}}{p}}(\mu(\operatorname{supp}(f)))^{\frac{p-p^{\prime}}{p}} .
\end{aligned}
$$

Here we have used $E_{\varepsilon, p}(f)$ and $e_{\varepsilon, p}(x)$ to denote the approximate energy functional and $\varepsilon$-approximate energy function, corresponding to the power $p$. As $\varepsilon \rightarrow 0$ we conclude

$$
e_{p^{\prime}}(S) \leq E_{p^{\prime}}(f) \leq C E^{\frac{p^{\prime}}{p}}(\mu(S)+\delta)^{\frac{p-p^{\prime}}{p}}
$$

which shows our claim as $\delta \rightarrow 0$.

1.6. Lower semicontinuity, and consistency when $X=\mathbb{R}$.

Theorem 1.6.1. Let $1 \leq p<\infty,\left\{u_{k}\right\} \subset W^{1, p}(\Omega, X)$ if $p>1$ (or $\left\{u_{k}\right\} \subset$ $B V(\Omega, X)$ if $p=1)$. Let $u_{k} \rightarrow u$ in the $L^{p}$ metric. Write $e^{k}$ for the energydensity measure of $u_{k}, E^{k} \equiv e^{k}(\Omega)$. Assume there exists $E<\infty$ with each $E^{k}<E$. Then $u \in W^{1, p}(\Omega, X)$ if $p>1$ (or $u \in B V(\Omega, X)$ if $p=1$ ), and its energy-density measure e satisfies

$$
d e \leq \liminf d e^{k}
$$

as measures.

Proof. For $d \nu(\lambda)=(n+p) \lambda^{n+p-1} d \lambda$ (ball averages (1.2vii)) and for fixed $f \in C_{c}(\Omega)$ we have as a consequence of Lemma 1.3.1, (1.4vii) of Lemma 1.4.2, and Theorem 1.5.1, that

$$
{ }_{\nu} E_{\varepsilon}^{k}(f) \leq E^{k}\left(f_{\varepsilon}^{C}\right)=E^{k}(f)+E^{k}\left(f_{\varepsilon}^{C}-f\right) .
$$


Because we are using ball averages the energy-density functions ${ }_{\nu} e_{\varepsilon}^{k}(x)$ converge uniformly to ${ }_{\nu} e_{\varepsilon}(x)$ as $k \rightarrow \infty$ (and $\varepsilon>0$ is fixed). Thus

$$
\lim _{k \rightarrow \infty}{ }_{\nu} E_{\varepsilon}^{k}(f)={ }_{\nu} E_{\varepsilon}(f)
$$

Hence

$$
{ }_{\nu} E_{\varepsilon}(f) \leq \liminf _{k \rightarrow \infty} E^{k}(f)+E(C \varepsilon|f|+\omega(f, 2 \varepsilon)) .
$$

Thus $u$ has finite energy and if we let $\varepsilon \rightarrow 0$ we also deduce that

$$
E(f) \leq \liminf _{k \rightarrow \infty} E^{k}(f) .
$$

Theorem 1.6.2.

$$
\begin{aligned}
W^{1, p}(\Omega, \mathbb{R}) & =W^{1, p}(\Omega) \quad \text { for } \quad p>1 \\
B V(\Omega, \mathbb{R}) & =B V(\Omega) .
\end{aligned}
$$

Furthermore, the energy densities are constant multiples of each other in all cases.

Proof. We first assume $(\Omega, g)$ is Euclidean. If $u \in L^{p}(\Omega, \mathbb{R})$ is smooth, then $e_{\varepsilon}(x) \rightarrow c_{n, p}|\nabla u(x)|^{p}$ uniformly on compact subsets of $\Omega$, so it is clear that the measure de equals $c_{n, p}|\nabla u(x)|^{p} d \mu_{x}$. If $u \in L^{p}(\Omega, \mathbb{R})$ then we mollify it with a $C^{\infty}$ approximate identity:

$$
\begin{aligned}
\eta_{t}(x) & =t^{-\eta} \eta\left(\frac{x}{t}\right) \\
\eta(x) & \in C_{0}^{\infty}(B(0,1)), \quad \eta \geq 0, \quad \int_{B(0,1)} \eta=1 \\
\eta(x) & =\varphi(|x|) .
\end{aligned}
$$

We write $u_{t}=u * \eta_{t}$.

It is well-known that if $u \in W^{1, p}(\Omega)$ then $u_{t} \rightarrow u$ in $W_{\text {loc }}^{1, p}(\Omega)$, i.e. on any compact subset the convergence is in the $W^{1, p}$-norm [GT]. In particular, for $p>1$ we have that $\left|\nabla u_{t}\right|^{p} d \mu-|\nabla u|^{p} d \mu$ (weakly) as measures. By (the usual) Sobolev-Space semicontinuity, it is also true that $\int_{\Omega_{t}}\left|\nabla u_{t}\right|^{p} d \mu \rightarrow \infty$ in case $u \in L^{p}(\Omega)$ but $u \notin W^{1, p}(\Omega)$. In case $p=1$ it is true that $u \in B V(\Omega)$ implies $\left|\nabla u_{t}\right| d \mu$ converges weakly to the $B V$-measure $|\nabla u|$, and that $u \notin B V(\Omega)$ implies $\left.\int_{\Omega_{t}}\left|\nabla u_{t}\right| d \mu \rightarrow \infty\right)[\mathrm{Gi}]$. Thus Theorem 1.6.2 follows if we can show the corresponding statements for our energy densities. 
If $u \in L^{p}(\Omega, \mathbb{R})$ and $u$ does not have finite energy (in our sense) then it must follow that $\int_{\Omega_{t}}\left|\nabla u_{t}\right|^{p} d \mu \rightarrow \infty$, since otherwise lower semicontinuity (1.6i) would be violated by choosing an appropriate subsequence. Thus we need only show that if $u$ is a finite-energy map, then $c_{n, p}\left|\nabla u_{t}\right|^{p} d \mu=d e^{u_{t}} \rightarrow d e$. From our comments above, and from Theorem 1.6.1, we know that

$$
d e^{u} \leq \liminf _{t \rightarrow 0} d e^{u_{t}}
$$

so we only need the reverse inequality. We use $d \nu(\lambda)=(n+p) \lambda^{n+p-1} d \lambda$, i.e. ball averages, for the approximate energies and we take $f \in C_{c}(\Omega), f \geq 0$. Then

$$
\begin{aligned}
{ }_{\nu} E_{\varepsilon}^{u_{t}}(f) & =\int_{\Omega} f(x)_{\nu} e_{\varepsilon}^{u_{t}}(x) d x \\
& =(n+p) \int_{\Omega} \int_{B(0,1)} f(x) \frac{\left|u^{t}(x)-u^{t}(x+\varepsilon v)\right|^{p}}{\varepsilon^{p}} d v d x .
\end{aligned}
$$

By properties of convolution and Jensen's inequality

$$
\begin{aligned}
\left|u^{t}(x)-u^{t}(x+\varepsilon v)\right|^{p} & \leq|u(\cdot)-u(\cdot+\varepsilon v)|^{p} * \eta_{t} \\
& =\int_{B(0,1)}|u(x-t w)-u(x-t w+\varepsilon v)|^{p} \eta(w) d w .
\end{aligned}
$$

Hence

${ }_{\nu} E_{\varepsilon}^{u_{t}}(f) \leq \frac{n+p}{\varepsilon^{p}} \int_{B(0,1)} \int_{B(0,1)} \int_{\Omega} f(x) \eta(w)|u(x-t w)-u(x-t w+\varepsilon v)|^{p} d x d v d w$.

Letting $z=x-t w$, estimating $|f(x)-f(z)| \leq \omega(f, t)$, yields

$$
{ }_{\nu} E_{\varepsilon}^{u_{t}}(f) \leq \frac{n+p}{\varepsilon^{p}} \iiint f(z) \eta(w)|u(z)-u(z+\varepsilon v)|^{p} d z d v d w+C \omega(f, t),
$$

where $C$ depends on the energy of $u$ (for $\varepsilon$ small). That is

$$
{ }_{\nu} E_{\varepsilon}^{u_{t}}(f) \leq{ }_{\nu} E_{\varepsilon}^{u}(f)+C \omega(f, t)
$$

so $(\varepsilon \rightarrow 0)$,

$$
E^{u_{t}}(f) \leq E^{u}(f)+C \omega(f, t) .
$$

Hence

$$
\limsup _{t \rightarrow 0} E^{u_{t}}(f) \leq E^{u}(f)
$$


i.e.

$$
\limsup _{t \rightarrow 0} d e^{u_{t}} \leq d e^{u}
$$

In case $(\Omega, g)$ is a general Riemannian domain, we proceed as above and eventually are left to verify (1.6iv), which we do as follows. Express $\Omega$ as a finite union of open subsets, each of which have approximately Euclidean local coordinate charts. Use a subordinate partition of unity $\left\{\eta_{i}\right\}$ to express $E(f)$ as a sum of $E\left(\eta_{i}(f)\right)$. One may then work as above to estimate ${ }_{\nu} E_{\varepsilon}^{u_{t}}\left(\eta_{i} f\right)$, introducing a small error term into (1.6iv) from the fact that the metric isn't exactly Euclidean. This error term approaches zero as one takes subsets with diameter approaching zero. Thus one can verify (1.6iv) in the general case as well.

Corollary 1.6.3. Let $1 \leq p<\infty, u, v \in W^{1, p}(\Omega, X)$ (or $u, v \in B V(\Omega, X)$ if $p=1)$. Define the $p$-distance on $X \times X$, by

$$
d^{p}\left(\left(x_{1}, x_{2}\right),\left(y_{1}, y_{2}\right)\right)=d^{p}\left(x_{1}, y_{1}\right)+d^{p}\left(x_{2}, y_{2}\right)
$$

Let $f: X \times X \rightarrow \mathbb{R}$ be Lipschitz continuous, with Lipschitz constant $L$ (with respect to $D$ ). Then the map $h(x)=f\left(u(x), v(x)\right.$ ) satisfies $h \in W^{1, p}(\Omega)$ (or $h \in B V(\Omega)$ if $p=1)$, and

$$
c_{n, p}|\nabla h|^{p} d \mu \leq L^{p}\left(d e^{u}+d e^{v}\right)
$$

as measures.

Proof. The $\varepsilon$-energy density for $h$ is an average (with respect to $y$ ) of

$$
\begin{aligned}
\frac{|h(x)-h(y)|^{p}}{\varepsilon^{p}} & =\frac{|f(u(x), v(x))-f(u(y), v(y))|^{p}}{\varepsilon^{p}} \\
& \leq L^{p}\left(\frac{d^{p}(u(x), u(y))+d^{p}(v(x), v(y))}{\varepsilon^{p}}\right) .
\end{aligned}
$$

The result follows immediately. A special case (which is easy to check directly) is $f(p, q)=d(p, q)$, whence if $u, v \in W^{1, p}(\Omega, X)(\operatorname{resp} . B V(\Omega, X))$ we have $d(u, v) \in W^{1, p}(\Omega, \mathbb{R})($ resp. $B V(\Omega))$, and

$$
c_{n, p}|\nabla d(u, v)|^{p} d \mu \leq 2^{p}\left(d e^{u}+d e^{v}\right)
$$


If $v \equiv P$, we can argue directly as in (1.6v) and remove the factor of $2^{p}$ to get

$$
c_{n, p}|\nabla d(u, P)|^{p} d \mu \leq d e^{u} .
$$

1.7. Directional energies. It is natural to define directional energies for maps $u \in L^{p}(\Omega, X)$, that is, measures of their rate of change in directions of smooth vector fields. For finite-energy maps $\left(u \in W^{1, p}(\Omega)\right.$ or $u \in B V(\Omega)$ ) it will turn out that these directional energy densities are always well-defined measures, and the resulting calculus will be of use not only in this chapter, but in the later sections of the paper as well. Let $1 \leq p<\infty, u \in L^{p}(\Omega, X)$. Let $Z$ be a Lipschitz vector field on $\bar{\Omega}, Z \in \Gamma(T \bar{\Omega})$, and recall the definitions $\bar{x}(x, t)$ and ${ }^{z} \Omega_{\varepsilon}$ from 1.1. For $x \in{ }^{z} \Omega_{\varepsilon}$ define the $\varepsilon$ energy-density function (of $u$ in the direction $Z$ ) by

$$
{ }^{z} e_{\varepsilon}(x)=\frac{d^{p}(u(x), u(\bar{x}(x, \varepsilon)))}{\varepsilon^{p}} .
$$

(For $x \notin{ }^{Z} \Omega_{\varepsilon}$ define ${ }^{z} e_{\varepsilon}(x)=0$.) As in 1.1 we deduce ${ }^{z} e_{\varepsilon} \in L^{1}(\Omega, \mathbb{R})$, with

$$
\int_{\Omega}{ }^{z} e_{\varepsilon}(x) d \mu(x) \leq C \varepsilon^{-p}
$$

For a non-negative Borel measure $\nu$ satisfying (1.2iv) we write (suppressing the $Z$-dependence here and below)

$$
{ }_{\nu} e_{\varepsilon}(x)=\int_{(0,2)} e_{\rho \varepsilon}(x) d \nu(\rho)
$$

for $x \in{ }^{Z} \Omega_{2 \varepsilon}\left({ }_{\nu} e_{\varepsilon}(x)=0\right.$ otherwise). Clearly ${ }_{\nu} e_{\varepsilon} \in L^{1}(\Omega, \mathbb{R})$ as well. For $f \in C_{c}(\Omega)$ write

$$
\begin{aligned}
{ }_{\nu} E_{\varepsilon}(f) & =\int_{\Omega} f(x){ }_{\nu} e_{\varepsilon}(x) d \mu(x)=\int_{(0,2)}{ }_{\nu} E_{\rho \varepsilon}(f) d \nu(\rho), \\
{ }_{\nu} E & =\sup _{\substack{0 \leq f \leq 1 \\
f \in C_{c}(\Omega)}} \limsup _{\varepsilon \rightarrow 0} E_{\nu} E_{\varepsilon}(f) .
\end{aligned}
$$

In case some ${ }_{\nu} E<\infty$ we say $u$ has finite $(p-$ ) energy (in the direction $Z$ ). The analog of Theorem 1.7 for Sobolev (or BV) energies is the following result for directional energies: 
Theorem 1.7. Let $1 \leq p<\infty, u \in L^{p}(\Omega, X), Z$ as above. If for some $\nu_{1}$ as above we have ${ }_{\nu_{1}} E<\infty$, then ${ }_{\nu} E<\infty$ for all such $\nu$. For any such $\nu$ the measures ${ }_{\nu} e_{\varepsilon}(x) d \mu(x)$ converge weakly to an energy density de as $\varepsilon \rightarrow 0$, which is independent of $\nu$ and has total mass ${ }_{\nu_{1}} E$.

Proof. By scaling the domain metric we may assume $|Z| \leq 1$. If we can verify the "subpartition estimate" $(1.4 \mathrm{i})$ for $\mathcal{L}_{\varepsilon}(f) \equiv{ }_{\nu} E_{\varepsilon}(f)$, then Lemmas $1.3 .1,1.4 .1,1.4 .2$, and the Riesz representation theorem immediately imply our result, just as they yielded Theorem 1.5.1 Let $\left\{\lambda_{i}\right\}$ satisfy (1.3vi). Given $x, \bar{x}(x, \varepsilon)$, define $\bar{x}_{0}(x, \varepsilon)=x$ and

$$
\bar{x}_{i}(x, \varepsilon)=\bar{x}\left(x,\left(\sum_{j=1}^{i} \lambda_{j}\right) \varepsilon\right), \quad i \geq 1 .
$$

Now

$$
{ }_{\nu} E_{\varepsilon}(f)=\int_{\Omega} \int_{0}^{2} f(x) \frac{d^{p}(u(x), u(\bar{x}(x, \varepsilon \rho))}{\varepsilon^{p}} \rho^{-p} d \nu(\rho) d \mu(x) .
$$

Using triangle inequalities as in (1.3ix), we estimate

$$
\begin{aligned}
& \left({ }_{\nu} E_{\varepsilon}(f)\right)^{1 / p} \\
& \quad \leq \sum_{i} \lambda_{i}\left(\iint f(x) \frac{d^{p}\left(u\left(\bar{x}_{i-1}(x, \varepsilon \rho)\right), u\left(\bar{x}_{i}(x, \varepsilon \rho)\right)\right)}{\varepsilon^{p} \lambda_{i}^{p}} \rho^{-p} d \nu(\rho) d \mu(x)\right)^{1 / p} .
\end{aligned}
$$

For fixed $i$ (and $\rho$ ) we change variables from $x$ to $w=\bar{x}_{i-1}(x, \varepsilon \rho)$. Note that $\bar{x}_{i}(x, \varepsilon \rho)=\bar{x}\left(w, \varepsilon \rho \lambda_{i}\right)$, and estimate $f(x)$ above by $f(w)+\omega(f, 2 \varepsilon)(w), d \mu(x) \leq$ $(1+C \varepsilon) d \mu(w)$. (In this case $\mathrm{C}$ depends not only on the metric $g$ but also on the Lipschitz constant of $Z$.) Hence

$$
\begin{aligned}
\left({ }_{\nu} E_{\varepsilon}(f)\right)^{1 / p} & \leq \sum \lambda_{i}\left(\iint f_{\varepsilon}^{C}(w) \frac{d^{p}\left(u(w), u\left(\bar{x}\left(w, \varepsilon \rho \lambda_{i}\right)\right)\right)}{\left(\varepsilon \lambda_{i}\right)^{p}} \rho^{-p} d \nu(\rho) d \mu(x)\right)^{1 / p} \\
& =\sum \lambda_{i}\left({ }_{\nu} E_{\lambda_{i} \varepsilon}\left(f_{\varepsilon}^{C}\right)\right)^{1 / p} \cdot \square
\end{aligned}
$$

1.8. The calculus of directional energies. We collect some useful properties of the directional-derivative measures. We fix $1 \leq p<\infty, u \in W^{1, p}(\Omega, X)$ if $p>1$ or $u \in B V(\Omega, X)$ if $p=1$. We use $E(f)$ to denote the (Sobolev or BV) functional induced by $u$, writing $E$ for its norm. For $Z \in \Gamma(T \bar{\Omega})$ we write ${ }^{z} E(f)$ and ${ }^{z} E$ for the directional functional and its norm. 
Theorem 1.8.1. If $Z \in \Gamma(T \bar{\Omega})$ then ${ }^{Z} E<\infty$ and there is a constant $C$ (depending only on the dimension $n$ of $\Omega$ ) so that we can estimate

$$
d\left({ }^{Z} e\right) \leq C|Z|_{\infty}^{p} d e
$$

If $z(x, t)$ is any one-parameter family of $C^{1,1}$ diffeomorphisms, having Lipschitz velocity fields and satisfying

$$
z(x, 0)=x,\left.\quad \frac{\partial}{\partial t} z(x, t)\right|_{t=0}=Z(x)(x \in \Omega)
$$

for $Z \in \Gamma(T \bar{\Omega})$, then

$$
\frac{d^{p}(u(x), u(z(x, \varepsilon)))}{\varepsilon^{p}} \rightarrow d\left({ }^{Z} e\right)
$$

as $\varepsilon \rightarrow 0$.

For $p=1$ and $W \in \Gamma(T \bar{\Omega})$ we have the triangle inequality

$$
d\left({ }^{Z+W} e\right) \leq d\left({ }^{Z} e\right)+d\left({ }^{W} e\right)
$$

More generally, for any $1 \leq p<\infty$ and $f \in C_{c}(\Omega)$ with $f \geq 0$ we have

$$
\left({ }^{Z+W} E(f)\right)^{\frac{1}{p}} \leq\left({ }^{Z} E(f)\right)^{\frac{1}{p}}+\left({ }^{W} E(f)\right)^{\frac{1}{p}} .
$$

If $h$ is a Lipschitz function on $\bar{\Omega}$, then

$$
d\left({ }^{h Z} e\right)=|h|^{p} d\left({ }^{Z} e\right) .
$$

In case $(\Omega, g)$ has an orthonormal frame $\left\{e_{1}, \ldots, e_{n}\right\}$ we identify $S^{n-1} \subset \mathbb{R}^{n}$ with $S_{x}^{n-1} \subset T \Omega_{x}$ by

$$
\omega=\left(\omega^{1}, \ldots, \omega^{n}\right) \longmapsto \omega^{i} e_{i},
$$

and denote $\omega^{i} e_{i} \in \Gamma(T \Omega)$ by $\omega$ as well. Then we have

$$
E(f)=\int_{S^{n-1}}{ }^{\omega} E(f) d \sigma(\omega)
$$

Proof. These results are consequences of the following technical estimate:

Lemma 1.8.2. Let $\psi: \Omega \rightarrow \psi(\Omega) \subset M$ be a bi-Lipschitz map from $\Omega$ to its image. We think of $\psi$ being close to the identity map, and write $|\psi-i d|_{\infty}=\delta$. Assume $\delta$ is small enough so that the geodesic subdivision techniques of 1.4 are applicable whenever $x \in \Omega_{3 \delta}$ and $|x-y|<3 \delta$. Let $f \in C_{c}\left(\Omega_{3 \delta},\right), f \geq 0$. 
Then there exists a constant $C$ depending only on $n, p$, on the curvature of $(\Omega, g)$ and on first-derivative bounds for $\psi$ and $\psi^{-1}$, so that

$$
\int_{\Omega} f(x) d^{p}(u(x), u(\psi(x))) d \mu_{g}(x) \leq C \delta^{p} E|f|_{\infty} .
$$

Proof. For $x \in \Omega_{3 \delta}$ we write $\tilde{x}$ for the geodesic midpoint of $x$ and $\psi(x)$. Then from the triangle inequality and an average of integration over $B\left(\tilde{x}, \frac{\delta}{2}\right)$ we have

$$
\begin{aligned}
& \left.d^{p}(u(x), u(\psi(x))) \leq 2^{p}\left(\oint_{B\left(\tilde{x}, \frac{\delta}{2}\right)} d^{p}(u(x), u(y)) d \mu(y)+\oint_{B\left(\tilde{x}, \frac{\delta}{2}\right)} d^{p}(u(\psi(x)), u(y))\right) d \mu(y)\right) \\
& \leq C\left({ }_{\nu} e_{\delta}(x)+{ }_{\nu} e_{\delta}(\psi(x))\right) \delta^{p} .
\end{aligned}
$$

Here we are using $\nu$ corresponding to ball averages (1.2vii), and $\mathrm{C}$ depends on allowed quantities. We apply this estimate to the integrand in (1.8vii), change variables from $x$ to $\psi(x)$ in the second term, and deduce

$(1.8 \mathrm{viii}) \int_{\Omega} f(x) d^{p}(u(x), u(\psi(x))) d \mu(x) \leq C \delta^{p}\left({ }_{\nu} E_{\delta}(f)+{ }_{\nu} E_{\delta}(\tilde{f})\right)$,

where

$$
\tilde{f}(x)=f(x)+\omega(f, \delta)(x) .
$$

From (1.4vii) we see that this last estimate is bounded above by

$$
C \delta^{p}\left(E\left(f_{\delta}^{C}\right)+E\left((\tilde{f})_{\delta}^{C}\right)\right) \leq C \delta^{p} E|f|_{\infty} .
$$

This proves (1.8vii).

Our first claim (1.8.i) follows immediately from the reasoning above: We take $\psi(x)=\bar{x}(x, \varepsilon)$ and deduce from (1.8vii) that ${ }^{Z} E<\infty$. Then we take $\delta=\varepsilon|Z|_{\infty}$ in (1.8viii), divide by $\varepsilon^{p}$ and let $\varepsilon \rightarrow 0$, to conclude (1.8.i).

We show (1.8ii). From triangle inequalities we may estimate

$$
\begin{aligned}
& \left|\left(\int f(x) \frac{d^{p}(u(x), u(z(x, \varepsilon)))}{\varepsilon^{p}} d \mu(x)\right)^{\frac{1}{p}}-\left(\int f(x) \frac{d^{p}(u(x), u(\bar{x}(x, \varepsilon)))}{\varepsilon^{p}} d \mu(x)\right)^{\frac{1}{p}}\right| \\
& \leq\left(\int f(x) \frac{d^{p}(u(z(x, \varepsilon)), u(\bar{x}(x, \varepsilon)))}{\varepsilon^{p}} d \mu(x)\right)^{\frac{1}{p}} .
\end{aligned}
$$


For small $\varepsilon>0$ we consider $\psi$ which maps $z(x, \varepsilon)$ to $\bar{x}(x, \varepsilon)$. Since $|\psi-i d|_{\infty}$ is $o(\varepsilon)$ we deduce from (1.8vii) that the right hand side of the above inequality approaches zero as $\varepsilon \rightarrow 0$. This proves (1.8ii).

We next show (1.8iv), which yields (1.8iii) as a special case. We may assume we are working in a local coordinate chart. In this case we write

$$
\begin{aligned}
& \left(\int f(x) \frac{d^{p}(u(x), u(x+\varepsilon(Z+W)(x)))}{\varepsilon^{p}} d \mu(x)\right)^{\frac{1}{p}} \\
\leq & \left(\int f(x) \frac{d^{p}(u(x), u(x+\varepsilon Z(x)))}{\varepsilon^{p}} d \mu(x)\right)^{\frac{1}{p}} \\
+ & \left(\int f(x) \frac{d^{p}(u(y), u(y+\varepsilon W(x)))}{\varepsilon^{p}} d \mu(x)\right)^{\frac{1}{p}}
\end{aligned}
$$

where we have written $y$ for $x+\varepsilon Z(x)$ in the bottom integral. By (1.8ii) the top integral converges to ${ }^{Z+W} E(f)$ as $\varepsilon \rightarrow 0$, and the middle integral converges to ${ }^{Z} E(f)$. In fact, the bottom one converges to ${ }^{W} E(f)$, as one can deduce by changing variables from $\mathrm{x}$ to $\mathrm{y}$, dominating $f(x)$ by $f(y)+\omega\left(f, \varepsilon|W|_{\infty}\right)(y)$, and applying (1.8ii) again. This shows (1.8iv).

We next show $(1.8 \mathrm{v})$. We first consider the case of constant $h$ : If $h>0$ then we observe that $h^{p}\left({ }^{Z} e_{\varepsilon h}(x)\right)={ }^{h Z} e_{\varepsilon}(x)$. This implies the result as $\varepsilon \rightarrow 0$. In case $h=-1$, we note that ${ }^{-Z} e_{\varepsilon}(\bar{x}(x, \varepsilon))={ }^{Z} e_{\varepsilon}(x)$, so by the usual change of variables arguments we obtain $d\left({ }^{-Z} e\right)=d\left({ }^{Z} e\right)$. Thus $(1.8 \mathrm{v})$ holds when $h$ is any constant.

Next we assume that there is a constant $h_{0}$ and a small number $\delta>0$ so that $\left|h-h_{0}\right| \leq \delta$. We apply the triangle inequality (1.8iv) twice, with vector fields $h Z,\left(h_{0}-h\right) Z$ the first time and $h_{0} Z,\left(h-h_{0}\right) Z$ the second time. Taking $p^{\text {th }}$-powers, applying (1.8i), using the result for constant multiples which we just showed, and using standard inequalities, we see that for any $\varepsilon>0$,

$$
\begin{aligned}
& { }^{Z} E\left(h_{0}^{p} f\right) \leq(1+\varepsilon)^{p}\left({ }^{h Z} E(f)\right)+\left(\frac{1+\varepsilon}{\varepsilon}\right)^{p} C \delta^{p} E(f) \\
& { }^{h Z} E(f) \leq(1+\varepsilon)^{p}\left({ }^{Z} E\left(h_{0}^{p} f\right)\right)+\left(\frac{1+\varepsilon}{\varepsilon}\right){ }^{p} C \delta^{p} E(f) .
\end{aligned}
$$

These inequalities imply the measure inequalities

$$
\left|h_{0}\right|^{p} d\left({ }^{Z} e\right)-c(\varepsilon, \delta) d e \leq d\left({ }^{h Z} e\right) \leq\left|h_{0}\right|^{p} d\left({ }^{Z} e\right)+c(\varepsilon, \delta) d e
$$


where $c(\varepsilon, \delta)$ has the property that it can be made arbitrarily small by choosing $\varepsilon$ (and then) $\delta$ small. We can now show the general case of $(1.8 \mathrm{v})$. Pick $c(\varepsilon, \delta)$ small. Cover $\Omega$ with a finite number of open subsets $\left\{U_{i}\right\}$ so that there are constants $\left\{h_{i}\right\}$ with $\left|h-h_{i}\right|<\delta$ on $U_{i}$. Pick a subordinate partition of unity $\left\{\eta_{i}\right\}$ to the $\left\{U_{i}\right\}$. Note that ${ }^{h Z} E(f)=\sum_{i}{ }^{h Z} E\left(\eta_{i} f\right)$. We apply the estimate (1.8ix) in each $U_{i}$ and sum, obtaining

$$
\begin{aligned}
{ }^{Z} E\left(\sum_{i}\left|h_{i}\right|^{p} \eta_{i} f\right)-c(\varepsilon, \delta) E(f) & \leq{ }^{h Z} E(f) \\
& \leq{ }^{z} E\left(\sum_{i}\left|h_{i}\right|^{p} \eta_{i} f\right)+c(\varepsilon, \delta) E(f) .
\end{aligned}
$$

This last estimate implies ${ }^{h Z} E(f)={ }^{Z} E\left(|h|^{p} f\right)$, which is equivalent to (1.8v). (The reason for the implication is that we may choose $c(\varepsilon, \delta), \varepsilon, \delta \rightarrow 0$ and covers $\left\{U_{i}\right\}$ with diameters approaching zero. Then the sums $\sum \|\left. h_{i}\right|^{p} \eta_{i} f$ converge uniformly to $|h|^{p} f$, and then continuity of the linear functional ${ }^{z} E$ gives the conclusion.) Finally, we study (1.8.1). Recall the definition of $e_{\varepsilon}(x)$ given in (1.2ii). Compare this to the approximant obtained using the exponential map:

$$
\tilde{e}_{\varepsilon}(x) \equiv \int_{S_{x}^{n-1}} \frac{d^{p}(u(x), u(\exp (x, \varepsilon \omega)))}{\varepsilon^{p}} d \sigma(\omega)
$$

Because of the uniform closeness of the respective surface measures, we see that

$$
(1-o(1)) e_{\varepsilon}(x) \leq \tilde{e}_{\varepsilon}(x) \leq(1+o(1)) e_{\varepsilon}(x)
$$

where $o(1)$ is a term which approaches zero uniformly (in $x$ ), as $\varepsilon \rightarrow 0$. Hence

$$
\tilde{e}_{\varepsilon}(x) d \mu \rightarrow d e
$$

We also note that for the fixed vector field $\omega$ (as defined above (1.8.1)), the two diffeomorphism families $\psi_{1}(x, t)=\bar{x}(x, t)$ and $\psi_{2}(x, t)=\exp (x, t \omega)$ have the same velocity vector field $\omega$ when $t=0$. In succession we apply (1.8xi), Fubini's Theorem, the Lebesgue Dominated Convergence Theorem (justified 
by $(1.8 \mathrm{vii}))$, and then (1.8ii) for the pair $\left(\psi_{1}, \psi_{2}\right)$ :

$$
\begin{aligned}
E(f) & =\lim _{\varepsilon \rightarrow 0} \int f(x) \tilde{e}_{\varepsilon}(x) d \mu(x) \\
& =\lim _{\varepsilon \rightarrow 0} \int\left(\int f(x) \frac{d^{p}(u(x), u(\exp (x, \varepsilon \omega)))}{\varepsilon^{p}} d \mu(x)\right) d \sigma(\omega) \\
& =\int \lim _{\varepsilon \rightarrow 0}\left(\int f(x) \frac{d^{p}(u(x), u(\exp (x, \varepsilon \omega)))}{\varepsilon^{p}} d \mu(x)\right) d \sigma(\omega) \\
& =\int \omega E(f) d \sigma(\omega) .
\end{aligned}
$$

This shows (1.8.1) and completes the proof of Theorem 1.8.1.

1.9. Differentiability theory for directional energies. For $p>1, u \in$ $W^{1, p}(\Omega, X)$, and $Z \in \Gamma(T \bar{\Omega})$ we show that the directional -derivative energies $d\left({ }^{Z} e\right)$ are absolutely continuous with respect to Lebesgue measure, i.e. $d\left({ }^{Z} e\right)=$ ${ }^{z} e(x) d \mu(x)$, for an $L^{1}$ energy-density function ${ }^{z} e(x)$. Furthermore, the $\varepsilon$ energy functions converge almost everywhere to ${ }^{Z} e(x)$. When $Z$ is non-zero one can always choose local coordinates so that $Z=\partial^{1}$. This motivates us to first consider the special configuration below:

(1.9i) Definition. Let $\Omega \subset \mathbb{R}^{n}$ be a bounded domain, and let $g$ be the Euclidean metric. Let $1 \leq p<\infty, u \in L^{p}(\Omega, X), \omega=\partial^{1}$, and let $u$ have finite $p$-energy in the direction $\omega$,

$$
E \equiv{ }^{\omega} E<\infty,
$$

and write $E(f)$ for the corresponding linear functional. (In the following discussion $\omega$ will be fixed and we will frequently suppress it in our notation.) Assume that each line in the direction of $\omega$ intersects $\Omega$ in at most one interval. Write $\Pi$ for the projection of $\Omega$ onto the $(n-1)$-plane $\left\{x^{1}=0\right\}$. Thus we may write

$$
\begin{aligned}
\Omega & =\left\{x=(t, y), y \in \Pi, t \in I_{y} \subset \mathbb{R}\right\} \\
E^{y} & =E\left(\left.u\right|_{I_{y}}\right) .
\end{aligned}
$$

That is, $E^{y}$ is the $p$-energy of the 1-variable map $\left.u\right|_{I_{y}}$ from $I_{y}$ to $X$. In case $E^{y}<\infty$ we write $E^{y}(f)$ for the corresponding linear functional on $C_{c}\left(I_{y}\right)$. For a function $f \in C_{c}(\Omega)$ we also write $f$ for its restriction fo $I^{y}$. 
Lemma 1.9.1. For the configuration (1.9i) we have

$$
E=\int E^{y} d y, \text { and } E(f)=\int E^{y}(f) d y, \text { for any } f \in C_{c}(\Omega)
$$

Conversely, if $u \in L^{p}(\Omega, X)$ is a map for which $\int E^{y} d y$ is finite, then $u$ has finite energy ${ }^{\omega} E$, and the above identities hold.

Proof. If the first part of the lemma is true, then the converse statement follows easily. Indeed, from Theorem 1.5.1 and (1.4vii) we have the following estimate for $f \geq 0, f \in C_{c}(\Omega)$ :

$$
E_{\varepsilon}(f)=\int E_{\varepsilon}^{y}(f) d y \leq \int E^{y}\left(f_{\varepsilon}^{0}\right) d y \leq\left(|f|_{\infty}+o(\varepsilon)\right) \int E^{y} d y
$$

Hence ${ }^{\omega} E<\infty$, and the first part of the lemma applies.

We now prove the first part of the Lemma. We use an absolutely continuous measure $\nu$ satisfying (1.2iv) to define our $\varepsilon$-energy densities. (For example, the choice $d \nu(\lambda)=(p+1) \lambda^{p}$ for $0<\lambda<1$ would correspond to interval averaging.) For any $f \in C_{c}(\Omega)$ with $0 \leq f \leq 1$, the fact that $E<\infty$ implies that given $\delta>0$ and $C \geq 0$, there exists $\varepsilon_{1}>0$ so that

$$
\lim _{\varepsilon^{\prime} \rightarrow 0} E_{\varepsilon^{\prime}}\left(f_{\varepsilon_{1}}^{C}\right) \leq E+\delta
$$

Since $E_{\varepsilon^{\prime}}\left(f_{\varepsilon}^{C}\right)=\int E_{\varepsilon^{\prime}}^{y}\left(f_{\varepsilon}^{C}\right) d y,(1.9 \mathrm{ii})$ and Fatou's Lemma imply

$$
\int \liminf _{\varepsilon^{\prime} \rightarrow 0} E_{\varepsilon^{\prime}}^{y}\left(f_{\varepsilon_{1}}^{C}\right) d y \leq E+\delta
$$

Fix $\delta, \varepsilon_{1}$ and set $C=0$. Denote the integrand above by $h\left(y, \varepsilon^{\prime}\right)$. By the absolute continuity of $\nu$, by Remark 1.4 .3 (and the fact that each $I_{y}$ is Euclidean),

$$
E_{\varepsilon}^{y}(f) \leq \liminf _{\varepsilon^{\prime} \rightarrow 0} h\left(y, \varepsilon^{\prime}\right), \text { for any } \varepsilon<\varepsilon_{1}
$$

Hence (taking the lim sup of the right-hand side and noting that $\delta$ was arbitrary)

$$
\int \limsup _{\varepsilon \rightarrow 0} E_{\varepsilon}^{y}(f) d y \leq E
$$


Pick any sequence $\left\{f_{i}\right\} \subset C_{c}(\Omega)$ which is monotone increasing in $i$ and converges (uniformly on compact subsets) to 1 , as $i \rightarrow \infty$. The monotone convergence theorem and (1.9iv) yield

$$
\int \sup _{0 \leq f \leq 1}\left(\limsup _{\varepsilon \rightarrow 0} E_{\varepsilon}^{y}(f) d y\right) \leq E
$$

i.e.

$$
\int E^{y} d y \leq E
$$

Thus for any $f \in C_{c}(\Omega)$

$$
E(f)=\lim _{\varepsilon \rightarrow 0} \int E_{\varepsilon}^{y}(f) d y=\int E^{y}(f) d y
$$

by the Lebesgue Dominated Convergence Theorem. Again using the sequence $\left\{f_{i}\right\}$ described above, this time in (1.9vi), shows that $E=\int E^{y} d y$.

Lemma 1.9.2. For the configuration (1.9i) there is a representative of $u$ having the property that for almost all $I_{y}=\left(a_{y}, b_{y}\right)$ with $y \in \Pi$,

$$
\begin{gathered}
\lim _{t \rightarrow a_{y}^{+}} u(t, y) \equiv u\left(a_{y}, y\right) \\
\lim _{t \rightarrow b_{y}^{-}} u(t, y) \equiv u\left(b_{y}, y\right)
\end{gathered}
$$

exist. In case $p>1$ (and for the $y$ above) $\left.u\right|_{I_{y}}$ is a Hölder continuous mapping, with exponent $\alpha=\frac{p-1}{p}$. In case $p=1$ the functions $\left.u\right|_{I_{y}}$ are maps of bounded variation. In all cases we have the estimate

$$
\int_{\Pi} d^{p}\left(u\left(a_{y}, y\right), u\left(b_{y}, y\right)\right) d y \leq{ }^{\omega} E \max _{y \in \Pi}\left(b_{y}-a_{y}\right)^{p-1} .
$$

Proof. We first deal with the case $p>1$ and then indicate how to modify the argument in case $p=1$. By Lemma 1.9.1 almost all $\left.u\right|_{I_{y}} \in W^{1, p}\left(I_{y}, X\right)$. For such $y$ write $\left.u\right|_{I_{y}}=v$, and assume (by rescaling) that $I_{y}=I=(0,1)$. For such a $v \in W^{1, p}(I, X)$ and $t \in I$, define $w(s)$ by

$$
w(s)=d(v(t+s), v(t)) \quad \text { for } s \in(-t, 1-t) .
$$

It follows from (1.6vii) of Corollary 1.6.3 that $w \in W^{1, p}((-t, 1-t), \mathbb{R})$, with $\left|\nabla_{s} w\right|^{p} d s \leq d e_{p}$ as measures. In particular (a representative of) $w \in C^{\alpha}((-t, 1-$ 
t)) (for $\alpha=\frac{p-1}{p}$ ), so is absolutely continuous. Therefore we have the estimate

$$
w(\varepsilon)-w(0) \leq \int_{0}^{\varepsilon}\left|\nabla_{s} w\right| d s \leq \int_{t}^{t+\varepsilon} e_{1}\left(t^{\prime}\right) d t^{\prime} .
$$

We would like to know that $w(0)=0$ and $w(\varepsilon)=d(u(t), u(t+\varepsilon))$ in the above estimate. This is formally true but actually requires some technical justification since a priori $v$ is only defined a.e. So, for any fixed $0<\mu<\frac{1}{2}$, we pick $f \in C_{c}(\Omega)$ with $0 \leq f \leq 1$ and with $f \equiv 1$ on the interval $(\mu, 1-\mu)$. From (1.4vii) and $p^{\prime}=1$ we have

$$
\int_{I} f(t)_{\nu} e_{\delta}(t) d t \leq \int_{I} e_{1}(t) d t<\infty
$$

for small $\delta>0$. Here we take $d \nu(\lambda)=2 \lambda$, corresponding to interval averages. Thus by Fatou's Lemma,

$$
\int_{\mu}^{1-\mu} \liminf _{\delta \rightarrow 0} e_{\delta}(t) d t<\infty
$$

so that

$$
\liminf _{\delta \rightarrow 0} \quad 2 \int_{0}^{\delta} \frac{d(v(t), v(t+s))}{\delta} \frac{d s}{\delta}<\infty \quad \text { a.e.t. }
$$

For such a $t$ we conclude that $w(0)=0$, since the numerator in the expression above is $w(s)$ and $w$ is Hölder continuous. For such a $t$ the estimate (1.9ix) implies

$$
d(v(t), v(t+\varepsilon)) \leq \int_{0}^{\varepsilon}\left|\nabla_{s} w\right| d s \leq \int_{t}^{t+\varepsilon} e_{1}\left(t^{\prime}\right) d t^{\prime} \quad \text { a.e. } \varepsilon \text {. }
$$

From the first half of this inequality we see that $v$ is equivalent to a $C^{\alpha}$ mapping, with $\alpha=\frac{p-1}{p}$ : By raising both sides to the $p^{\text {th }}$ power, using Hölder, and using the fact that $w \in W^{1, p}(\Omega, \mathbb{R})$ ), we have the estimate (at first a.e. $t, \varepsilon$, but then everywhere by redefinition)

$$
d^{p}(v(t), v(t+\varepsilon)) \leq\left(\int_{t}^{t+\varepsilon}\left|\nabla_{s} w\right|^{p}\right) \varepsilon^{p-1}
$$


This implies that for the $y \in \Pi$ with $\left.u\right|_{I_{y}} \in W^{1, p}(\Omega, X)$ the limits (1.9vii) exist, and furthermore that the estimate

$$
d^{p}\left(u\left(a_{y}, y\right), u\left(b_{y}, y\right)\right) \leq\left(b_{y}-a_{y}\right)^{p-1} E^{y}
$$

holds. Upon integration this yields (1.9viii).

In the case $p=1$ it is necessary to modify the argument slightly. Now the function $w(s)$ is equivalent to a function of bounded variation, so may be taken to be continuous from the left, with only a countable number of discontinuities, and so that all one-sided limits exist. We replace (1.9ix) with the estimate

$$
w\left(\varepsilon^{-}\right)-w\left(0^{+}\right) \leq \operatorname{Var}(0, \varepsilon) w \leq e_{1}((t, t+\varepsilon)),
$$

where the,+- superscripts refer to limits from the right and left respectively. For $t$ satisfying the finite liminf condition above, $w\left(0^{+}\right)=0$. For such $t$ we conclude

$$
\text { (1.9xiii) } d(v(t), v(t+\varepsilon))=w(\varepsilon) \leq \operatorname{Var}(0, \varepsilon) w \leq e_{1}((t, t+\varepsilon)) \quad \text { a.e. } \varepsilon .
$$

We redefine $v$ to be the limit from the left of $v\left(t^{\prime}\right)$, where $t^{\prime}$ satisfies (1.9xiii). It is straightforward to verify that this leaves $v$ unchanged at such $t^{\prime}$, and that it creates a function which is continuous from the left, and satisfies (1.9xiii) everywhere. In particular, this representative for $v$ will have only a countable number of discontinuities, exactly at points where the measure $e_{1}$ has point masses. We deduce that the map $v$ is a map of bounded variation in the classical sense. In particular, all one sided limits exist. Also, returning to the intervals $I_{y}$ with $E^{y}<\infty$ we get the estimate

$$
d\left(u\left(a_{y}, y\right), u\left(b_{y}, y\right)\right) \leq E^{y} .
$$

Upon integration this yields (1.9viii), and the proof of Lemma 1.9.2 is complete.

Lemma 1.9.3. Let $1<p<\infty, u \in W^{1, p}(I, X)$. Then (its Hölder continuous representative satisfies)

$$
\lim _{\varepsilon \rightarrow 0} \frac{d(u(t), u(t+\varepsilon))}{\varepsilon}=e_{1}(t) \quad \text { a.e. }
$$


Proof. We see immediately from the inequality between the first and last terms of $(1.9 \mathrm{x})$ (and the Lebesgue Differentiation Theorem applied to the $L^{1}$-function $\left.e_{1}(t)\right)$ that

$$
\limsup _{\varepsilon \rightarrow 0} \frac{d(u(t), u(t+\varepsilon))}{\varepsilon} \leq e_{1}(t) \quad \text { a.e.t. }
$$

Now let $\delta>0$. Define

$$
\begin{aligned}
S_{\delta} \equiv & \left\{t \in I \text { s.t. } t \text { is a Lebesgue point for } e_{1},\right. \text { and } \\
& \left.\liminf _{\varepsilon \rightarrow 0} \frac{d(u(t), u(t+\varepsilon))}{\varepsilon}<e_{1}(t)-\delta\right\} .
\end{aligned}
$$

By definition, for any fixed $\mu>0$ we may cover $S_{\delta}$ by intervals $(t-\varepsilon, t+\varepsilon)$ with $\varepsilon<\mu$ and for which $t \in S_{\delta}$,

$$
\begin{aligned}
& \frac{d(u(t), u(t+\varepsilon))}{\varepsilon}<e_{1}(t)-\delta, \\
& e_{1}(t)-\frac{1}{\varepsilon} \int_{t}^{t+\varepsilon} e_{1}(s) d s<\frac{\delta}{2} .
\end{aligned}
$$

By a well-known covering lemma [Rudin, Lemma 8.4] we may pick a finite disjoint subset of this cover, with the finite sum of the interval lengths at least $\frac{1}{4}^{\text {th }}$ the measure of $S_{\delta}$. Denote the corresponding half-intervals by

$$
\left\{I_{i}\right\}_{i=1}^{k}, \quad I_{i}=\left[t_{i}, t_{i}+\varepsilon_{i}\right) .
$$

Complete this finite collection of intervals to a partition of $[0,1]$, by adding intervals

$$
\left\{J_{i^{\prime}}\right\}_{i^{\prime}=1}^{k^{\prime}}, \quad J_{i^{\prime}}=\left[t_{i^{\prime}}, t_{i^{\prime}}+\varepsilon_{i^{\prime}}\right),
$$

also with maximum length bounded by $\mu$. We have from $(1.9 \mathrm{x})$ and our covering hypothesis that

$$
\begin{aligned}
& \sum_{i} d\left(u\left(t_{i}\right), u\left(t_{i}+\varepsilon_{i}\right)\right)+\sum_{i^{\prime}} d\left(u\left(t_{i^{\prime}}\right), u\left(t_{i^{\prime}}+\varepsilon_{i^{\prime}}\right)\right) \\
& \quad \leq \sum_{i}\left(\int_{I_{i}} e_{1}(t) d t-\frac{\delta}{2} \varepsilon_{i}\right)+\sum_{i^{\prime}} \int_{J_{i^{\prime}}} e_{1}(t) d t \\
& \quad \leq \int_{I} e(t) d t-\frac{\delta}{8}\left|S_{\delta}\right| .
\end{aligned}
$$


If we can show

$$
\int_{I} e_{1}(t) d t=\lim _{\|P\| \rightarrow 0} \sum d\left(u\left(t_{i+1}\right), u\left(t_{i}\right)\right)
$$

for arbitrary partitions $P$ of $I$, we conclude that the measure of $S_{\delta}$ is 0 by letting $\mu \rightarrow 0$ in $(1.9 \mathrm{x})$. Picking a sequence $S_{\delta_{i}}$ with $\delta_{i} \rightarrow 0$ then implies Lemma 1.9.3. We verify (1.9xvi) as follows:

Because $d e_{1}=e_{1}(t) d t$ is absolutely continuous it is easy to see that the total $p=1$ energy $E_{1}$ for the map $u$ is given by

$$
\begin{aligned}
\int_{I} e_{1}(t) d t & =\lim _{\varepsilon \rightarrow 0} \int_{0}^{1-\varepsilon} \frac{d(u(t), u(t+\varepsilon))}{\varepsilon} d t \\
& =\lim _{\varepsilon \rightarrow 0} \int_{0}^{\varepsilon} \sum_{i=0}^{\left[\frac{1}{\varepsilon}\right]-2} \frac{d(u(t+i \varepsilon), u(t+(i+1) \varepsilon))}{\varepsilon} d t \\
& \equiv \lim _{\varepsilon \rightarrow 0} \frac{1}{\varepsilon} \int_{0}^{\varepsilon} \sum_{\varepsilon}(t) d t .
\end{aligned}
$$

Hence for $\delta>0, \varepsilon>0$ small, there exists $t_{\varepsilon} \in(0, \varepsilon)$ with $\sum_{\varepsilon}\left(t_{\varepsilon}\right) \geq E_{1}-\delta$. Now let

$$
P: 0=t_{0}<t_{1}<\ldots<t_{k}=1
$$

be an arbitrary partition of $I$, subject to $N\|P\|<\varepsilon$ for $N$ large. We pick a subpartition $P^{\prime} \subset P$ for which $t_{i}$ is within $\frac{\varepsilon}{N}$ of $t_{\varepsilon}+i \varepsilon, i=1, \ldots\left(\left[\frac{1}{\varepsilon}\right]-2\right)$. Note that this implies

$$
\sum_{i}\left|t_{\varepsilon}+i \varepsilon-t_{i}\right| \leq \frac{1}{N}
$$

Hence

$$
\begin{aligned}
\sum_{P} d\left(u\left(t_{j}\right), u\left(t_{j+1}\right)\right) & \geq \sum_{P^{\prime}} d\left(u\left(t_{i}\right), u\left(t_{i+1}\right)\right) \\
& \geq \sum_{\varepsilon}\left(t_{\varepsilon}\right)-2 \sum\left|\int_{t_{i}}^{t_{\varepsilon}+i \varepsilon} e_{1}(t) d t\right| \\
& \geq \int_{I} e_{1}(t) d t-\delta-2\left(\frac{1}{N}\right)^{\frac{p-1}{p}}\left(E_{p}\right)^{\frac{1}{p}}
\end{aligned}
$$

(where we have used Remark in the last step). As $\delta$ and $N$ are arbitrary, and since a one-sided estimate suffices (see $(1.9 \mathrm{x})$ ), we conclude $(1.9 \mathrm{xvi})$ and the proof of Lemma 1.9.3 is complete. 
Lemma 1.9.4. Let $(\Omega, g), \omega$ and $u$ be as in (1.9i). Let $1<p<\infty$. Then for any $1 \leq p^{\prime}<p$ the energy-density function $e_{p^{\prime}}(x)$ (for the direction $\omega$ ) satisfies

$$
e_{p^{\prime}}(x)=\left(e_{1}(x)\right)^{p^{\prime}} \quad \text { a.e. }
$$

Furthermore, there exists a representative of $u$ so that

$$
\lim _{\varepsilon \rightarrow 0} \frac{d^{p^{\prime}}(u(t, y), u(t+\varepsilon, y))}{\varepsilon^{p^{\prime}}}=e_{p^{\prime}}(t, y) \quad \text { a.e. }
$$

Proof. From Lemma 1.9.1 and Lemma 1.9.3 we know we may pick a representative of $u$ so that

$$
\lim _{\varepsilon \rightarrow 0} \frac{d^{p^{\prime}}(u(t, y), u(t+\varepsilon, y))}{\varepsilon^{p^{\prime}}}=\left(e_{1}(t, y)\right)^{p^{\prime}} \quad \text { a.e. }
$$

so $(1.9 x v i i i)$ will follow from $(1.9 x v i i)$. The claim $(1.9 x v i i)$ is true because of the following measure theory lemma.

Lemma. Let $1<p<\infty,\left\{g_{\varepsilon}\right\} \subset L_{\text {loc }}^{p}(\Omega, \mathbb{R}), g_{\varepsilon} \geq 0$. Suppose that on any compact subset of $\Omega$ the $L^{p}$-norms of $g_{\varepsilon}$ are uniformly bounded as $\varepsilon \rightarrow 0$. Let $g_{\varepsilon}(x) \rightarrow g(x)$ a.e. as $\varepsilon \rightarrow 0$. If, for some $1 \leq p^{\prime}<p$ it is also true that $g_{\varepsilon}^{p^{\prime}} d \mu \rightarrow h d \mu$ with $h \in L^{1}(\Omega, \mathbb{R})$, then in fact $h=g^{p^{\prime}}$ a.e.

Proof. Let $f \in C_{c}(\Omega)$. Write $\Omega=G \cup B, G \cap B=\varnothing$, where $g_{\varepsilon} \rightarrow g$ uniformly on $G$. (So $B$ can be chosen with arbitrarily small positive measure). Then

$$
\lim _{\varepsilon \rightarrow 0} \int_{G} g_{\varepsilon}^{p^{\prime}} f d \mu=\int_{G} f g^{p^{\prime}} d \mu
$$

and

$$
\limsup _{\varepsilon \rightarrow 0} \int_{B} g_{\varepsilon}^{p^{\prime}} f d \mu \leq|f|_{\infty} C^{\frac{p^{\prime}}{p}}(\mu(B))^{\frac{p-p^{\prime}}{p}},
$$

where $C$ depends on the uniform $L^{p}$ estimates for the $g_{\varepsilon}$ as $\varepsilon \rightarrow 0$, on the compact subset $\operatorname{supp}(f)$. For fixed $f$ we may make this last term smaller than any given $\delta>0$ by picking $\mu(B)$ small. Combining this observation with Fatou's Lemma gives

$$
\int_{\Omega} f g^{p^{\prime}} d \mu \leq \int_{\Omega} f h d \mu=\lim _{\varepsilon \rightarrow 0} \int_{\Omega} f g_{\varepsilon}^{p^{\prime}} d \mu \leq \int_{\Omega} f g^{p^{\prime}} d \mu+\delta .
$$

This proves the Lemma. 
By applying this result to the functions

$$
\begin{aligned}
g_{\varepsilon}(t, y) & =\frac{d(u(t, y), u(t+\varepsilon, y))}{\varepsilon} \\
h(x) & =e_{p^{\prime}}(x),
\end{aligned}
$$

we immediately conclude (1.9xvii) and Lemma 1.9.4.

Lemma 1.9.5. Let $(\Omega, g), \omega$ and $u$ be as in (1.9i). Let $1<p<\infty$. Then the energy density measure de (for the direction $\omega$ ) is absolutely continuous with respect to Lebesgue measure, de $=e_{p}(x) d \mu(x)$, and

$$
e_{p}(x)=e_{1}(x)^{p} \quad \text { a.e. }
$$

Furthermore, there is a representative of $u$ so that

$$
\lim _{\varepsilon \rightarrow 0} \frac{d^{p}(u(t, y)), u(t+\varepsilon, y)}{\varepsilon^{p}}=e_{p}(t, y) \quad \text { a.e. }
$$

For any representative of $u$, and any choice of $\nu$ in (1.2iv) for which $\lambda^{-p} d \nu(\lambda)$ is a bounded multiple of $d \lambda$, we have

$$
\lim _{\varepsilon \rightarrow 0} e_{\varepsilon}(x)=e_{p}(x) \quad \text { a.e. }
$$

Proof. As in Lemma 1.9.4 it suffices to show the first claim only. Note that the third claim will follow from the second, because if we change representatives for $u$, almost all $\left.u\right|_{I_{y}}$ will remain the same $L^{p}$ function, and for such $y$ the ${ }_{\nu} e_{\varepsilon}(t, y)$ will be unchanged, because of the restriction on $\nu$. We establish the first claim by verifying the two measure inequalities

$$
\begin{aligned}
& e_{1}^{p} d \mu \leq d e, \\
& d e \leq e_{1}^{p} d \mu .
\end{aligned}
$$

The first of these follows immediately from Fatou's Lemma:

Fix $f \in C_{c}(\Omega)$, then

$$
\begin{aligned}
\int f e_{1}^{p} d \mu & =\int \liminf _{\varepsilon \rightarrow 0} f \frac{d^{p}(u(t, y), u(t+\varepsilon, y))}{\varepsilon^{p}} d \mu \\
& \leq \int_{\Omega} f d e .
\end{aligned}
$$


To show the second we again fix $f \in C_{c}(\Omega)$. Then

$$
\int_{\Omega} f d e=\lim _{\varepsilon \rightarrow 0} \int_{\Omega} f \frac{d^{p}}{\varepsilon^{p}} d \mu,
$$

where we suppress the various arguments $x=(t, y), u(t, y), u(t+\varepsilon, y)$.

Hence

$$
E(f)=\int_{\Omega} f \frac{d^{p}}{\varepsilon^{p}} d \mu+\delta_{1}(\varepsilon)
$$

where $\delta_{1}(\varepsilon) \rightarrow 0$ as $\varepsilon \rightarrow 0$. But for $\varepsilon>0$ fixed,

$$
\int_{\Omega} f \frac{d^{p}}{\varepsilon^{p}} d \mu=\int_{\Omega} f \frac{d^{p^{\prime}}}{\varepsilon^{p^{\prime}}} d \mu+\delta_{2}\left(p^{\prime}\right)
$$

where $\delta_{2}\left(p^{\prime}\right) \rightarrow 0$ as $p^{\prime} \rightarrow p$. Applying the estimate (1.4vii) to the integral on the right side, and also Lemma 1.9.4, yields

$$
\begin{aligned}
E(f) & =\int_{\Omega} f \frac{d^{p^{\prime}}}{\varepsilon^{p^{\prime}}} d \mu+\delta_{1}(\varepsilon)+\delta_{2}\left(p^{\prime}\right) \\
& \leq \int_{\Omega} f_{\varepsilon}^{C}(x) e_{1}(x)^{p^{\prime}} d \mu(x)+\delta_{1}(\varepsilon)+\delta_{2}\left(p^{\prime}\right) .
\end{aligned}
$$

Using the already established inequality $\left(e_{1}\right)^{p} d \mu \leq d e$ to justify Lebesgue's Dominated Convergence Theorem, we let $p^{\prime} \rightarrow p$ and deduce

$$
\int_{\Omega} f d e \leq \int_{\Omega} f_{\varepsilon}^{C} e_{1}(x)^{p} d \mu+\delta_{1}(\varepsilon) .
$$

Letting $\varepsilon \rightarrow 0$ we conclude the desired second inequality $d e \leq\left(e_{1}^{p}\right) d \mu$. Thus Lemma 1.9 .5 is shown.

Theorem 1.9.6. Let $(\Omega, g)$ be a Riemannian domain (1.1). Let

$$
u \in W^{1, p}(\Omega, X) \text { for some } 1<p<\infty,
$$

and let $Z \in \Gamma(T \bar{\Omega})$. Then the energy-density measure $d^{Z}$ e for each $1 \leq p^{\prime} \leq p$ is absolutely continuous with respect to Lebesgue measure. In particular, if we denote the $p=1$ energy-density function by $\left|u_{*}(Z)\right|(x)$, then the $p^{\prime}$-energy measure is given by

$$
\left|u_{*}(Z)\right|^{p^{\prime}} d \mu_{g}(x)
$$


for each $1 \leq p^{\prime} \leq p$. For any choice of $\nu$ in (1.2iv) for which $\lambda^{-p} d \nu(\lambda)$ is a bounded multiple of $d \lambda$ we have (for each $1 \leq p^{\prime} \leq p$ )

$$
\lim _{\varepsilon \rightarrow 0} \underset{\nu}{Z} e_{\varepsilon}(x)=\left|u_{*}(Z)\right|^{p^{\prime}}(x) \quad \text { a.e. }
$$

Proof. We pick a local coordinate chart and express $Z=Z^{i} \partial_{i}$ in terms of the local coordinate direction fields. It suffices to prove our claim in the case $p^{\prime}=p$. From the triangle inequality (1.8iv) and the homogeneity property $(1.8 \mathrm{v})$ we may estimate

$$
{ }^{z} E(f) \leq|Z|_{\infty}^{p} n^{p} \sum_{i=1}^{n} \partial_{i} E(f)
$$

Applying Lemma 1.9.5 this yields

$$
d\left({ }^{Z} e\right) \leq|Z|_{\infty}^{p} n^{p} \sum_{i=1}^{n}\left|u_{*}\left(\partial_{i}\right)\right|^{p}(x) d \mu(x) .
$$

Since the Euclidean measure $d \mu(x)$ and the metric measure $d \mu_{g}(x)$ are uniformly equivalent, this last inequality proves the absolute continuity of $d^{Z} e$.

To prove the pointwise convergence of the ${ }_{\nu}^{Z} e_{\varepsilon}(x)$ to $\left|u_{*}(Z)\right|^{p}(x)$ we note first that (1.9xix) implies $\left|u_{*}(Z)\right|^{p}(x)=0$ a.e on $\{x \mid Z(x)=0\}$. All approximate energies ${ }_{\nu}^{Z} e_{\varepsilon}(x)$ are also zero on this set. Thus we need only verify the convergence statement on $\{x \mid Z(x) \neq 0\}$. Here we may do a $C^{1,1}$ change of coordinates from an initial local coordinate chart, turning $Z$ into a coordinate direction. The result then follows from Lemma 1.9.5, and the uniform equivalence of the respective volume measures.

\subsection{Absolute continuity of $d e$ for $p>1$.}

Theorem 1.10. Let $(\Omega, g)$ be a Riemannian domain (1.1i), and let $1<p<$ $\infty$. Let $u \in W^{1, p}(\Omega, X)$. Then the energy density measure de is absolutely continuous with respect to Lebesgue measure, i.e. there exists $|\nabla u|_{p}(x) \in$ $L^{1}(\Omega, \mathbb{R}) \quad$ s.t.

$$
d e=|\nabla u|_{p}(x) d \mu(x) .
$$

Proof. (We will explain the why we use a subscript rather than a superscript for $p$ after the proof.) We reduce to the case where $\Omega$ has a local orthonormal 
frame, as in (1.8.1). Thus we have that equality, which we reproduce here:

$$
E(f)=\int_{S^{n-1}}{ }^{\omega} E(f) d \sigma(\omega) .
$$

By Theorem 1.9.6, we may express the corresponding energy-density measures by

$$
d\left({ }^{\omega} e\right)=\left|u_{*}(\omega)\right|^{p}(x) d \mu(x)
$$

so (1.10i) may be rewritten as

$$
E(f)=\int_{S^{n-1}} \int_{\Omega}\left|u_{*}(\omega)\right|^{p}(x) f(x) d \mu(x) d \sigma(\omega) .
$$

It is easy to check that the non-negative functions $\left|u_{*}(\omega)\right|^{p}(x)$ are jointly measurable in $(x, \omega)$. Applying the monotone convergence theorem to an increasing sequence $\left\{f_{i}\right\} \subset C_{c}(\Omega)$ for which $f_{i} \rightarrow 1$ uniformly on compact subsets of $\Omega$, we deduce

$$
\int_{S^{n-1}} \int_{\Omega}\left|u_{*}(\omega)\right|^{p}(x) d \mu(x) d \sigma(\omega)=E<\infty .
$$

Fubini's theorem then implies

$$
E(f)=\int_{\Omega} f(x)\left(\int_{S^{n-1}}\left|u_{*}(\omega)\right|^{p}(x) d \sigma(\omega)\right) d \mu(x)
$$

for the $L^{1}$ function

$$
|\nabla u|_{p}(x) \equiv \int_{S^{n-1}}\left|u_{*}(\omega)\right|^{p}(x) d \sigma(\omega)
$$

The reason why $p$ is placed as a subscript in the notation $|\nabla u|_{p}$ is to prevent confusion about the relation of different $p$-energies: It is not true (unless $X=$ $\mathbb{R}$ ) that $|\nabla u|_{p}$ is equal (even up to a constant multiple) to $|\nabla u|_{p^{\prime}}^{p / p^{\prime}}$. (Of course if both expressions make sense, then they will be uniformly equivalent.) Because we will have a special interest in the case $p=2$ in the second chapter, we will define

$$
|\nabla u|^{2}(x)=\frac{1}{\omega_{n}}|\nabla u|_{2}(x)=\frac{1}{\omega_{n}} \int_{S^{n-1}}\left|u_{*}(\omega)\right|^{2}(x) d \sigma(\omega)
$$


for $u \in W^{1,2}(\Omega, X)$. We will see in Section 2 that this definition is consistent with the usual way of defining $|d u|^{2}$ for maps between Riemannian manifolds. (See also (1.2viii).)

1.11. The calculus of energy-density functions. We collect some useful Calculus facts about our various energy-density functions.

Theorem 1.11. Let $u \in W^{1, p}(\Omega, X)$ for some $1<p<\infty$. If $Z, W \in \Gamma(T \bar{\Omega})$ and if $h \in C^{0,1}(\bar{\Omega})$, then

$$
\begin{aligned}
\left|u_{*}(Z+W)\right| & \leq\left|u_{*}(Z)\right|+\left|u_{*}(W)\right| \\
\left|u_{*}(h Z)\right|^{p} & =|h|^{p}\left|u_{*}(Z)\right|^{p} \\
\left|u_{*}(Z)\right|^{p} & \leq C(n)|Z|^{p}|\nabla u|_{p} .
\end{aligned}
$$

If $\psi$ is a $C^{1,1}$ diffeomorphism from $\bar{\Omega}_{1}$ to $\bar{\Omega}$, and if we write $v=u \circ \psi$, then $v \in W^{1, p}\left(\Omega_{1}, X\right)$, and the chain rule

$$
\left|v_{*}(Z)\right|^{p}=\left|u_{*}\left(\psi_{*}(Z)\right)\right|^{p}
$$

holds. If two metrics are close, then their Sobolev energy-density functions are close. In particular, let $\Omega \subset \mathbb{R}^{n}$ and let $\delta, g$ be the Euclidean and a Riemannian metric on $\Omega$. Denote the two corresponding energy-density functions of $u$ by $|\nabla u|_{p}$ and $|\nabla u|_{p, g}$, and let $d \mu$ and $d \mu_{g}$ be the two induced volume forms. Let $\lambda^{2}$ and $\Lambda^{2}$ be the minimum and maximum eigenvalues of $g$ (relative to $\delta$ ), respectively. Then we have the estimate

$$
\frac{\lambda^{2 n}}{\Lambda^{n+p}}|\nabla u|_{p} d \mu \leq|\nabla u|_{p, g} d \mu_{g} \leq \frac{\Lambda^{2 n}}{\lambda^{n+p}}|\nabla u|_{p} d \mu .
$$

Proof. The statements (1.11i) are restatements of corresponding claims from Theorem 1.8.1, using the subsequent differentiability results of Theorem 1.9.6 and Theorem 1.10. Note that we have replaced the supremum norm $|Z|_{\infty}$ with the pointwise norm $|Z|_{\infty}$ in the third inequality. This is easily justified by using (1.8.i) and a partition of unity argument.

For $\psi$ as above it is clear (say using ball averages) that $v=u \circ \psi \in$ $W^{1, p}\left(\Omega_{1}, X\right)$. The chain rule (1.11ii) follows from the fact that the $\varepsilon$ energydensity functions satisfy

$$
{ }_{\nu}^{Z} e_{\varepsilon}(x)={ }_{\nu}^{\psi_{*}} Z e_{\varepsilon}(\psi(x))
$$


and that for appropriate $\nu$ these converge almost everywhere as $\varepsilon \rightarrow 0$ to the corresponding directional-energy functions (Theorem 1.9.6).

We prove the claim (1.11iii) as follows. Recall from 1.2 that computing an $\varepsilon$-energy with radius $\varepsilon R$ ball averaging corresponds to the choice

$$
d \nu(\rho)=\frac{n+p}{R^{n+p}} \rho^{n+p-1} d \rho \quad 0<\rho<R .
$$

Call the measure above $\nu_{R}$. Then the $\varepsilon$-approximate energy density function (with respect to a metric $g$ ) is given by

$$
{ }_{\nu_{R}} e_{\varepsilon, g}(x)=\frac{n+p}{R^{n+p}} \frac{1}{\varepsilon^{n}} \int_{B(x, \varepsilon R)} \frac{d^{p}(u(x), u(y))}{\varepsilon^{p}} d \mu_{g}(y)
$$

In our case we have the inequalities

$$
\lambda^{n} d \mu \leq d \mu_{g} \leq \Lambda^{n} d \mu
$$

and the ball containments

$$
B\left(x, \frac{\varepsilon}{\Lambda}\right) \subset B(x, \varepsilon)_{g} \subset B\left(x, \frac{\varepsilon}{\lambda}\right) .
$$

Letting $r=\frac{1}{\Lambda}$ and $R=\frac{1}{\lambda}$ we deduce that

$$
\frac{\lambda^{n}}{\Lambda^{n+p}} \nu_{r} e_{\varepsilon}(x) \leq{ }_{\nu_{1}} e_{\varepsilon, g}(x) \leq \frac{\Lambda^{n}}{\lambda^{n+p}} \nu_{R} e_{\varepsilon}(x) .
$$

Upon integration (and applying the volume inequalities again) we see that (for any $\left.f \geq 0, f \in C_{c}(\Omega)\right)$

$$
\frac{\lambda^{2 n}}{\Lambda^{n+p}} \nu_{r} E_{\varepsilon}(f) \leq{ }_{\nu_{1}} E_{\varepsilon, g}(f) \leq \frac{\Lambda^{2 n}}{\lambda^{n+p}} \nu_{R} E_{\varepsilon}(f) .
$$

¿From the weak convergence properties of our $\varepsilon$-energy functionals, we deduce that the limit measures satisfy the same inequalities, i.e.

$$
\frac{\lambda^{2 n}}{\Lambda^{n+p}}|\nabla u|_{p} d \mu \leq|\nabla u|_{p, g} d \mu_{g} \leq \frac{\Lambda^{2 n}}{\lambda^{n+p}}|\nabla u|_{p} d \mu .
$$

This is exactly the claim (1.11iii). 
1.12. Trace theory for Lipschitz domains. In this section we develop the $L^{p}$ trace theory for finite-energy maps defined on Lipschitz domains. We show that two functions have the same trace if and only if the distance between them is a real-valued function of finite energy, having zero trace on the boundary. We also prove a replacement theorem: If $\Omega$ can be partitioned into two Lipschitz subdomains, and if there are finite-energy maps for each subdomain whose traces agree on the common boundary, then together they define a global map of finite energy on all of $\Omega$, and the total energy of this map is the sum of the energies of its components.

DEFinition. If $(\Omega, g)$ is a Riemannian domain then the usual way of saying that $\partial \Omega$ is Lipschitz near $x \in \partial \Omega$ is to require that there be a neighborhood $U$ of $x$ and a local coordinate chart on $U$ so that in these coordinates $\partial \Omega \cap U$ is the graph of a Lipschitz function above some $(n-1)$-dimensional hyperplane. It is easy to see that this definition is equivalent to the following one, which requires the existence of suitable transverse vector fields. We will say that $\partial \Omega$ is Lipschitz near $x \in \partial \Omega$ if there is a neighborhood $U$ of $x$, a smooth vector field $Z$ defined on $U$, and positive numbers $\rho, t_{0}$ so that for all $x \in \partial \Omega$ the flow $\bar{x}(x, t)$ in 1.1 satisfies

$$
\begin{aligned}
& \bar{x}(x, t) \in \Omega \quad \text { if } 0<t<t_{0}, \\
& \bar{x}(x, t) \notin \Omega \quad \text { if }-t_{0}<t<0, \\
& d(\bar{x}(x, t), \partial \Omega)>\rho|t| \quad \text { if }|t|<t_{0} .
\end{aligned}
$$

We will say that a compact subset $\Gamma$ of $\partial \Omega$ is Lipschitz if $\partial \Omega$ is Lipschitz near each $x \in \Gamma$. It is easy to see that this is equivalent to the existence of a smooth vector field $Z$ defined in a neighborhood of $\Gamma$ which satisfies (1.12i) for some choice of positive numbers $\rho, t_{0}$. We will say that $\Omega$ itself is a Lipschitz domain if $\partial \Omega$ is Lipschitz.

Let $1 \leq p<\infty, u \in W^{1, p}(\Omega, X)$ if $p>1(u \in B V(\Omega, X)$ if $p=1)$. If $\Gamma \subset \partial \Omega$ is Lipschitz, and if $Z$ is a transverse vector field satisfying (1.12i) for all $x \in \Gamma$, then there is a natural way to define a trace map $u \in L^{p}(\Gamma, X)$. as follows. Recall that we write $\bar{x}(x, t)$ for the flow induced by $Z$ (1.1). By using Lemma 1.9.2 on a finite number of local coordinate charts (for which $Z$ corresponds to the direction $\omega$ in the configuration (1.9i), we see that $u$ has 
a representative so that for almost all $x \in \Gamma$ the maps $u\left(\bar{x}(x, t), 0<t, t_{0}\right.$ are either Hölder continuous $(p>1)$ or of bounded variation $(p=1)$. Thus the map

$$
u(x) \equiv \lim _{t \rightarrow 0^{+}} u(\bar{x}(x, t))
$$

is defined almost everywhere (with respect to $(n-1)$-dimensional Hausdorff measure on $\Gamma$ ). Furthermore, we deduce from the estimate (1.9viii) and from Theorem 1.8.1 that (for a constant $C$ depending on $\Omega$ )

$$
\int_{\Gamma} d^{p}(u(x), u(\bar{x}(x, t))) d \Sigma_{x}^{n-1} \leq C\left(\int_{\Omega_{t|Z| \infty}^{C}} d e\right)\left(t|Z|_{\infty}\right)^{p-1}
$$

(Here we use the notation $\Omega_{\varepsilon}^{C}$ for the complement in $\Omega$ of $\Omega_{\varepsilon}$, i.e. those points in $\Omega$ whose distance to $\partial \Omega$ is at most $\varepsilon$.) Because $u \in L^{p}(\Omega, X)$, for almost all t the maps $u(\bar{x}(x, t))$ are in $L^{p}(\partial \Omega, X)$. We conclude from (1.12ii) that the trace map is the $L^{p}$ limit of the maps $u(\bar{x}(x, t))$ as $t \rightarrow 0$, so is itself an $L^{p}$ map. Furthermore, if we use any representative for $u$ it follows that the trace map is the $L^{p}$ limit of almost all of the maps $u(\bar{x}(x, t)$, as $t \rightarrow 0$, and so is well-defined independently of our representative for $u$.

Lemma 1.12.1. The definition of trace given above is independent of choice of transverse vector field $Z$. Also, if $\Gamma_{1} \subset \Gamma$, then the trace of $u$ on $\Gamma_{1}$ is the restriction to $\Gamma_{1}$ of the trace of $u$ on $\Gamma$.

Proof. The second claim follows from the first one. The first claim is a consequence of Lemma 1.8.2 and the following argument. Let $Z$ and $W$ be two transverse vector fields satisfying $(1.12 \mathrm{i})$. Let $\bar{x}_{1}(x, t)$ and $\bar{x}_{2}(x, t)$ be the corresponding flows. We wish to show that the maps $u\left(\bar{x}_{1}(x, t)\right)$ and $u\left(\bar{x}_{2}(x, t)\right.$ (from $\partial \Omega$ to $X$ ) converge (a.e.t) to the same trace function. Denote the map which sents $\bar{x}_{1}(x, t)$ to $\bar{x}_{2}(x, t)$ by $\psi$. Note that $\psi$ is a bi-Lipschitz mapping of 
a neighborhood of $\Gamma$ to its image. Thus we may estimate

$$
\begin{aligned}
& \int_{\frac{\mu_{1}}{\rho}}^{\frac{\mu_{2}}{|Z|_{\infty}}} \int_{\partial \Omega} d^{p}\left(u\left(\bar{x}_{1}(x, t)\right), u\left(\bar{x}_{2}(x, t)\right)\right) d \Sigma_{x} d t \\
& \leq C \int_{\Omega_{\mu_{2}}} f(x) d^{p}(u(x), u(\psi(x))) d \mu(x),
\end{aligned}
$$

where $f \in C_{c}\left(\Omega_{\mu_{2}+\delta}^{C}\right)$ satisfies $0 \leq f \leq 1$, and $f \equiv 1$ for $x$ satisfying

$$
\mu_{1} \leq d(x, \partial \Omega) \leq \mu_{2} .
$$

Write $\delta$ for the supremum of $|\psi(x)-x|$, over the subset $U \cap \Omega_{\mu_{2}}^{C}$. Then for small $\mu_{2}$ we see from continuous dependence on parameters that

$$
\delta \leq C \mu_{2} \max _{\Gamma}|Z-W| .
$$

Pick $\mu_{1}$ to satisfy $\frac{\mu_{1}}{\rho}=\frac{\mu_{2}}{2|Z|_{\infty}}$. In case $|Z-W|$ is sufficiently small we may guarantee that $3 \delta<\mu_{1}$ (for all small $\mu_{2}$ ). In that case we may construct a suitable $f$ for (1.11iii) which also satisfies the condition necessary to apply Lemma 1.8.2, namely $f \in C_{c}\left(\Omega_{3 \delta}\right)$. From the Lemma we see

$$
\int_{\Omega_{\mu_{2}}^{C}} f(x) d^{p}(u(x), u(\psi(x))) d \mu(x) \leq C \delta^{p} \int_{\Omega_{\mu_{2}+\delta}^{C}} d e .
$$

From the combination of (1.12iii) and (1.12iv) we see that the average values of the integrals of $d^{p}\left(u\left(\bar{x}_{1}(x, t)\right), u\left(\bar{x}_{2}(x, t)\right)\right)$ (with respect to $\frac{\mu_{2}}{2|Z|_{\infty}}<t<\frac{\mu_{2}}{|Z|_{\infty}}$ ) converge to 0 as $\mu_{2} \rightarrow 0$. Thus $Z$ and $W$ define the same trace function on $\Gamma$, if the closeness assumption above is satisfied. Since the set of strictly transverse vector fields is a positive cone (i.e. if $Z$ and $W$ are suitable, then so are $a Z+b W$ for positive constants $a, b$ ), it is connected. Hence it suffices to prove the lemma for sufficiently close vector fields, as we have done.

Theorem 1.12.2. Let $(\Omega, g)$ be a Lipschitz Riemannian domain and let $1<$ $p<\infty$. Any $u \in W^{1, p}(\Omega, X)$ has a well-defined trace map $u$ (or $\operatorname{tr}(u)$ ), with $\operatorname{tr}(u) \in L^{p}(\partial \Omega, X)$. If the sequence $\left\{u_{i}\right\} \subset W^{1, p}(\Omega, X)$ has uniformly bounded energies $E^{u_{i}}$, and if $\left\{u_{i}\right\}$ converges in the $L^{p}$ distance to a map $u$, then the trace functions of the $u_{i}$ converge in $L^{p}(\partial \Omega, X)$ to the trace of $u$. Two functions 
$u, v \in W^{1, p}(\Omega, X)$ have the same trace if and only if $d(u, v) \in W^{1, p}(\Omega, \mathbb{R})$ has trace zero.

Proof. That $u \in W^{1, p}(\Omega, X)$ has a well-defined trace $\operatorname{tr}(u) \in L^{p}(\partial \Omega, X)$ follows from Lemma 1.12.1 and the remarks preceding it. Furthermore, if we fix a transverse vector field $Z$ satisfying (1.12i), and with $|Z|_{\infty}<1$, then for almost all (small) $t$ we have the estimate (from (1.12ii)

$$
\int_{\partial \Omega} d^{p}\left(\operatorname{tr}(u)(x), u(\bar{x}(x, t)) d \Sigma_{x}^{n-1} \leq C t^{p-1} \int_{\Omega_{t}^{C}}|\nabla u|_{p} d \mu .\right.
$$

Integrating this inequality yields

$$
\int_{0}^{t_{0}} \int_{\partial \Omega} d^{p}(t r(u)(x), u(\bar{x}(x, t))) d \Sigma_{x}^{n-1} d t \leq C \frac{t_{0}^{p}}{p} \int_{\Omega_{t_{0}}^{C}}|\nabla u|_{p} d \mu .
$$

We can use the $L^{p}$-triangle inequality and the estimate (1.12vi) for two Sobolev functions $u, v$ to bound

$$
\left(\int_{0}^{t_{0}} \int_{\partial \Omega} d^{p}(t r(u)(x), \operatorname{tr}(v)(x)) d \Sigma_{x}^{n-1} d t\right)^{\frac{1}{p}}
$$

After changing variables from $d \Sigma d t$ to $d \mu$ in the appropriate term, the resulting inequality is

$$
\begin{aligned}
\left(\int_{\partial \Omega} d^{p}(\operatorname{tr}(u), \operatorname{tr}(v)) d \Sigma\right)^{\frac{1}{p}} \leq C t_{0}^{1-\frac{1}{p}} & \left.\left(\int_{\Omega_{t_{0}}^{C}}|\nabla u|_{p} d \mu\right)^{\frac{1}{p}}+\left(\int_{\Omega_{t_{0}}^{C}}|\nabla v|_{p} d \mu\right)^{\frac{1}{p}}\right) \\
& +C t_{0}^{-\frac{1}{p}}\left(\int_{\Omega_{t_{0}}} d^{p}(u, v) d \mu\right)^{\frac{1}{p}} .
\end{aligned}
$$

This inequality shows that if the sequence $\left\{u_{i}\right\}$ converges to $u$ in $L^{p}(\Omega, X)$, and if the $\left\{u_{i}\right\}$ have uniformly bounded energies, then the traces converge to the trace of $u$. Specifically, we know from semicontinuity that the energy of $u$ is bounded by those of the $u_{i}$, so the first term on the right-side of (1.12vii) can be made arbitrarily small (for the function choices $u=u, v=u_{i}$ ) by 
choosing $t_{0}$ small. Once $t_{0}$ is chosen, the second term can be made small by choosing $i$ large. Next we prove our characterization of equal-trace functions. Using (1.12vi) for the functions $u$ and $v$, we make another choice in the $L^{p_{-}}$ triangle inequality (and change variables as above) to estimate the left-hand side below:

(1.12viii)

$$
\begin{aligned}
& \left(\int_{\Omega_{\rho t_{0}}^{C}} d^{p}(u, v) d \mu\right)^{\frac{1}{p}} \leq C_{p} t_{0}\left(\left(\int_{\Omega_{t_{0}}^{C}}|\nabla u|_{p} d \mu\right)^{\frac{1}{p}}+\left(\int_{\Omega_{t_{0}}}|\nabla u|_{p} d \mu\right)^{\frac{1}{p}}\right) \\
& +C t_{0}^{\frac{1}{p}}\left(\int_{\partial \Omega} d^{p}(\operatorname{tr}(u), \operatorname{tr}(v)) d \Sigma\right)^{\frac{1}{p}} .
\end{aligned}
$$

If $\operatorname{tr}(u)=\operatorname{tr}(v)$, then (1.12viii) implies that

$$
\lim _{t \rightarrow 0} t^{-p} \int_{\Omega_{t}^{C}} d^{p}(u(y), v(y)) d \mu(y)=0 .
$$

Write $h(y)$ for the function $d(u(y), v(y))$. Define the cut-off function $\eta_{t}(y)$ to be identically one inside $\Omega_{t}$, to be zero in the complement of $\Omega_{\frac{t}{2}}$, and to linearly interpolate 0 and 1 on the annulus of points which are between distance $\frac{t}{2}$ and $t$ from $\partial \Omega$. It is clear that $\eta_{t} h \rightarrow h$ in $L^{p}(\Omega, \mathbb{R})$, as $t \rightarrow 0$. Also,

$$
\left(\int_{\Omega}\left|\nabla\left(\eta_{t} h\right)-\nabla h\right|^{p} d \mu\right)^{\frac{1}{p}} \leq\left(\int_{\Omega} h^{p}\left|\nabla \eta_{t}\right|^{p} d \mu\right)^{\frac{1}{p}}+\left(\int_{\Omega}\left(\eta_{t}-1\right)^{p}|\nabla h|^{p} d \mu\right)^{\frac{1}{p}} .
$$

From (1.12ix) we see that as $t \rightarrow 0$ the first term on the right-side of $(1.12 \mathrm{x})$ approaches zero, and it is clear that the second one also does. Thus the functions $\eta_{t} h$ converge to $h$ in the $W^{1, p}$ norm. Since each $\eta_{t} h$ has trace zero, we deduce (by the first part of our theorem) that $h$ does too. (In fact, this argument shows (in case $u$ is a real-valued function with zero trace and $v \equiv 0$ ), that for Lipschitz domains the set of functions with zero trace are exactly the space $W_{0}^{1, p}(\Omega, \mathbb{R})$, i.e. the closure under the Sobolev norm of $C_{0}^{\infty}(\Omega)$. This fact is well-known of course.)

We must now show the converse statement, that if the trace of $h$ above is zero, then $\operatorname{tr}(u)=\operatorname{tr}(v)$. We may deduce from (1.12vi) with $u=h$, that since 
$\operatorname{tr}(h)=0$

$$
\int_{\Omega_{\rho t_{0}}^{C}} h^{p}(y) d \mu(y) \leq C \frac{t_{0}^{p}}{p} \int_{\Omega_{t_{0}}^{C}}|\nabla h|^{p} d \mu .
$$

Using this estimate in the right-hand side of (1.12vii), and letting $t_{0}$ approach zero, we conclude that $\operatorname{tr}(u)=\operatorname{tr}(v)$.

Theorem 1.12.3. Let $\Omega$ be a Lipschitz Riemannian domain which is itself a disjoint union of Lipschitz subdomains $\Omega_{1}, \Omega_{2}$ and the Lipschitz boundary $\partial \Omega_{1} \cap \partial \Omega_{2}$. Let $1<p<\infty$, and $u_{i} \in W^{1, p}\left(\Omega_{i}, X\right)$ for $i=1,2$. Suppose that the trace functions $u_{1}=u_{2}$ on $\partial \Omega_{1} \cap \partial \Omega_{2}$. The the map $u$ defined by

$$
u(x)=u_{i}(x) \quad \text { if } x \in \Omega_{i}
$$

is a finite energy map, and

$$
\int_{\Omega}|\nabla u|_{p} d \mu=\int_{\Omega_{1}}\left|\nabla u_{1}\right|_{p} d \mu+\int_{\Omega_{2}}\left|\nabla u_{2}\right|_{p} d \mu .
$$

Proof. We have restricted to the case $p>1$ in our theorem only for reasons of technical simplicity. In this case, for example, it suffices to show that $u$ is a finite-energy map, since then its energy density will agree with $u_{i}$ 's energy density in $\Omega_{i}$, and the absolute continuity of the energy density with respect to Lebesgue measure immediately implies the additivity of total energy claimed in the theorem. Let $Z$ be a transverse vector field defined in a neighborhood of $\partial \Omega_{1} \cap \partial \Omega_{2}$, satisfying (1.12i), and pointing into $\Omega_{2}$. Because of our Lipschitz hypothesis, $\partial \Omega_{1} \cap \partial \Omega_{2}$ is covered by a finite number of (relatively) open subsets $\Gamma$ having the property that each $\Gamma$ is the bi-Lipschitz image of a map $\phi$ from a radius $r$ ball in $\mathbb{R}^{n-1}$. We will normalize (by scaling) to the case

$$
\phi: B^{n-1}(0,1) \rightarrow \Gamma
$$

Then for $t_{0}$ small we use the flow induced by $Z$ to define a bi-Lipschitz map $\psi$ by

$$
\psi(y, t)=\bar{x}(\phi(y), t) \quad(y, t) \in B^{n-1}(0,1) \times\left(-t_{0}, t_{0}\right)
$$

Define the $\operatorname{map}(\mathrm{s}) v$ by $v=u \circ \psi$. Then it follows that $v=v_{2}$ is a finite energy map from $B \times\left(0, t_{0}\right)$ and $v=v_{1}$ is a finite energy map from $B \times\left(-t_{0}, 0\right)$ (because finite-energy maps are preserved under bi-Lipschitz composition). 
Furthermore, using the vector field $Z$ in $\Omega$ and $\partial_{t}$ in $B \times\left(t_{0}, t_{0}\right)$, we see that the two traces of $v$ on $B^{n-1}$ agree. If we can show that $v$ is a finite-energy map on $B \times\left(-t_{0}, t_{0}\right)$, then it will follow that $u$ is a finite-energy map in a neighborhood of $\Gamma$, and our theorem will follow by the remarks above.

Pick a (unit-) direction $\omega$ which is transverse to $\partial_{t} \equiv \partial_{1}$, and orient it so that $\omega^{1}>0$. Pick a representative for $v$ so that $v$ is Hölder continuous on almost all lines parallel to $\omega$. This is possible by Lemma 1.9 .2 and the fact that the two traces of $v$ agree on $B^{n-1}$. Parameterize the $\omega$-direction lines by $y \in B^{n-1} \times\{0\}$. We claim that for each y on which the $\omega$-line is continuous, it has finite energy, and that this energy is the sum of the directional energies corresponding to $v_{1}$ and $v_{2}$. To see why this is so, we appeal to Lemma 1.9.2 in the one-variable case, i.e. $\Pi$ is a point, and we have two finite-energy (hence Hölder continuous) maps $w_{1}:\left(-t_{0}, 0\right) \rightarrow X$ and $w_{2}:\left(-t_{0}, 0\right) \rightarrow X$ which have common (trace) value at $t=0$. We let $w$ be the resulting continuous map and wish to deduce that it has finite energy. We apply the estimate (1.9viii) and the triangle inequality to deduce that for $t<0<t+\varepsilon$,

$$
\begin{aligned}
d^{p}(w(t), w(t+\varepsilon)) & \leq 2^{p}\left(d^{p}(w(t), w(0))+d^{p}(w(0), w(t+\varepsilon))\right. \\
& \leq 2^{p} \varepsilon^{p-1}\left(\int_{t}^{0}\left|\nabla w_{1}\right|_{p} d s+\int_{0}^{t+\varepsilon}\left|\nabla w_{2}\right|_{p} d s\right) .
\end{aligned}
$$

Of course, if $t$ and $t+\varepsilon$ both lie on one side of 0 , then the estimate corresponding to $(1.12 \mathrm{xi})$ also holds, in fact without the factor of $2^{p}$. If we integrate (1.12xi) (and the corresponding estimate) between $t_{1}<0<t_{2}$, and then use Fubini's Theorem we get

$$
\int_{t_{1}}^{t_{2}} d^{p}(w(t), w(t+\varepsilon)) d t \leq 2^{p} \varepsilon^{p}\left(\int_{t_{1}}^{0}\left|\nabla w_{1}\right|_{p} d t+\int_{0}^{t_{2}+\varepsilon}\left|\nabla w_{2}\right|_{p} d t\right)
$$

i.e.

$$
\int_{t_{1}}^{t_{2}} e_{\varepsilon}(t) d t \leq 2^{p}\left(\int_{t_{1}}^{0}\left|\nabla w_{1}\right|_{p} d t+\int_{0}^{t_{2}+\varepsilon}\left|\nabla w_{2}\right|_{p} d t\right) .
$$

This last inequality shows that the $\varepsilon$-energy contribution near $t=0$ is arbitrarily small (depending on $t_{1}, t_{2}$ ), so we conclude that $w$ is a finite energy map, and that its energy is the sum of the energies of $w_{1}$ and $w_{2}$. Thus we 
have shown that for almost all $y \in B^{n-1} \times\{0\}$ the direction- $\omega$ line has finite energy, given by the sum of its two component energies. We immediately conclude (from the converse statement in Lemma 1.9.1) that our map $v$ has finite energy in the direction $\omega$, equal to the sum of its two component energies. By integrating with respect to all transverse directions (i.e. almost all directions), we see that our map $v$ satisfies

$$
\int{ }^{\omega} E d \sigma(\omega)<\infty
$$

Using the same reasoning as in the converse statement of 1.9.1, it is an easy consequence that $v \in W^{1, p}\left(B^{n-1} \times\left(-t_{0}, t_{0}\right), X\right)$. Thus Theorem 1.12.3 is proven.

1.13. Precompactness. The precompactness theorem presented here is not needed for the harmonic map theory of $\S 2$, but since it is a natural part of Sobolev theory and can be proven quickly using the results we have already developed, we include it in this chapter.

Theorem 1.13. Let $(\Omega, g)$ be a Riemannian domain, $(X, d)$ a locally compact, complete metric space, and $1 \leq p<\infty$. Let $\left\{u_{i}\right\} \subset W^{1, p}(\Omega, X)$ if $p>1$ $\left(\left\{u_{i}\right\} \subset B V(\Omega, X)\right.$ if $\left.p=1\right)$ satisfy

$$
\int_{\Omega} d^{p}(u(x), Q) d \mu(x)+E^{u_{i}} \leq C .
$$

(Here $Q$ is a fixed point in $X, C$ is a fixed constant, and $E^{u_{i}}$ is the total energy of the map $u_{i}$.) Then a subsequence of $\left\{u_{i}\right\}$ converges in $L^{p}(\Omega, X)$ to a finite energy map $u$.

Proof. The definition of locally compact which we use is that every closed ball of finite radius is compact. For $j=0$ we define $Q_{j 1}=Q$. For each $j \in \mathbb{N}$ we cover $B(Q, j)$ with a finite number of points

$$
\left\{Q_{j r}\right\}_{r=1, \ldots, N_{j}}
$$

so that every $P \in B(Q, j)$ is within $\frac{1}{j}$ of one of these points. The real-valued functions

$$
d\left(u_{i}(x), Q_{j r}\right)
$$


have bounded Sobolev (or BV) norm. (The $L^{p}$ components of the norm are bounded depending on $j$, but the energy components are uniformly bounded, by Corollary 1.6.3. Thus by the standard precompactness theorems for BV and Sobolev functions, and by Cantor diagonalization, we extract a subsequence (which we also denote by $\left\{u_{i}\right\}$ ), so that for each fixed $(j, r)$ there is an $L^{p}$ function $d_{j r}$ with

$$
d\left(u_{i}(x), Q_{j r}\right) \rightarrow d_{j r}(x)
$$

in $L^{p}(\Omega, \mathbb{R})$. By choice of subsequence we also assume that a.e.x,

$$
d\left(u_{i}(x), Q_{j r}\right) \rightarrow d_{j r}(x)<\infty
$$

for all $(j, r)$. We claim that for such an $x$ there is a unique point $u(x) \in X$ with

$$
d\left(u(x), Q_{j r}\right)=d_{j r}(x)
$$

for all $(j, r)$. To see why this is so, fix an $x$ as above and any $j$ with $j>d_{01}(x)$. Then pick $i_{0}$ so that $i \geq i_{0}$ implies

$$
\begin{aligned}
\left|d\left(u_{i}(x), Q_{j r}\right)-d_{j r}(x)\right| & <\frac{1}{j} \quad r=1, \ldots, N_{j} . \\
d\left(u_{i}(x), Q\right) & <j .
\end{aligned}
$$

By the method in which the points $\left\{Q_{j r}\right\}$ were constructed, there is an $r=$ $r(x, j)$ for which

From the triangle inequality

$$
d\left(u_{i_{0}}(x), Q_{j r(x, j)}\right)<\frac{1}{j} .
$$

$$
d\left(u_{i}(x), Q_{j r(x, j)}\right)<\frac{3}{j} \quad i \geq i_{0} .
$$

This estimate implies that the sequence of points $\left\{Q_{j r(x, j)}\right\}$ is Cauchy and that $u(x)$ is its limit.

The function $u$ is clearly measurable (with separable range), and by construction

$$
u_{i}(x) \rightarrow u(x) \quad \text { a.e. } x
$$

By Fatou's Lemma

$$
\int_{\Omega} d^{p}(u(x), Q) d \mu(x) \leq \liminf _{i \rightarrow \infty} \int_{\Omega} d^{p}\left(u_{i}(x), Q\right) d \mu(x) \leq C,
$$


so $u \in L^{p}(\Omega, X)$. By lower semicontinuity (Theorem 1.6.1) $u$ is a finite energy map, with $E^{u} \leq C$. By the triangle inequality

$$
d^{p}\left(u_{i}(x), u(x)\right) \leq 2^{p} d^{p}\left(u_{i}(x), Q\right)+2^{p} d^{p}(u(x), Q) .
$$

By construction the sequence on the right is convergent in $L^{1}(\Omega, \mathbb{R})$ to the function $2^{p+1} d^{p}(u(x), Q)$. By a well-known extension of the Lebesgue Dominated Convergence Theorem it follows that

$$
\int_{\Omega} d^{p}\left(u_{i}(x), u(x)\right) d \mu \rightarrow 0,
$$

so $u_{i} \rightarrow u$ in $L^{p}(\Omega, X)$.

\section{Harmonic Maps into Non-Positively Curved Metric Spaces}

In $\S 2.1$ we recall what it means for a metric space to be non-positively curved (NPC). The definition is made using triangle comparisons to Euclidean space, and generalizes the Riemannian notion of non-positive sectional curvature (in the case of simply-connected manifolds). We recall some useful quadrilateral comparison inequalities which appear in a paper of Y.G. Reshetnyak [Re]. We combine these consequences of the NPC definition with the $W^{1,2}(\Omega, X)$ Sobolev-Space theory from Chapter 1 in order to study harmonic map questions. In $\S 2.2$ we study the Dirichlet problem, i.e. the problem of finding a $W^{1,2}(\Omega, X)$ map with given trace which is stationary (among $W^{1,2}(\Omega, X)$ maps having the same trace) for the $p=2$ Sobolev energy. We show that there is a unique such solution, and its energy is the infimum of all admissible-function energies. In fact, the NPC hypothesis implies that the energy functional is appropriately convex with respect to natural geodesic homotopies of finiteenergy maps. This convexity allows one to show that a minimizing sequence is Cauchy in $L^{2}(\Omega, X)$, i.e. one uses an exact generalization of Dirichlet's Principle for finding harmonic functions. As in the classical case, the convexity property also implies the uniqueness result. We find the generality, elegance, and simplicity of the proofs presented here to be an indication that we have found the proper framework for their expression. The combination of the correct definition of energy (using distances) with the distance comparison results which follow from the NPC hypothesis allows one to replace arguments 
which are customarily made with first derivative expressions with ones only involving (zero ${ }^{\text {th }}$ - order) distance inequalities. The existence theorem in $\S 2.2$ uses the completeness of the target but not any local compactness properties. This is important for the applications discussed in subsequent work, where for example, we consider targets $L^{2}(M, X)$ where $\mathrm{M}$ is a Riemannian manifold and $X$ is an NPC space. (Such targets are also NPC.)

In Chapter 1 we described how a map $u \in W^{1,2}(\Omega, X)$ induces integrable directional-energy functions $\left|u_{*}(Z)\right|^{2}(x)$ for fixed vector fields $Z \in \Gamma T(\bar{\Omega})$. In $\S 2.3$ we show that it is a consequence of the NPC hypothesis that these directional-energy functions satisfy a parallelogram law, i.e.

$$
\left|u_{*}(Z+W)\right|^{2}+\left|u_{*}(Z-W)\right|^{2}=2\left|u_{*}(Z)\right|^{2}+2\left|u_{*}(W)\right|^{2} .
$$

Thus there is a non-negative integrable tensor $\pi$, which generalizes the notion of the pull-back metric $u^{*} h$ for maps to Riemannian targets $(N, h)$, and so that the $p=2$ Sobolev energy-density function $|\nabla u|^{2}$ is given by

$$
|\nabla u|^{2}=g^{i j} \pi_{i j}
$$

in local coordinates. The inner product $\pi$ plays an important role in understanding the structure of harmonic maps to NPC spaces, and in applications to rigidity theory.

One can follow the approach of [GS] in order to prove the interior Lipschitz continuity of harmonic maps into NPC spaces, generalizing various arguments when necessary. In $\S 2.4$ we choose instead to deduce this regularity by proving a (weak) form of the classical Bochner inequality for $\Delta|\nabla u|^{2}$, implying that $|\nabla u|^{2}$ is essentially subharmonic. In the case of a harmonic function, this inequality can be obtained by a finite differencing technique applied to the energy integral. (Of course it follows formally just by computing $\Delta|\nabla u|^{2}$.) This technique involves retracting the solution $u$ towards translates of itself, estimating the change in energy and noting that it must be non-negative. These ideas generalize to the NPC setting, and imply the same Bochner inequality.

In $\S 2.5$ we recall how to construct centers of mass for maps to NPC spaces. We use the quadrilateral comparisons of [Re] (discussed in (2.1)) in order to derive quantitative estimates for the distance between the centers of mass of 
different maps (with respect to different weightings). By integrating these estimates one can bound the energy of averages of maps in terms of averages of the energies. In $\S 2.6$ we study the equivariant harmonic map problem. We indicate how to construct initial finite-energy maps for certain configurations, and how to combine the solutions to Dirichlet problems with the averaging techniques in $\S 2.5$, in order to produce minimizing sequences with uniform (local) Lipschitz continuity control. This approach is analogous to, but technically different from, the Perron method of finding harmonic functions. We conclude the paper in $\S 2.7$, where we indicate how to solve the homotopy problem for harmonic maps to spaces with NPC universal covers; we present the natural generalization of the classical results due to Eells and Sampson [ES].

2.1. Non-positively curved metric spaces. A complete metric space $(X, d)$ is said to be non-positively curved (NPC) if the following two conditions are satisfied:

(i) $(X, d)$ is a length space. That is, for any two points $P, Q$ in $X$, the distance $d(P, Q)$ is realized as the length of a rectifiable curve connecting $P$ to $Q$. (We call such distance-realizing curves geodesics.)

(ii) For any three points $P, Q, R$ in $X$ and choices of geodesics $\gamma_{P, Q}$ (of length $r$ ), $\gamma_{Q, R}$ (of length $p$ ), and $\gamma_{R, P}$ (of length $q$ ) connecting the respective points, the following comparison property is to hold: For any $0<\lambda<1$ write $Q_{\lambda}$ for the point on $\gamma_{Q, R}$ which a fraction $\lambda$ of the distance from $Q$ to $R$. That is,

$$
d\left(Q_{\lambda}, Q\right)=\lambda p, d\left(Q_{\lambda}, R\right)=(1-\lambda) p .
$$

On the (possibly degenerate) Euclidean triangle of side lengths $p, q, r$ and opposite vertices $\bar{P}, \bar{Q}, \bar{R}$, there is a corresponding point

$$
\bar{Q}_{\lambda}=\bar{Q}+\lambda(\bar{R}-\bar{Q}) .
$$

The NPC hypothesis is that the metric distance $d\left(P, Q_{\lambda}\right)$ (from $Q_{\lambda}$ to its opposite vertex) is bounded above by the Euclidean distance $\left|\bar{P}-\bar{Q}_{\lambda}\right|$. This inequality can be written precisely as

$$
d^{2}\left(P, Q_{\lambda}\right) \leq(1-\lambda) d^{2}(P, Q)+\lambda d^{2}(P, R)-\lambda(1-\lambda) d^{2}(Q, R) .
$$


Using successive subdivision one sees that knowing the comparison property just in the case $\lambda=\frac{1}{2}$ suffices to prove it for all $0<\lambda<1$.

It is any easy consequence of property (ii) above that geodesics in an NPCspace are unique. Indeed, if $\gamma_{1}$ and $\gamma_{2}$ are two geodesic paths from $Q$ to $R$, pick $P$ to be a point on $\gamma_{1}$, say at a fraction $\mu(0<\mu<1)$ of the way from $Q$ to $R$. Take $\lambda=\mu$ and construct $Q_{\mu}$ (on the geodesic $\gamma_{2}$ ) as above. By construction the comparison Euclidean triangle must degenerate to a line segment, so that $\bar{P}=\bar{Q}_{\mu}$, i.e. $d\left(\bar{P}, \bar{Q}_{\mu}\right)=0$. The comparison property (ii) implies therefore that $d\left(P, Q_{\mu}\right)=0$ as well. Since $\mu$ is arbitrary, we conclude that the geodesics $\gamma_{1}$ and $\gamma_{2}$ coincide. At least in the case that $X$ is locally compact, it is an easy consequence of geodesic uniqueness that an NPC space $X$ must be simply connected. Conversely, it is a well-known fact (proved with Jacobi Field analysis) that any complete, simply connected Riemannian manifold having non-positive sectional curvature is an example of an NPC space. (One defines the distance between two points to be the infimum of curve lengths for paths connecting the points.) There are many examples of non-Riemannian spaces which are NPC, e.g. trees, Euclidean Buildings, Hilbert Spaces, and other infinite dimensional symmetric spaces. Also, if $X$ is NPC and if $(M, g)$ is a finite-volume Riemannian manifold, then the space $L^{2}(M, X)$ is also NPC.

A useful consequence of the NPC hypothesis is a quadrilateral comparison property, which we now describe. These results appear as lemmas in a substantially more general theorem due to Reshetnyak [Re], and we include their proofs here for completeness.

Let $\{P, Q, R, S\}$ be an ordered sequence of four points in $(X, d)$. We say that $\{P, Q, R, S\}$ is sub-embeddable into $\mathbb{R}^{2}$ if there exists an ordered sequence

$$
\{\bar{P}, \bar{Q}, \bar{R}, \bar{S}\} \subset \mathbb{R}^{2}
$$

so that

$$
\begin{aligned}
& d(P, Q)=|\bar{P}-\bar{Q}|, \quad d(Q, R)=|\bar{Q}-\bar{R}| \\
& d(R, S)=|\bar{R}-\bar{S}|, \quad d(S, P)=|\bar{S}-\bar{P}| \\
& d(P, R) \leq|\bar{P}-\bar{R}|, \quad d(Q, S) \leq|\bar{Q}-\bar{S}|
\end{aligned}
$$


In the above construction we call $\{\bar{P}, \bar{Q}, \bar{R}, \bar{S}\}$ a sub-embedding for $\{P, Q, R, S\}$. (If any collection of points has a corresponding collection in $\mathbb{R}^{2}$ for which all pairwise distances between corresponding points are equal, we call the Euclidean collection an embedding of the metric collection.) If a metric space has the property that every ordered sequence of four points can be sub-embedded into $\mathbb{R}^{2}$, we say that $(X, d)$ satisfies the sub-embedding property.

Theorem 2.1.1. [Re] A length space is NPC if and only if it satisfies the subembedding property above. In fact, if $(X, d)$ is NPC then one may always pick a sub-embedding $\{\bar{P}, \bar{Q}, \bar{R}, \bar{S}\}$ for $\{P, Q, R, S\}$ so that the Euclidean sequence forms the consecutive vertices of a convex quadrilateral.

Proof. Assume $(X, d)$ satisfies the sub-embedding property. Let $\{P, Q, R\} \subset$ $X$ and $0<\lambda<1$. Construct $Q_{\lambda}$ as in the discussion (ii) above. Then the sequence $\left\{P, Q, Q_{\lambda}, R\right\}$ has a sub-embedding $\left\{\bar{P}, \bar{Q}, \bar{Q}_{\lambda}, \bar{R}\right\} \subset \mathbb{R}^{2}$. From (2.1i) and (2.1iii) we deduce that $\bar{Q}_{\lambda}$ lies on the line segment connecting $\bar{Q}$ to $\bar{R}$, i.e.

$$
\bar{Q}_{\lambda}=\bar{Q}+\lambda(\bar{R}-\bar{Q})
$$

Thus the sub-embedding hypothesis (2.1iii) that $d\left(P, Q_{\lambda}\right) \leq\left|\bar{P}-\bar{Q}_{\lambda}\right|$ is exactly the requirement for the triangle comparison property (ii) to hold. Thus $(X, d)$ is NPC.

Conversely, let $(X, d)$ be NPC. Let $\{P, Q, R, S\} \subset X$ be given. Construct Euclidean embeddings $\{\bar{P}, \bar{Q}, \bar{S}\}$ and $\{\bar{Q}, \bar{R}, \bar{S}\}$ for the points $\{P, Q, S\}$ and $\{Q, R, S\}$ respectively. We may construct these embeddings so that the resulting triangles share the edge $\overline{Q S}$, and so that $\bar{P}$ and $\bar{R}$ lie on opposite sides of the edge.

Case $I$. The quadrilateral with consecutive vertices $\{\bar{P}, \bar{Q}, \bar{R}, \bar{S}\}$ is convex. In this case we claim that $d(P, R) \leq|\bar{P}-\bar{R}|$ so that we have a suitable subembedding. To verify this claim consider the diagonal $\overline{P R} \subset \mathbb{R}^{2}$. It intersects $\overline{Q S}$ at a point

$$
\bar{Q}_{\lambda}=\bar{Q}+\lambda(\bar{S}-\bar{Q})
$$


Consider the corresponding point $Q_{\lambda}$ on the geodesic (in $X$ ) connecting $Q$ to $S$. Then from the triangle inequality and the NPC hypothesis we have

$$
\begin{aligned}
d(P, Q) & \leq d\left(P, Q_{\lambda}\right)+d\left(Q_{\lambda}, R\right) \\
& \leq\left|\bar{P}-\bar{Q}_{\lambda}\right|+\left|\bar{Q}_{\lambda}-\bar{R}\right| \\
& =|\bar{P}-\bar{R}| .
\end{aligned}
$$

This proves the claim.

Case II. The quadrilateral with consecutive vertices $\{\bar{P}, \bar{Q}, \bar{R}, \bar{S}\}$ is not convex. Because the interior angles of a Euclidean quadrilateral sum to $2 \pi$, exactly one of the interior angles $\angle \bar{P}, \bar{Q}, \bar{R}, \angle \bar{R}, \bar{S}, \bar{P}$ is greater than $\pi$. We assume (by relettering) that $\angle \bar{P}, \bar{Q}, \bar{R}>\pi$. We orient our points so that $\bar{R}=(0,0)$, $\bar{P}=(0, a), a>0$, and so that $\bar{Q}$ and $\bar{S}$ lie to the right of the $y$-axis, (with $\bar{S}$ farther to the right).

Case IIa. $d(P, R) \leq|\bar{P}-\bar{R}|$. In this case our points are a sub-embedding. If we reflect $\bar{Q}$ across the $y$-axis we increase the distance $|\bar{Q}-\bar{S}|$ and leave the other five pairwise distances unchanged. Thus we create a convex sub-embedding. Case IIb. $d(P, R)>|\bar{P}-\bar{R}|$. In this case we do not yet have a sub-embedding. If we lift $\bar{P}$ (increase $a$ ), keeping $\bar{R}=(0,0)$ fixed, then $\bar{Q}, \bar{S}$ must move uniquely if we require

$$
\begin{array}{ll}
d(\bar{P}, \bar{Q}), & d(\bar{Q}, \bar{R}), \\
d(\bar{P}, \bar{S}), & d(\bar{S}, \bar{R})
\end{array}
$$

to remain fixed. Using elementary geometry one can check that the diagonal distance $|\bar{Q}-\bar{S}|$ increases as $a$ increases. (One shows that the interior angles $\angle \bar{Q}, \bar{R}, \bar{S}, \angle \bar{S}, \bar{P}, \bar{Q}$ are increasing with respect to $a$, by studying the rates of change of the other two interior angles of the quadrilateral determined by the sequence $\{\bar{P}, \bar{Q}, \bar{R}, \bar{S}\}$.) We continue increasing $a$ until $d(P, R)=|\bar{P}-\bar{R}|$. This must happen by the time $\bar{Q}$ hits the $y$-axis, since

$$
d(P, R) \leq d(P, Q)+d(Q, R)=|\bar{P}-\bar{Q}|+|\bar{Q}-\bar{R}|=|\bar{P}-\bar{R}|
$$

in this case. Thus one obtains a sub-embedding for some $0<a \leq d(P, R)$, and by reflection as in Case IIa one may assume that the points $\{\bar{P}, \bar{Q}, \bar{R}, \bar{S}\}$ are consecutive vertices of a convex quadrilateral. 
Theorem 2.1.2. [Re] Let $(X, d)$ be an NPC space. Let $\{P, Q, R, S\} \subset X$ be an ordered sequence and let $\{\bar{P}, \bar{Q}, \bar{R}, \bar{S}\} \subset \mathbb{R}^{2}$ be a sub-embedding. Let $0 \leq \lambda, \mu \leq 1$ be given. Define $P_{\lambda}$ to be the point which is the fraction $\lambda$ of the way from $P$ to $S$ (on the geodesic $\gamma_{P, S}$ ). Let $Q_{\mu}$ be the point which is the fraction $\mu$ of the way from $Q$ to $R$ (along the opposite geodesic $\gamma_{Q, R}$. Construct the corresponding Euclidean points

$$
\bar{P}_{\lambda}=(1-\lambda) \bar{P}+\lambda \bar{S}, \bar{Q}_{\mu}=(1-\mu) \bar{Q}+\mu \bar{R} .
$$

Then

$$
d\left(P_{\lambda}, Q_{\mu}\right) \leq\left|\bar{P}_{\lambda}-\bar{Q}_{\mu}\right|
$$

Proof. This estimate follows from repeated use of the triangle comparison property. As a preliminary step we claim that

$$
d\left(P, Q_{\mu}\right) \leq\left|\bar{P}-\bar{Q}_{\mu}\right| .
$$

In fact, we can estimate the relative lengths, using the appropriate Euclidean identity and the triangle comparison property (2.1ii):

$$
\begin{aligned}
\left|\bar{P}-\bar{Q}_{\mu}\right|^{2} & =(1-\mu)|\bar{P}-\bar{Q}|^{2}+\mu|\bar{P}-\bar{R}|^{2}-\mu(1-\mu)|\bar{Q}-\bar{R}|^{2} \\
& \geq(1-\mu) d^{2}(P, Q)+\mu d^{2}(P, R)-\mu(1-\mu) d^{2}(Q, R) \\
& \geq d^{2}\left(P, Q_{\mu}\right) .
\end{aligned}
$$

Analogously,

$$
d\left(S, Q_{\mu}\right) \leq\left|\bar{S}-\bar{Q}_{\mu}\right|
$$

Using the comparison property a final time we estimate

$$
\begin{aligned}
\left|\bar{P}_{\lambda}-\bar{Q}_{\mu}\right|^{2} & =(1-\lambda)\left|\bar{P}-\bar{Q}_{\mu}\right|^{2}+\lambda\left|\bar{S}-\bar{Q}_{\mu}\right|^{2}-\lambda(1-\lambda)|\bar{P}-\bar{S}|^{2} \\
& \geq(1-\lambda) d^{2}\left(P, Q_{\mu}\right)+\lambda d^{2}\left(S, Q_{\mu}\right)-\lambda(1-\lambda) d^{2}(P, S) \\
& \geq d^{2}\left(P_{\lambda}, Q_{\mu}\right) . \quad \square
\end{aligned}
$$

Corollary 2.1.3. Abbreviate the distance function $d(T, U)$ by $d_{T U}$. For an ordered sequence $\{P, Q, R, S\} \subset X$, define the geodesic interpolation points 
$P_{\lambda}, Q_{\mu}$ as in Theorem 2.1.2. Then for any $0 \leq \alpha, t \leq 1$ the following estimates hold.

$$
\begin{aligned}
d^{2}\left(P_{t}, Q_{t}\right) \leq & (1-t) d_{P Q}^{2}+t d_{R S}^{2} \\
& -t(1-t)\left(\alpha\left(d_{S P}-d_{Q R}\right)^{2}+(1-\alpha)\left(d_{R S}-d_{P Q}\right)^{2}\right)
\end{aligned}
$$

$$
\begin{aligned}
d^{2}\left(Q_{t}, P\right)+d^{2}\left(Q_{1-t}, S\right) \leq & d_{P Q}^{2}+d_{R S}^{2}+t\left(d_{S P}^{2}-d_{Q R}^{2}\right)+2 t^{2} d_{Q R}^{2} \\
& -t\left(\alpha\left(d_{S P}-d_{Q R}\right)^{2}+(1-\alpha)\left(d_{R S}-d_{P Q}\right)^{2}\right) .
\end{aligned}
$$

In case $t=1$ in (2.1v) we deduce the parallelogram inequality:

$$
\begin{aligned}
d_{P R}^{2}+d_{Q S}^{2} \leq & d_{P Q}^{2}+d_{Q R}^{2}+d_{R S}^{2}+d_{S P}^{2} \\
& -\alpha\left(d_{S P}-d_{Q R}\right)^{2}-(1-\alpha)\left(d_{R S}-d_{P Q}\right)^{2} .
\end{aligned}
$$

Proof. Given $\{P, Q, R, S\} \subset X$ we pick a subembedding $\{\bar{P}, \bar{Q}, \bar{R}, \bar{S}\} \subset \mathbb{R}^{2}$, and take $A, B, C, D$ to be the oriented vectors pointing to consecutive vertices of the Euclidean quadrilateral, i.e.

$$
\begin{array}{ll}
A=\bar{Q}-\bar{P} & B=\bar{R}-\bar{Q} \\
C=\bar{S}-\bar{R} & D=\bar{P}-\bar{S} .
\end{array}
$$

We have the Euclidean identity

$$
\begin{aligned}
\left|\bar{P}_{t}-\bar{Q}_{t}\right|^{2} & =\frac{1}{2}|t(B+D)+A|^{2}+\frac{1}{2}|(1-t)(B+D)+C|^{2} \\
& =t|C|^{2}+(1-t)|A|^{2}-t(1-t)|B+D|^{2} .
\end{aligned}
$$

We have omitted some intermediate computations in the above identity, in which one makes repeated use of the fact that $A+B+C+D=0$. Since $|A+C|=|B+D|$ dominates both ||$C|-| A||$ and ||$D|-| B||$, the sub-embedding hypothesis, Theorem 2.1.2 and (2.1vii) combine to imply (2.1iv).

Another Euclidean identity (for $A+B+C+D=0$ ) is

$$
\begin{aligned}
|t B+A|^{2}+|t B+C|^{2} & =|A|^{2}+|C|^{2}+t^{2}|B|^{2} \\
& +t\left(|D|^{2}-|B|^{2}\right)-t|B+D|^{2} .
\end{aligned}
$$

It yields $(2.1 \mathrm{v})$, which then yields $(2.1 \mathrm{vi})$ by setting $t=1$. 
2.2. The solution to the Dirichlet Problem. To motivate the general existence and uniqueness theorem proved below we first recall Dirichlet's variational principle in the classical case where $X=\mathbb{R}$. (The general proof will follow the same outline.) Let $(\Omega, g)$ be a Riemannian domain. Given a map $\phi \in W^{1,2}(\Omega, \mathbb{R})$ we consider the closed convex subset

$$
W_{\phi}^{1,2}(\Omega, \mathbb{R})=\left\{u \in W^{1,2}(\Omega, \mathbb{R}) \mid u-\phi \in W_{0}^{1,2}(\Omega, \mathbb{R})\right\} .
$$

$\left(W_{0}^{1,2}(\Omega, \mathbb{R})\right.$ is discussed in (1.12.2).) A harmonic function $u$ is one that is critical for the Dirichlet integral, with respect to small $W_{0}^{1,2}$ perturbations. In fact, there is a unique $u \in W_{\phi}^{1,2}(\Omega, \mathbb{R})$ which is critical, and it is the unique energy minimizer in that class, as the following argument shows. Define

$$
E_{0}=\inf _{v \in W_{\phi}^{1,2}(\Omega, \mathbb{R})} \int|\nabla v|^{2} .
$$

Recall the parallelogram identity for $u, v \in W^{1,2}(\Omega, \mathbb{R})$ :

$$
\int_{\Omega}\left|\nabla\left(\frac{u+v}{2}\right)\right|^{2} d \mu+\int_{\Omega}\left|\nabla\left(\frac{u-v}{2}\right)\right|^{2} d \mu=\frac{1}{2} \int_{\Omega}|\nabla u|^{2} d \mu+\frac{1}{2} \int_{\Omega}|\nabla v|^{2} d \mu .
$$

Pick a minimizing sequence $\left\{u_{i}\right\} \subset W_{\phi}^{1,2}(\Omega, \mathbb{R})$, i.e. one for which the corresponding Dirichlet integrals converge to $E_{0}$. Pick $u=u_{i}, v=u_{j}$ in (2.2i). As $i, j \rightarrow \infty$ the right side of $(2.2 \mathrm{i})$ converges to $E_{0}$. Since $\frac{u+v}{2} \in W_{\phi}^{1,2}(\Omega, \mathbb{R})$, the first term on the left-hand side of $(2.2 \mathrm{i})$ is at least $E_{0}$. We deduce that for a minimizing sequence

$$
\lim _{i, j \rightarrow \infty} \int_{\Omega}\left|\nabla\left(u_{i}-u_{j}\right)\right|^{2}=0 .
$$

But $u_{i}-u_{j} \in W_{0}^{1,2}(\Omega, \mathbb{R})$ and for $v \in W_{0}^{1,2}(\Omega, \mathbb{R})$ one has the Poincaré inequality

$$
\int_{\Omega} v^{2} d \mu \leq C(\Omega) \int_{\Omega}|\nabla v|^{2} d \mu
$$

Hence

$$
\lim _{i, j \rightarrow 0} \int_{\Omega}\left(u_{i}-u_{j}\right)^{2} d \mu=0
$$

as well. Thus $\left\{u_{i}\right\}$ is Cauchy in the $W^{1,2}$ norm, and it converges to $u \in W_{\phi}^{1,2}$ with energy $E_{0}$. If $v$ is any other admissable function then $v_{t}=(1-t) u+t v$ 
is an admissable family and (2.2i) implies the Dirichlet integrals are a strictly convex function of $t$ (unless $u=v$ ). Since this convex function has zero derivative at $t=0$, its derivative must be non-zero at $t=1$, so no other $v \in W_{\phi}^{1,2}$ can be harmonic.

We now prove the general result:

Theorem 2.2. Let $(\Omega, g)$ be a Lipschitz Riemannian domain and let $(X, d)$ be a NPC metric space. Let $\phi \in W^{1,2}(\Omega, X)$. Define

$$
W_{\phi}^{1,2}=\left\{u \in W^{1,2}(\Omega, X) \mid \operatorname{tr}(u)=\operatorname{tr}(\phi)\right\}
$$

Then there exists a unique $u \in W_{\phi}^{1,2}$ which is stationary for the $p=2$ Sobolev energy. In fact, the energy $E^{u}=\int|\nabla u|^{2} d \mu$ of $u$ satisfies

$$
E^{u}=E_{0} \equiv \inf _{v \in W_{\phi}^{1,2}} E^{v}
$$

(Note that our definition of $E$ in this chapter is consistent with $(1.10 \mathrm{v})$, so differs from the one in the rest of Chapter 1 by a multiplicative constant.)

Proof. Let $u, v \in W^{1,2}(\Omega, X)$. Then there is a natural analog of the map $\frac{u+v}{2}$ considered above, namely we define $w(x)$ to be the midpoint of the geodesic connecting $u(x)$ to $v(x)$. It is easy to check that $w \in L^{2}(\Omega, X)$.

If $x, y \in \Omega$ we consider the sequence $\{u(y), u(x), v(x), v(y)\} \subset X$. Setting $t=\frac{1}{2}$ and $\alpha=1$ in (2.1iv) of Corollary 2.1.3 we deduce

$$
\begin{aligned}
2 d^{2}(w(x), w(y)) \leq & d^{2}(u(x), u(y))+d^{2}(v(x), v(y)) \\
& -\frac{1}{2}(d(u(y), v(y))-d(u(x), v(x)))^{2} .
\end{aligned}
$$

Multiplying (2.2iii) by $f(x)$ (where $f \geq 0$ and $f \in C_{c}(\Omega)$ ), then integrating and averaging on the subset $|x-y|<\varepsilon$ of $\Omega \times \Omega$ (as in (1.3)) we deduce first of all that $w \in W^{1,2}(\Omega, X)$. From Theorem 1.12 .2 we conclude that $w$ has trace $\phi$, so is an admissable function. We also conclude that

$$
2 \int_{\Omega} f|\nabla w|^{2} \leq \int_{\Omega} f|\nabla u|^{2}+\int_{\Omega} f|\nabla v|^{2}-\frac{1}{2} \int_{\Omega} f|\nabla d(u, v)|^{2} d \mu
$$


for any $f \in C_{c}(\Omega), f \geq 0$. (We have used Theorem 1.6.2.) Hence we have the analog of $(2.2 \mathrm{i})$ :

$$
2 E^{w} \leq E^{u}+E^{v}-\frac{1}{2} \int_{\Omega}|\nabla d(u, v)|^{2} d \mu .
$$

Now let $\left\{u_{i}\right\} \subset W_{\phi}^{1,2}$ be a minimizing sequence. From (2.2iv) we see that

$$
\lim _{i, j \rightarrow \infty} \int_{\Omega}\left|\nabla d\left(u_{i}, u_{j}\right)\right|^{2} d \mu=0 .
$$

Since $d\left(u_{i}, u_{j}\right) \in W_{0}^{1,2}(\Omega, \mathbb{R})$ (Theorem 1.12.2), the Poincaré inequality (2.2ii) holds and we deduce that $\left\{u_{i}\right\}$ converges in $L^{2}(\Omega, X)$ to a limit function $u$. By Theorem 1.12 .2 we have $u \in W_{\phi}^{1,2}$, so by semicontinuity (1.6.1) we also have $E^{u}=E_{0}$. If $v$ is any other admissable function then we may define the one parameter family of $L^{2}$ functions $u_{t}$, by defining $u_{t}(x)$ to be the point which is a fraction $t$ of the way from $u(x)$ to $v(x)$, on the connecting geodesic. From (2.1iv) with $\alpha=1$ we have

$$
\begin{aligned}
d^{2}\left(u_{t}(x), u_{t}(y)\right) & \leq(1-t) d^{2}(u(x), u(y))+t d^{2}(v(x), v(y)) \\
& -t(1-t)\left(d(u(y), v(y))-d(u(x), v(x))^{2} .\right.
\end{aligned}
$$

It follows as above that each $u_{t} \in W_{\phi}^{1,2}$, and that

$$
E^{u_{t}} \leq(1-t) E^{u}+t E^{v}-t(1-t) \int_{\Omega}|\nabla d(u, v)|^{2} .
$$

Therefore, if $u \neq v$ the function $E^{u_{t}}$ is strictly convex. Since it has a minimum at $t=0$, it is strictly increasing at $t=1$, so no other admissable $v$ can be harmonic.

2.3. The pull-back inner product $\pi$. We prove the parallelogram identity discussed in the introduction, and discuss the resulting non-negative $L^{1}$ tensor $\pi$.

Lemma 2.3.1. Let $(\Omega, g)$ be a Riemannian domain and let $X$ be an $N P C$ metric space. If $u \in W^{1,2}(\Omega, X)$, then for any $Z, W \in \Gamma(T \bar{\Omega})$ the parallelogram identity

$$
\left|u_{*}(Z+W)\right|^{2}+\left|u_{*}(Z-W)\right|^{2}=2\left|u_{*}(Z)\right|^{2}+2\left|u_{*}(W)\right|^{2}
$$


holds.

Proof. We integrate the pointwise parallelogram inequality (2.1vi) against a non-

negative $f \in C_{c}(\Omega)$ as follows. For $\varepsilon>0$ fixed, and for each $x$ (sufficiently in) the interior of $\Omega$, write

$$
\begin{gathered}
x_{1}(\varepsilon)=x+\varepsilon Z(x), \quad x_{2}(\varepsilon)=x+\varepsilon(Z+W)(x), \\
x_{3}(\varepsilon)=x+\varepsilon W(x) .
\end{gathered}
$$

Then we have:

$$
\begin{aligned}
\int_{\Omega} f(x)\{ & \frac{d^{2}\left(u(x), u\left(x_{2}(\varepsilon)\right)\right)}{\varepsilon^{2}}+\frac{\left.d^{2} u\left(x_{1}(\varepsilon)\right), u\left(x_{3}(\varepsilon)\right)\right)}{\varepsilon^{2}} \\
& -\frac{d^{2}\left(u(x), u\left(x_{1}(\varepsilon)\right)\right)}{\varepsilon^{2}}-\frac{d^{2}\left(u\left(x_{2}(\varepsilon)\right), u\left(x_{1}(\varepsilon)\right)\right.}{\varepsilon^{2}} \\
& \left.-\frac{d^{2}\left(u\left(x_{3}(\varepsilon)\right), u\left(x_{2}(\varepsilon)\right)\right)}{\varepsilon^{2}}-\frac{\left.d^{2}\left(u\left(x_{3}(\varepsilon)\right), u(x)\right)\right)}{\varepsilon^{2}}\right\} d \mu \leq 0 .
\end{aligned}
$$

We claim that as $\varepsilon \rightarrow 0$ this expression converges to the inequality

$$
\int_{\Omega} f\left(\left|u_{*}(Z+W)\right|^{2}+\left|u_{*}(Z-W)\right|^{2}-2\left|u_{*}(Z)\right|^{2}-2\left|u_{*}(W)\right|^{2}\right) d \mu \leq 0 .
$$

To see why this is true consider the second term, for example. By changing coordinates from $x$ to $y=x_{3}(\varepsilon)=x+\varepsilon W(x)$ we see that it may be rewritten as

$$
\int_{\Omega}(f(y)+o(1)) \frac{d^{2}(u(y), u(y+\varepsilon((Z-W)(y)+o(1)))}{\varepsilon^{2}}(1+C \varepsilon) d \mu(y) .
$$

Here the first $o(1)$ term depends on the modulus of continuity function

$$
\omega\left(f, \varepsilon|Z|_{\infty}\right)(y),
$$

and the second one is the difference between $Z-W$ evaluated at $x$ and $y$. It follows from Theorem 1.8.1 that as $\varepsilon \rightarrow 0$ this integral converges to

$$
\int_{\Omega} f(x)\left|u_{*}(Z-W)\right|^{2}(x) d \mu(x) .
$$

From the argument above we conclude that

$$
\left|u_{*}(Z+W)\right|^{2}+\left|u_{*}(Z-W)\right|^{2} \leq 2\left|u_{*}(Z)\right|^{2}+2\left|u_{*}(W)\right|^{2} .
$$


Applying (2.3i) to the vector fields $Z+W$ and $Z-W$ yields

$$
\left|u_{*}(2 Z)\right|^{2}+\left|u_{*}(2 W)\right|^{2} \leq 2\left|u_{*}(Z+W)\right|^{2}+2\left|u_{*}(Z-W)\right|^{2}
$$

which is exactly the reverse inequality. Thus the parallelogram identity holds.

For $Z, W \in \Gamma T(\bar{\Omega})$ we define

$$
\pi(Z, W) \equiv \frac{1}{4}\left|u_{*}(Z+W)\right|^{2}-\frac{1}{4}\left|u_{*}(Z-W)\right|^{2} .
$$

Theorem 2.3.2. The operator $\pi$ defined above,

$$
\pi: \Gamma(T \bar{\Omega}) \times \Gamma(T \bar{\Omega}) \rightarrow L^{1}(\Omega, \mathbb{R})
$$

is continuous, symmetric, bilinear, non-negative, and tensorial. Specifically,

$$
\begin{aligned}
\pi(Z, Z) & =\left|u_{*}(Z)\right|^{2} \geq 0 \\
\pi(Z, W) & =\pi(W, Z) \\
\pi(Z, h V+W) & =h \pi(Z, V)+\pi(Z, W) \quad\left(h \in C^{0,1}(\bar{\Omega})\right) .
\end{aligned}
$$

If $(\Omega, g)$ has local coordinates

$$
\left(x^{1}, x^{2}, \ldots, x^{n}\right)
$$

and corresponding tangent basis

$$
\left\{\partial_{1}, \ldots, \partial_{n}\right\}
$$

we write

$$
\pi_{i j}=\pi\left(\partial_{i}, \partial_{j}\right)
$$

Then for $Z=Z^{i} \partial_{i}$ and $W=W^{j} \partial_{j}$ we have

$$
\pi(Z, W)=\pi_{i j} Z^{i} W^{j}
$$

If $\psi: \Omega_{1} \rightarrow \Omega$ is a $C^{1,1}$ map, then writing $v=u \circ \psi$, and $\pi_{v}$ for the corresponding operator, we have the formula

$$
\left(\pi_{v}\right)_{i j}=\pi_{l m} \psi_{, i}^{l} \psi_{, j}^{m}
$$

Hence in local coordinates

$$
|\nabla u|^{2}=g^{i j} \pi_{i j}
$$


where $\left[g^{i j}\right]$ is (as usual) the inverse matrix to the Riemannian metric matrix

$$
\left[g_{i j}\right]=\left[\left\langle\partial_{i}, \partial_{j}\right\rangle\right]
$$

Proof. It is clear from the definition and Theorem 1.11 that the map $\pi$ is continuous. Abbreviating $\pi(Z, W)$ by $\langle Z, W\rangle$, and writing $\langle Z, Z\rangle=|Z|^{2}$, we see from the scaling property in Theorem 1.11 that

$$
|h Z|^{2}=|h|^{2}|Z|^{2}
$$

for any $h \in C^{0,1}(\bar{\Omega})$. In particular, $|Z|^{2}=|-Z|^{2}$. We may write the parallelogram identity (2.3.1) in the customary way:

$$
|Z+W|^{2}+|Z-W|^{2}=2|Z|^{2}+2|W|^{2} .
$$

It is well-known that the parallelogram identity is equivalent to an inner product structure, and we recall the reason why. Starting with the expression $|Z+V+W|^{2}$, one uses the parallelogram identity to express it in in terms of $|Z+V-W|^{2}$ (and squares of sums of "length" one or two), then in terms of $|Z-V-W|^{2}$, and finally in terms of $|-Z-V-W|^{2}$, i.e. $|Z+V+W|^{2}$. Symmetrizing the resulting identity (via the parallelogram law) one deduces that

$$
\begin{aligned}
|Z+V+W|^{2}= & 3\left(|Z|^{2}+|V|^{2}+|W|^{2}\right) \\
& -\left(|Z-V|^{2}+|Z-W|^{2}+|V-W|^{2}\right) .
\end{aligned}
$$

This reduction formula and definition (2.3ii) imply that

$$
\pi(Z, V+W)=\pi(Z, V)+\pi(Z, W) .
$$

By successive applications of this sum-linearity and by scaling one deduces that

$$
\pi(Z, h W)=h \pi(Z, W)
$$

for any rational $h$. By continuity of $\pi$ we deduce that (2.3ix) holds for any $h \in \mathbb{R}$. By using a partition of unity argument in $\Omega$ (as in the proof of $(1.8 \mathrm{v})$ ) we deduce that (2.3ix) holds for $h \in C^{0,1}(\bar{\Omega})$. Thus we have verified (2.3iii). 
The claim (2.3iv) follows from repeated applications of sum-linearity. To see the chain rule, we note from Theorem 1.11 that

$$
\begin{aligned}
\pi_{v}\left(\bar{\partial}_{i}, \bar{\partial}_{i}\right) & =\left|v_{*}\left(\bar{\partial}_{i}\right)\right|^{2}=\left|u_{*}\left(\psi_{*}\left(\bar{\partial}_{i}\right)\right)\right|^{2} \\
& =\pi\left(\psi_{, i}^{l} \partial_{l}, \psi_{, i}^{m} \partial_{m}\right) \\
& =\pi_{l m} \psi_{, i}^{l} \psi_{, i}^{m} .
\end{aligned}
$$

(Here we have used $\left\{\bar{\partial}_{i}\right\}$ for the basis vectors in $\Omega_{1}$.) Thus $(2.3 \mathrm{v})$ holds when $i=j$ and the general result follows from the definition (2.3ii) applied to $\pi_{v}\left(\bar{\partial}_{i}, \bar{\partial}_{j}\right)$ and from linearity.

It remains to verify (2.3vi). We note that the function $|\nabla u|^{2}$ and the function $g^{i j} \pi_{i j}$ are both coordinate-invariant, the first by definition, and the second because of the tensorial-transformation law (2.3v) for $\pi$ and $g$. Now, if $g$ is the Euclidean metric $\delta_{i j}$, then we have from Theorem 1.10 and the normalization $(1.10 \mathrm{v})$ that

$$
|\nabla u|^{2}=\frac{1}{\omega_{n}} \int_{S^{n-1}} e_{\omega}(x) d \sigma(\omega) .
$$

Writing $\omega=\omega^{i} \partial_{i}$ and applying (2.3iv) we see that

$$
|\nabla u|^{2}=\frac{1}{\omega_{n}} \int_{S^{n-1}} \pi_{i j} \omega^{i} \omega^{j} d \sigma(\omega) .
$$

Since

$$
\frac{1}{\omega_{n}} \int_{S^{n-1}} \omega^{i} \omega^{j} d \sigma(\omega)=\delta^{i j}
$$

we see that (2.3vi) holds in the case of a Euclidean metric. The general result follows because in small neighborhoods one can always pick almost-Euclidean local coordinates. By continuity of $|\nabla u|^{2}$ with respect the the metric (Theorem 1.11), we see that in such a local-coordinate chart, (2.3vi) almost holds. Thus the functions $|\nabla u|^{2}$ and $g^{i j} \pi_{i j}$ are arbitrarily close, so they are equal.

2.4. Geodesic homotopies and interior Lipschitz continuity. In this section we deduce the interior Lipschitz continuity of Dirichlet Problem solutions, as a consequence of a weak version of the Bochner inequality for $\Delta|\nabla u|^{2}$, 
which states that $|\nabla u|^{2}$ is almost subharmonic. Using refinements of the arguments given here we will derive much more precise estimates in the sequel, not only for $\Delta|\nabla u|^{2}$, but for $\Delta \pi(V, V)$, where $V$ is any Lipschitz vector field.

In order to motivate the general theorem we recall how one can use finitedifference ideas in the classical case (of harmonic functions on Euclidean domains) in order to deduce interior gradient bounds. The proof in the general case will follow the same idea but will be complicated both by the fact that the target is more general, and by the fact that the domain is non-Euclidean. Because we do not want to assume any linear structure on the target we will phrase our finite-difference argument in terms of energy inequalities between our solution and certain compactly-supported distortions of it which also make sense in the NPC setting.

Let $\Omega$ be a Euclidean domain, and let $u$ be the solution to the Dirichlet problem, as in section 2.2. Let $\eta \in C_{0}^{\infty}(\Omega), \eta \geq 0$. Let $w$ be a constant vector of small magnitude, and define

$$
u_{w}(x)=u(x+w) .
$$

As long as $|w|<\operatorname{dist}(\operatorname{supp}(\eta), \partial \Omega)$, the function $(1-\eta) u+\eta u_{w}$ is an admissable comparison function, and we have

$$
\int_{\Omega}|\nabla u|^{2} d \mu \leq \int_{\Omega}\left|\nabla\left((1-\eta) u+\eta u_{w}\right)\right|^{2} d \mu .
$$

But since $u_{w}$ is also harmonic we have a symmetric inequality

$$
\int_{\Omega_{w}}\left|\nabla u_{w}\right|^{2} d \mu \leq \int_{\Omega_{w}}\left|\nabla\left((1-\eta) u_{w}+\eta u\right)\right|^{2} .
$$

Adding these two inequalities, cancelling the zero ${ }^{\text {th }}$ order terms, and collecting terms which are first and second order in $\eta$, we get:

$$
\begin{aligned}
0 \leq & \int-\nabla \eta \bullet \nabla\left(u-u_{w}\right)^{2}-2 \int \eta\left|\nabla\left(u-u_{w}\right)\right|^{2} \\
& +2 \int|\nabla \eta|^{2}\left(u-u_{w}\right)^{2}+2 \int \eta^{2}\left|\nabla\left(u-u_{w}\right)\right|^{2}+\int \nabla\left(\eta^{2}\right) \bullet \nabla\left(u-u_{w}\right)^{2} .
\end{aligned}
$$


Noting that the quadratic terms are all well-defined, we deduce (by considering variations by $t \eta$ as $t \rightarrow 0^{+}$)

$$
0 \leq \int-\nabla \eta \bullet \nabla\left(u-u_{w}\right)^{2}-2 \int \eta\left|\nabla\left(u-u_{w}\right)\right|^{2} .
$$

But this is just the statment that

$$
\Delta\left(u_{w}-u\right)^{2}-2\left|\nabla\left(u_{w}-u\right)\right|^{2} \geq 0
$$

weakly. (Note, that in the present case we actually deduce the equality in $(2.4 \mathrm{vi})$, since we are able to consider two-sided variations ( $t$ positive and negative). This will not be possible for a general NPC target.) In particular, $\left(u-u_{w}\right)^{2}$ is subharmonic, so its value at the center of a ball of radius $R$ is bounded by its average value on the entire ball. By monotonicity of the approximate-energy functionals (or by directional derivative theory (1.9), this average value is bounded above by a dimensional constant time $|w|^{2} E / R^{n}$ (where $\mathrm{E}$ is the total energy of the map $u$ ). We deduce that $u$ is Lipschitz continuous, with constant depending on the distance to the boundary of $\Omega$.

We remark that by considering displacements $t w$ with $t \rightarrow 0^{+}$, we are able to deduce from $(2.4 \mathrm{vi})$ that

$$
\Delta\left|u_{*}(w)\right|^{2} \geq 0
$$

weakly. By then averaging over all directions in the unit sphere (or ball), we conclude that

$$
\Delta|\nabla u|^{2} \geq 0
$$

weakly. One could use this last inequality to deduce the Lipschitz estimate, and that is the strategy we will follow in case our domain is non-Euclidean. In that case our argument will be complicated by the fact that it is not possible to pick Killing translation fields (like $w$ above) and so there will be error terms in our estimate.

In order apply the above arguments to maps into NPC spaces, we must first prove some lemmas showing that the analogs of the maps $(1-\eta) u+\eta u_{w}$ behave well in the general setting. For $u_{0}, u_{1} \in W^{1,2}(\Omega, X), 0 \leq t \leq 1$ and $x \in \Omega$ define $u_{t}(x)$ to be the point which is the fraction $t$ of the way along the geodesic from $u_{0}(x)$ to $u_{1}(x)$. 
Lemma 2.4.1. Let $(\Omega, g)$ be a Lipschitz Riemannian domain and let $(X, d)$ be an NPC metric space. Let $u_{0}, u_{1} \in W^{1,2}(\Omega, X)$ and let $\eta \in C^{0,1}(\bar{\Omega})$ be a Lipschitz function, $0 \leq \eta \leq 1$. Define

$$
u_{\eta}(x) \equiv\left((1-\eta) u_{0}+\eta u_{1}\right)(x) \equiv u_{\eta(x)}(x) .
$$

Then $u_{\eta} \in W^{1,2}(\Omega, X)$.

Proof. This lemma follows immediately from the convexity of distances under geodesic homotopy and from the triangle inequality. Indeed, we have the estimate

$$
\begin{aligned}
& d^{2}\left(u_{\eta(y)}(y), u_{\eta(x)}(x)\right) \\
\leq & 4 d^{2}\left(u_{\eta(x)}(x), u_{\eta(x)}(y)\right)+4 d^{2}\left(u_{\eta(x)}(y), u_{\eta(y)}(y)\right) \\
\leq & 4\left[d^{2}\left(u_{0}(x), u_{0}(y)\right)+d^{2}\left(u_{1}(x), u_{1}(y)\right)+(\eta(y)-\eta(x))^{2} d^{2}\left(u_{0}(y), u_{1}(y)\right)\right]
\end{aligned}
$$

which implies that $u_{\eta}$ is a finite-energy map.

Lemma 2.4.2. Let $u_{0}, u_{1}, \eta$ be as above, with $0 \leq \eta<\frac{1}{2}$. Use subscripts on $\pi$ to indicate which map is being used to compute the particular tensor. Then, considered as bilinear forms we have the following inequality between the various $\pi$ 's:

$$
\pi_{u_{\eta}}+\pi_{u_{1-\eta}} \leq \pi_{u_{0}}+\pi_{u_{1}}-\nabla \eta \otimes d^{2}\left(u_{0}, u_{1}\right)+Q(\eta, \nabla \eta) .
$$

Here $Q(\eta, \nabla \eta)$ consists of integrable terms which are quadratic in $\eta$ and $\nabla \eta$.

Proof. Define

$$
\eta_{-}=\min (\eta(x), \eta(y)) \quad \eta^{+}=\max (\eta(x), \eta(y)) .
$$

If $\eta_{-}=\eta(y)$ we consider the ordered sequence

$$
\left\{u_{\eta_{-}}(y), u_{\eta_{-}}(x), u_{1-\eta_{-}}(x), u_{1-\eta_{-}}(y)\right\}
$$

take

$$
t=\frac{\eta(x)-\eta(y)}{1-2 \eta(y)}
$$


and apply $(2.1 \mathrm{v})$. In case $\eta_{-}=\eta(x)$ we interchange the roles of $x$ and $y$ and also apply $(2.1 \mathrm{v})$. In both cases we deduce

$$
\begin{aligned}
& d^{2}\left(u_{\eta}(y), u_{\eta}(x)\right)+d^{2}\left(u_{1-\eta}(y), u_{1-\eta}(x)\right) \\
& \leq d^{2}\left(u_{\eta_{-}}(y), u_{\eta_{-}}(x)\right)+d^{2}\left(u_{1-\eta_{-}}(y), u_{1-\eta_{-}}(x)\right) \\
& \quad-(\eta(y)-\eta(x))\left(d^{2}\left(u_{0}(y), u_{1}(y)\right)-d^{2}\left(u_{0}(x), u_{1}(x)\right)\right)(1-2 \eta(y)) \\
& \quad+2\left(d^{2}\left(u_{0}(x), u_{1}(x)\right)+d^{2}\left(u_{0}(y), u_{1}(y)\right)\right)\left(\frac{\eta(y)-\eta(x)}{1-2 \eta(y)}\right)^{2} .
\end{aligned}
$$

It follows from the geodesic convexity statement (2.1iv) that

(2.4viii) $\quad d^{2}\left(u_{\eta_{-}}(y), u_{\eta_{-}}(x)\right)+d^{2}\left(u_{1-\eta_{-}}(y), u_{1-\eta_{-}}(x)\right)$

$$
\leq d^{2}\left(u_{0}(y), u_{0}(x)\right)+d^{2}\left(u_{1}(y), u_{1}(x)\right)
$$

Let $Z \in \Gamma(T \bar{\Omega})$ be a Lipschitz vector field. By taking $y=\bar{x}(x, \varepsilon)$, integrating (2.4vii) against a non-negative $f \in C_{c}(\Omega)$, noting (2.4viii), averaging with respect to $\varepsilon$, and letting $\varepsilon \rightarrow 0$, we deduce

$$
\begin{aligned}
& \left|\left(u_{\eta}\right)_{*}(Z)\right|^{2}+\left|\left(u_{1-\eta}\right)_{*}(Z)\right|^{2} \\
& \quad \leq\left|\left(u_{0}\right)_{*}(Z)\right|^{2}+\left|\left(u_{1}\right)_{*}(Z)\right|^{2}-\eta_{*}(Z)\left(d^{2}\left(u_{0}, u_{1}\right)\right)_{*}(Z)+Q(\eta, \nabla \eta),
\end{aligned}
$$

which is the claim of our Lemma. In this deduction we have used the fact that $d^{2}\left(u_{0}, u_{1}\right)$ is a real-valued Sobolev function (Corollary 1.6.3), and that if $\eta$ and $h$ are two functions in $W^{1,2}(\Omega, \mathbb{R})$, then the measure $\eta_{*}(Z) h_{*}(Z) d \mu$ is the weak limit of the expressions

$$
\frac{(\eta(y)-\eta(x))(h(y)-h(x))}{\varepsilon^{2}} d \mu
$$

(where $y=\bar{x}(x, \varepsilon)$ ) as $\varepsilon \rightarrow 0$. This last fact follows immediately from the identities

$$
\left|(\eta+h)_{*}(Z)\right|^{2}=\left|\eta_{*}(Z)\right|^{2}+\left|h_{*}(Z)\right|^{2}+2 \eta_{*}(Z) h_{*}(Z)
$$

and

$$
\begin{aligned}
& ((\eta+h)(y)-(\eta+h)(x))^{2} \\
& \quad=(\eta(y)-\eta(x))^{2}+(h(y)-h(x))^{2}+2(\eta(y)-\eta(x))(h(y)-h(x)) .
\end{aligned}
$$

Thus Lemma 2.4.2 is complete. 
Remark 2.4.3. In case $\Omega$ is a Euclidean domain we can use Lemma 2.4.2 to conclude that a harmonic map is Lipschitz continuous in the interior, in direct analogy with the argument given at the beginning of this section. Indeed, we take $w$ to be a constant vector and define $u_{w}(x)$ as in (2.4i). Then Lemma 2.4.1 and the minimizing property of harmonic maps imply that (2.4ii) and (2.4iii) both hold. We sum these two inequalities and use Lemma 2.4.2 to expand the right-hand side. (Take the trace of the bilinear-form inequality, i.e. apply it to the basis vectors $\partial_{i}$ and sum over $i$.) This yields

$$
\begin{aligned}
\int_{\Omega}|\nabla u|^{2}+\int_{\Omega_{w}}\left|\nabla u_{w}\right|^{2} \leq \int_{\Omega}\left|\nabla u_{\eta}\right|^{2}+\int_{\Omega_{w}}\left|\nabla u_{1-\eta}\right|^{2} \\
\quad \leq \int_{\Omega}|\nabla u|^{2}+\int_{\Omega_{w}}\left|\nabla u_{w}\right|^{2}-\int_{\Omega} \nabla d^{2}\left(u, u_{w}\right) \bullet \nabla \eta+\int_{\Omega} Q(\eta, \nabla \eta) .
\end{aligned}
$$

Cancelling the zero ${ }^{\text {th }}$-order terms, replacing $\eta$ with $t \eta$, and letting $t \rightarrow 0$ as before, we deduce the weak subharmonicity statement which compares to (2.4vi):

$$
\Delta d^{2}\left(u, u_{w}\right) \geq 0 .
$$

This implies the interior Lipschitz continuity. Note, what we are really showing (and using) here is the fact that whenever $u_{0}$ and $u_{1}$ are harmonic with respect to the same domain metric, then $d^{2}\left(u_{0}, u_{1}\right)$ is subharmonic.

Unfortunately, for a non-Euclidean domain we must estimate somewhat more carefully. In particular, it is convenient to introduce another integrable tensor. In the case of real-valued maps $u_{0}, u_{1}$, it corresponds to $\mid(1-$ $\eta)\left(u_{0}\right)_{*}(Z)+\left.\eta\left(u_{1}\right)_{*}(Z)\right|^{2}$.

Lemma 2.4.4. Given $u_{0}, u_{1} \in W^{1,2}(\Omega, X)$ and $\eta \in C(\Omega)$, with $0 \leq \eta \leq 1$, there exists a symmetric bilinear integrable tensor $\mathcal{P}\left(u_{0}, u_{1}, \eta\right)$ defined on the subset $\{0<\eta<1\} \subset \Omega$, as follows: For any $Z \in \Gamma(T \bar{\Omega})$,

$$
\frac{d^{2}\left(u_{\eta(x)}(x), u_{\eta(x)}(\bar{x}(x, \varepsilon))\right)}{\varepsilon^{2}} d \mu \rightarrow \mathcal{P}\left(u_{0}, u_{1}, \eta\right)(Z, Z) d \mu
$$

on the subset $0<\eta<1$.

Proof. The reason that $\mathcal{P}$ exists is the convexity property of $d^{2}\left(P_{t}, Q_{t}\right)(2.1 \mathrm{iv})$ on geodesic paths. Since $d^{2}$ is always non-negative one immediately deduces 
that it is Lipschitz continuous away from $t=0,1$. In fact, for $0<s<t<1$ we have the estimate

$$
\frac{s-t}{1-s} d^{2}\left(P_{0}, Q_{0}\right) \leq d^{2}\left(P_{t}, Q_{t}\right)-d^{2}\left(P_{s}, Q_{s}\right) \leq \frac{t-s}{1-t} d^{2}\left(P_{1}, Q_{1}\right) .
$$

Now, let $f \geq 0$ be continuous and have support in $\{\delta<\eta(x)<1-\delta\}$, for some $\delta>0$. Assume first that $\eta \in C_{0}^{\infty}(\Omega)$. Given any $\Delta t>0$ we may partition a subset of the interval $(\delta, 1-\delta)$ containing the support of $f$, into a collection of intervals $\left\{\left(t_{i-1}, t_{i}\right]\right\}$ so that the $t_{i}$ are regular values of $\eta$, and so that the norm of the partition (the length of the longest interval) is at most $\Delta t$.

For $Z \in \Gamma(T \bar{\Omega})$ and for $\varepsilon$ and $x$ given, we write $y$ for $\bar{x}(x, \varepsilon)$. Using this convention we make the following definitions:

$$
\begin{aligned}
\mathcal{L}_{\varepsilon}(f) & =\int_{\Omega} f(x) \frac{d^{2}\left(u_{\eta(x)}(x), u_{\eta(x)}(y)\right)}{\varepsilon^{2}} d \mu \\
\Omega_{i} & =\left\{x \mid t_{i-1}<\eta(x) \leq t_{i}\right\} \\
\mathcal{L}_{\varepsilon}^{i}(f) & =\int_{\Omega_{i}} f(x) \frac{d^{2}\left(u_{t_{i}}(x), u_{t_{i}}(y)\right)}{\varepsilon^{2}} d \mu \\
\mathcal{L}^{i}(f) & =\int_{\Omega_{i}} f(x)\left|\left(u_{t_{i}}\right)_{*}(Z)\right|^{2} d \mu \\
\mathcal{M}_{\varepsilon}(f) & =\int_{\Omega} f(x) \frac{d^{2}\left(u_{0}(x), u_{0}(y)\right)+d^{2}\left(u_{1}(x), u_{1}(y)\right)}{\varepsilon^{2}} d \mu \\
\mathcal{M}(f) & =\int_{\Omega} f(x)\left(\left|\left(u_{0}\right)_{*}(Z)\right|^{2}+\left|\left(u_{1}\right)_{*}(Z)\right|^{2}\right) d \mu .
\end{aligned}
$$

It is clear that

$$
\lim _{\varepsilon \rightarrow 0} \mathcal{M}_{\varepsilon}(f)=\mathcal{M}(f) .
$$

Because the $\Omega_{i}$ have smooth boundaries it is easy to show that

$$
\lim _{\varepsilon \rightarrow 0} \mathcal{L}_{\varepsilon}^{i}(f)=\mathcal{L}^{i}(f)
$$

From the continuity estimate $(2.4 \mathrm{x})$ we deduce

$$
\begin{aligned}
\sum_{i} \mathcal{L}_{\varepsilon}^{i}(f)-\frac{\Delta t}{\delta} \mathcal{M}_{\varepsilon}(f) & \leq \mathcal{L}_{\varepsilon}(f) \\
& \leq \sum_{i} \mathcal{L}_{\varepsilon}^{i}(f)+\frac{\Delta t}{\delta} \mathcal{M}_{\varepsilon}(f)
\end{aligned}
$$


Letting $\varepsilon \rightarrow 0$ in $(2.4 \mathrm{xi})$ yields

$$
\begin{aligned}
\sum_{i} \mathcal{L}^{i}(f)-\frac{\Delta t}{\delta} \mathcal{M}(f) & \leq \liminf _{\varepsilon \rightarrow 0} \mathcal{L}_{\varepsilon}(f) \\
& \leq \limsup _{\varepsilon \rightarrow 0} \mathcal{L}_{\varepsilon}(f) \leq \sum_{i} \mathcal{L}^{i}(f)+\frac{\Delta t}{\delta} \mathcal{M}(f)
\end{aligned}
$$

Since $\Delta t$ may be chosen arbitrarily small (2.4xii) implies the existence of a weak limit to the functionals $\mathcal{L}_{\varepsilon}$. The corresponding measure is absolutely continuous with respect to Lebesgue measure (from (2.1iv)), so has an $L^{1}$ density function $\mathcal{P}\left(u_{0}, u_{1}, \eta\right)(Z, Z)$. The bilinearity, symmetry and tensorial properties of $\mathcal{P}$ also follow from (2.4xii), since the functions $\left|\left(u_{t_{i}}\right)_{*}(Z)\right|^{2}$ arise from tensors $\pi_{t_{i}}$ possessing these properties (Lemma 2.4.1).

If $\eta$ is only in $C(\Omega)$, then let $f, \delta$ be as above, and let $\bar{\eta} \in C(\Omega)$, with $|\eta-\bar{\eta}|_{\infty}<\delta / 2$. The inequalities $(2.4 \mathrm{x})$ and (1.4vii) imply

$(2.4$ xiii $) \quad\left|\mathcal{L}_{\varepsilon}^{\eta}(f)-\mathcal{L}_{\varepsilon}^{\bar{\eta}}(f)\right| \leq \frac{2|\eta-\bar{\eta}|_{\infty}}{\delta} \mathcal{M}_{\varepsilon}(f) \leq \frac{2|\eta-\bar{\eta}|_{\infty}}{\delta} \mathcal{M}\left(f_{\varepsilon}^{C}\right)$.

With this estimate one can approximate continuous $\eta$ with smooth $\bar{\eta}$ to deduce the general claim of Lemma 2.4.4.

From the functions $u_{0}, u_{1}, \eta$ as above, and the resulting tensor $\mathcal{P}$ discussed in Lemma 2.4.2, we define another auxiliary tensor $\mathcal{C} \geq 0$ by

(2.4xiv) $\quad \mathcal{C}\left(u_{0}, u_{1}, \eta\right)=\pi_{u_{0}}+\pi_{u_{1}}-\mathcal{P}\left(u_{0}, u_{1}, \eta\right)-\mathcal{P}\left(u_{0}, u_{1}, 1-\eta\right)$.

The following lemma contains the estimates which will enable us to extend our proof of Lipschitz continuity to the general setting.

Lemma 2.4.5. Let $u_{0}, u_{1} \in W^{1,2}(\Omega, X), \eta \in C_{0}^{1}(\Omega)$ with $0 \leq \eta<\frac{1}{2}$. Using $Q(\eta, \nabla \eta)$ to represent integrable quadratic error terms as in our previous Lemma 2.4.2, we have the following estimates on $\{\eta>0\}$ :

$$
\pi_{u_{\eta}}+\pi_{u_{1-\eta}} \leq \pi_{u_{0}}+\pi_{u_{1}}-\mathcal{C}\left(u_{0}, u_{1}, \eta\right)
$$

$$
-\nabla \eta \otimes \nabla d^{2}\left(u_{0}, u_{1}\right)+Q(\eta, \nabla \eta)
$$

$(2.4 \mathrm{xvi}) \quad 0 \leq \eta \pi_{u_{0}}+(1-\eta) \pi_{u_{1}}-\mathcal{P}\left(u_{0}, u_{1}, 1-\eta\right) \leq \mathcal{C}\left(u_{0}, u_{1}, \eta\right)$

(2.4xvii) $\left|\pi_{u_{1-\eta}}-\mathcal{P}\left(u_{0}, u_{1}, 1-\eta\right)\right|$

$$
\leq C|\nabla \eta| d\left(u_{0}, u_{1}\right)\left(\left|\nabla u_{0}\right|_{1}+\left|\nabla u_{1}\right|_{1}\right)+Q(\eta, \nabla \eta) .
$$


Proof. The inequality (2.4xv) follows from Lemma 2.4.4 (also using (2.4xiii)) and the inequality (2.4vii). Notice that $(2.4 \mathrm{xv})$ is an improvement on Lemma 2.4.2. The second inequality (2.4xvi) follows from properties of convex functions. To see why, let $c(t)$ be a convex function defined on the interval $[0,1]$, and define

$$
\mathcal{C}(t)=c(0)+c(1)-c(t)-c(1-t) .
$$

Then the inequalities

$$
0 \leq t c(0)+(1-t) c(1)-c(1-t) \leq \mathcal{C}(t)
$$

hold, since the first one follows immediately from convexity and the second one reduces to the other convexity statement

$$
c(t) \leq(1-t) c(0)+t c(1) .
$$

Taking $t=\eta(x), y=\bar{x}(x, \varepsilon) c(t)=d^{p}\left(u_{t}(x), u_{t}(y)\right.$, integrating and averaging against suitable $f$, and applying (2.4xviii) gives the conclusion (2.4xvi).

We are left to show the final estimate (2.4xvii). From the triangle inequality we have

$$
\begin{aligned}
(2.4 \mathrm{xix}) \quad d\left(u_{1-\eta(x)}(x), u_{1-\eta(x)}(y)-|\eta(y)-\eta(x)| d\left(u_{0}(y), u_{1}(y)\right)\right. \\
\leq d\left(u_{1-\eta(x)}(x), u_{1-\eta(y)}(y)\right) \\
\leq d\left(u_{1-\eta(x)}(x), u_{1-\eta(x)}(y)\right)+|\eta(y)-\eta(x)| d\left(u_{0}(y), u_{1}(y)\right) .
\end{aligned}
$$

The inequality (2.4xvii) follows from (2.4xix) upon squaring, integrating and averaging, and letting $\varepsilon \rightarrow 0$. Letting the reader check the details, we note that the argument uses the estimates

$$
\begin{aligned}
d\left(u_{t}(x), u_{t}(y)\right) & \leq d\left(u_{0}(x), u_{0}(y)\right)+d\left(u_{1}(x), u_{1}(y)\right), \\
|\eta(y)-\eta(x)| & \leq \varepsilon|Z|_{\infty}\left(|\nabla \eta(x)|+o_{\varepsilon}(1)\right),
\end{aligned}
$$

and the fact that the $p=1$ approximate energy functions of a $p=2$ Sobolev mapping, when integrated against an $L^{2}$ function $h$, converge to the integral of the limit $p=1$ energy density times $h$. This completes the estimates of Lemma 2.4.5. 
Theorem 2.4.6. Let $(\Omega, g)$ be a Lipschitz Riemannian domain, and let $u$ solve the Dirichlet problem, as in Theorem 2.2. Then $u$ is a locally Lipschitz continuous function in the interior of $\Omega$, where the local Lipschitz constant is bounded above by

$$
C\left(\frac{E}{\min \left(1, \operatorname{dist}(x, \partial \Omega)^{n}\right)}\right)^{\frac{1}{2}},
$$

where $C$ is a constant which depends only on dimension $n$ and on the regularity of the metric $g$, and where $E$ is the total energy of the map $u$.

Proof. Let $w$ be a unit vector field defined on a local coordinate chart of $\Omega$. Write $u_{s w}(x)=u(\bar{x}(x, s))$. The function $u_{s w}$ is harmonic with respect to the pull-back metric $g_{s w}=\bar{x}^{*}(g)$. Let $\eta \in C_{0}^{2}(\Omega)$, with $0 \leq \eta<\frac{1}{2}$. Assume (for technical reasons) that $\partial\{\eta>0\}$ is a set of zero Lebesgue measure. Take $u_{0}=u, u_{1}=u_{s w}$ and define $u_{\eta}$ as above. Assuming that $s$ is sufficiently small we may write the analog of the sum of (2.4ii) and (2.4iii) as

$$
\int_{\Omega}\left(\pi_{u_{0}}\right)_{i j} g^{i j} d \mu+\int_{\Omega}\left(\pi_{u_{1}}\right)_{i j}\left(g^{i j} d \mu\right)_{s w} \leq \int_{\Omega}\left(\pi_{u_{\eta}}\right)_{i j} g^{i j} d \mu+\int_{\Omega}\left(\pi_{u_{1-\eta}}\right)_{i j}\left(g^{i j} d \mu\right)_{s w} .
$$

We may regroup the terms as follows:

$$
\begin{aligned}
\int_{\Omega}\left(\pi_{u_{0}}+\pi_{u_{1}}\right)_{i j} g^{i j} d \mu \leq \int_{\Omega}\left(\pi_{u_{\eta}}+\pi_{u_{1-\eta}}\right)_{i j} g^{i j} d \mu \\
\quad+\int_{\Omega}\left(\pi_{u_{1-\eta}}-\pi_{u_{1}}\right)_{i j}\left(\left(g^{i j} d \mu\right)_{s w}-g^{i j} d \mu\right)
\end{aligned}
$$

If we define the functions $\mathcal{P}$ and $\mathcal{C}$ to be zero on $\{\eta=0\}$, then because of our assumption that $\partial\{\eta>0\}$ has measure zero, we may deduce from Lemma 2.4.5 that

$$
\begin{aligned}
\int_{\Omega}\left(\pi_{u_{\eta}}+\right. & \left.\pi_{u_{1-\eta}}\right)_{i j} g^{i j} d \mu \\
\leq \int_{\Omega}\left(\pi_{u_{0}}+\pi_{u_{1}}\right)_{i j} g^{i j} d \mu & -\int_{\Omega} \nabla \eta \bullet \nabla d^{2}\left(u, u_{s w}\right) d \mu \\
& -\int_{\Omega} \mathcal{C}\left(u_{0}, u_{1}, \eta\right)_{i j} g^{i j} d \mu+Q(\eta, \nabla \eta) .
\end{aligned}
$$


To estimate the other terms in $(2.4 \mathrm{xx})$ we write

$$
\begin{aligned}
\pi_{u_{1-\eta}}-\pi_{u}= & \pi_{u_{1-\eta}}-\mathcal{P}\left(u_{0}, u_{1}, 1-\eta\right) \\
& +\mathcal{P}\left(u_{0}, u_{1}, 1-\eta\right)-\eta \pi_{u_{0}}-(1-\eta) \pi_{u_{1}}+\eta\left(\pi_{u_{1}}-\pi_{u_{0}}\right) .
\end{aligned}
$$

The last term of (2.4xxii) contributes a term in (2.4xx) which may be rewritten by changing variables in part of the expression, from $x$ to $\bar{x}(x, s)$ :

$$
\begin{aligned}
\int_{\Omega} \eta\left(\pi_{u_{s w}}-\pi_{u}\right)_{i j}( & \left.\left(g^{i j} d \mu\right)_{s w}-g^{i j} d \mu\right) \\
= & \int_{\Omega} \eta\left(\pi_{u}\right)_{i j}\left(2 g^{i j} d \mu-\left(g^{i j} d \mu\right)_{s w}-\left(g^{i j} d \mu\right)_{-s w}\right) \\
& +\int_{\Omega}\left(\pi_{u}\right)_{i j}\left(\eta_{-s w}-\eta\right)\left(g^{i j} d \mu-\left(g^{i j} d \mu\right)_{-s w}\right)
\end{aligned}
$$

Combining (2.4xx)-(2.4xxiii) with the estimates of Lemma 2.4.5 we see

(2.4xxiv)

$$
\begin{aligned}
0 \leq & \int_{\Omega} d^{2}\left(u, u_{s w}\right) \Delta \eta d \mu-\int_{\Omega} \mathcal{C}\left(u_{0}, u_{s w}, \eta\right)_{i j} g^{i j} d \mu \\
& +C s \int_{\Omega}|\nabla \eta| d\left(u, u_{s w}\right)\left(|\nabla u|_{1}+\left|\nabla u_{s w}\right|_{1}\right) d \mu \\
& +C s \int_{\Omega}\left|\mathcal{C}\left(u_{0}, u_{s w}, \eta\right)\right| d \mu+C s^{2} \int_{\Omega}(\eta+|\nabla \eta|)|\nabla u|^{2} d \mu .
\end{aligned}
$$

Dividing the inequality (2.4xxiv) by $s^{2}$, averaging over a frame of unit vectors $w$, and letting $s \rightarrow 0$ we deduce

$$
\int_{\Omega}|\nabla u|^{2}(\Delta \eta+C|\nabla \eta|+C \eta) d \mu \geq 0 .
$$

(Note that we may remove the technical condition that $\partial\{\eta>0\}$ have zero Lebesgue measure by an approximation argument, once $(2.4 \mathrm{xxv})$ is established for functions satisfying this requirement.) This last inequality says that $|\nabla u|^{2}$ is essentially subharmonic. It is well known (see e.g. [Mo]) that this kind of differential inequality implies that the essential supremum of $|\nabla u|^{2}$ in a ball of radius $\frac{R}{2}$ is bounded by a constant times its average value on the concentric radius $R$ ball, as long as $R \leq 1$. (One can also show this by using radiallysymmetric test functions in $(2.4 \mathrm{xxv})$ to deduce a differential inequality for the 
integral averages of $|\nabla u|^{2}$ over radius $r$ spherical shells centered at points in the $\frac{R}{2}$ ball.) Hence the directional derivative energies $\left|u_{*}(Z)\right|^{2}$ are similarly bounded (for bounded vector fields $Z$.) Working with the local-coordinate field $\partial_{1}$ we construct a representative of $u$ which is locally Lipschitz continuous in the $\partial_{1}$ direction, using the techniques and results of Lemma 1.9.1. Working inductively in successive directions $\partial_{2}, \ldots, \partial_{n}$, we construct a representative of $u$ which is Lipschitz continuous in the $\frac{R}{2}$ ball, with Lipschitz constant bounded by a multiple of the bound for $|\nabla u|$. For a given $x \in \Omega$ we may choose $R$ to be the minimum of $d(x, \partial \Omega)$ and 1 . The resulting estimate for the Lipschitz constant is exactly the claim of our Theorem.

Remark 2.4.7. Wherever the boundary of $\Omega$ is smooth and the boundary data are $C^{\alpha}(0<\alpha<1)$, T. Serbinowski has shown that the solution $u$ extends to be $C^{\alpha}$ up to the boundary, with the $C^{\alpha}$ norm depending on the boundary and on the total energy of the map [Se].

2.5. Center of mass constructions. We recall that averaging works well for maps to NPC spaces $(X, d)$, and that distances between centers of mass can be estimated from above in terms of the average distances between the maps. These distance estimates follow from the quadrilateral comparison lemmas of [Re] which we discussed in $\S 2.1$. In the next section we will convert the distance estimates into Lipschitz and energy bounds for various auxiliary Sobolev maps related to the study of equivariant harmonic maps.

Note that the definition of $L^{2}$ maps given in (1.1) really only requires the domain to be a measure space, as long as one restricts to separable maps for which inverse images of open sets are measurable. In this generality we consider a domain measure space $\mathcal{M}$, and a probability measure $\nu$ defined on $\mathcal{M}$ (i.e. $\nu$ is non-negative with total mass 1 ).

Lemma 2.5.1. Let $(\mathcal{M}, \nu)$ be a probability measure space, let $(X, d)$ be an $N P C$ space, and let $f \in L^{2}(\mathcal{M}, X)$. Then there exists a unique center of mass $\bar{f}=\bar{f}_{\nu}$ for $f$, defined as the point in $X$ which minimizes the integral

$$
I_{f, \nu}(Q)=\int_{\mathcal{M}} d^{2}(f(m), Q) d \nu(m) .
$$


Proof. We claim that the integral above is uniformly convex in $Q$, so that any minimizing sequence converges to a (unique) limit. Indeed, if $P_{0}, P_{1}$ are two points in $X$, with midpoint $P_{\frac{1}{2}}$, then triangle comparison (2.1ii) yields

$$
d^{2}\left(f(m), P_{\frac{1}{2}}\right) \leq \frac{1}{2} d^{2}\left(f(m), P_{0}\right)+\frac{1}{2} d^{2}\left(f(m), P_{1}\right)-\frac{1}{4} d^{2}\left(P_{0}, P_{1}\right) .
$$

Integrating over $\mathcal{M}$ we obtain

$$
\frac{1}{4} d^{2}\left(P_{0}, P_{1}\right) \leq \frac{1}{2}\left[I\left(P_{0}\right)+I\left(P_{1}\right)\right]-I\left(P_{\frac{1}{2}}\right) .
$$

Thus any minimizing sequence $\left\{P_{i}\right\}$ is Cauchy, so the integral attains its minimum for a unique point in $X$.

Proposition 2.5.2. Let $\mathcal{M}$ be a measure space, and let $\nu, \nu^{\prime}$ be two probability measures on $\mathcal{M}$. Suppose $f, h$ are in $L^{2}(\mathcal{M}, X)$ for both measure choices. Write $\bar{f}$ for $\bar{f}_{\nu}$ and $\bar{h}$ for $\bar{h}_{\nu^{\prime}}$. Then for any $0 \leq \alpha \leq 1$ we have the estimate

$$
\begin{aligned}
d^{2}(\bar{f}, \bar{h}) \leq & \int_{\mathcal{M}} d^{2}(f, h) d \nu-\alpha \int_{\mathcal{M}}[d(f, h)-d(\bar{f}, \bar{h})]^{2} d \nu-(1-\alpha) \int_{\mathcal{M}}[d(f, \bar{f}) \\
& -d(h, \bar{h})]^{2} d \nu+2 d(\bar{f}, \bar{h}) \int_{\mathcal{M}} d(h, \bar{h})\left|d \nu-d \nu^{\prime}\right| .
\end{aligned}
$$

Proof. Consider the geodesic from $Q=\bar{f}$ to $R=\bar{h}$, and write $Q_{t}$ for the point which is a fraction $t$ of the distance between $Q$ and $R$. Since

$$
I_{f, \nu}(\bar{f}) \leq I_{f, \nu}\left(Q_{t}\right), \quad I_{h, \nu^{\prime}}(\bar{h}) \leq I_{h, \nu^{\prime}}\left(Q_{1-t}\right)
$$

we have

$$
\begin{aligned}
\int d^{2}(f, \bar{f})+d^{2}(h, \bar{h}) d \nu \leq \int & d^{2}\left(f, Q_{t}\right)+d^{2}\left(h, Q_{1-t}\right) d \nu \\
& +\int\left[d^{2}(h, \bar{h})-d^{2}\left(h, Q_{1-t}\right)\right]\left(d \nu-d \nu^{\prime}\right) .
\end{aligned}
$$

Estimate the integrand which appears in the first term of the right side by using Euclidean distance comparison: For each $m \in \mathcal{M}$ we construct the quadrilateral with consecutive vertices $f(m), \bar{f}, \bar{h}, h(m)$ and apply (2.1v):

$$
\begin{aligned}
d^{2}\left(f, Q_{t}\right)+d^{2}\left(h, Q_{1-t}\right) \leq d^{2} & (f, \bar{f})+d^{2}(h, \bar{h}) \\
& +t\left[d^{2}(f, h)-d^{2}(\bar{f}, \bar{h})\right]-t\left(\alpha[d(f, h)-d(\bar{f}, \bar{h})]^{2}\right. \\
& \left.+(1-\alpha)[d(f, \bar{f})-d(h, \bar{h})]^{2}\right)+2 t^{2} d^{2}(\bar{f}, \bar{h}) .
\end{aligned}
$$


Integrating (2.5ii) with respect to $\nu$ yields a bound for the first term on the right of (2.5i). We may bound the second term by writing

$$
d^{2}(h, \bar{h})-d^{2}\left(h, Q_{1-t}\right)
$$

as a difference of squares, using the fact that

$$
d\left(\bar{h}, Q_{1-t}\right)=\operatorname{td}(\bar{f}, \bar{h})
$$

and applying the triangle inequality in the difference term. The resulting upper bound is

$$
t d(\bar{f}, \bar{h}) \int\left[d(h, \bar{h})+d\left(h, Q_{1-t}\right)\right]\left|d \nu-d \nu^{\prime}\right| .
$$

Use these estimates in (2.5i) and note that the terms which are order zero in $t$ cancel. Divide the resulting inequality by $t$. Proposition 2.5 .2 is the limit inequality as $t \rightarrow 0$.

Remark 2.5.3. Note that Proposition 2.5.2 is the natural generalization of the distance convexity statement (2.2iii) which lies at the heart of the solution to Dirichlet's problem. Indeed, (2.2iii) is the case of Proposition 2.5.2 in which the measure space consists of two points $a, b$, each of mass $\frac{1}{2}\left(\nu=\nu^{\prime}\right)$ and $\alpha=0$. The maps $f, h$ are given by

$$
\begin{aligned}
& f(a)=u(x), f(b)=v(x), \bar{f}=w(x) \\
& h(a)=v(y), h(b)=v(y), \bar{h}=w(y) .
\end{aligned}
$$

More generally, if $u \in L^{2}(M \times \mathcal{M}, X)$ is any parameterized family of maps, then one can construct the average map analogously. In this case we take any probability measure $\nu=\nu^{\prime}$ defined on $\mathcal{M}$, take $f(\lambda)=u(x, \lambda)$ and $h(\lambda)=$ $u(y, \lambda)$. Applying Proposition 2.5.2 with $\alpha=0$ we deduce (after integrating over the set $|x-y|=\varepsilon$, averaging, and letting $\varepsilon \rightarrow 0$ ) the generalization of (2.2vi):

$$
E^{\bar{u}} \leq \int_{\mathcal{M}} E^{u_{\lambda}} d \nu(\lambda)-\int_{\mathcal{M}} \int_{M}\left|\nabla d\left(u_{\lambda}(x), \bar{u}(x)\right)\right|^{2} d \mu(x) d \nu(\lambda) .
$$

(We have written $u_{\lambda}(x)$ for $u(x, \lambda)$.)

Finally, we recall the distance decreasing property of projection onto convex subsets of NPC spaces $X$. This fact is well-known, at least in the case of 
Riemannian NPC $X$. In metric space generality it is easiest to prove with quadrilateral comparisons.

Proposition 2.5.4. Let $K$ be a closed, geodesically convex subset of the NPC space $X$. Then there is a well-defined nearest-point projection map $\pi: X \rightarrow K$ so that

$$
d\left(\pi\left(P_{0}\right), \pi\left(P_{1}\right)\right) \leq d\left(P_{0}, P_{1}\right)
$$

for all $P_{0}, P_{1} \in X$. In particular, if $\mathcal{M}, \nu, f$ are as in Lemma 2.5.1, and if the range of $f$ lies in $K$, then so does the center of mass $\bar{f}$.

Proof. The last claim, that the center of mass $\bar{f}$ lies in $K$ whenever of the range of $f$ does, follows from the existence of $\pi$ : Because $\pi$ fixes the range of $f$, its distance decreasing property immediately implies

$$
I_{f, \nu}(\pi(Q)) \leq I_{f, \nu}(Q)
$$

for all $Q \in X$. The uniqueness of $\bar{f}$ then proves this claim.

The existence of the projection map is the fact that every $Q \in X$ has a unique nearest point in $K$ : If $P_{0}, P_{1}$ are in $K$ then so is $P_{\frac{1}{2}}$, and so the reader may verify (as in Lemma 2.5.1) that triangle comparison (2.1ii) forces a (distance to $Q$ ) minimizing sequence $\left\{P_{i}\right\} \subset K$ to be Cauchy.

We now show the distance-decreasing property of $\pi$. Consider the quadrilateral with consecutive verticies $P_{0}, \pi\left(P_{0}\right), \pi\left(P_{1}\right), P_{1}$. Let $Q_{t}$ be the point a fraction $t$ of the way along the geodesic from $\pi\left(P_{0}\right)$ to $\pi\left(P_{1}\right)$. Applying $(2.1 \mathrm{v})$ (as in Proposition 2.5.2) yields

$$
\begin{aligned}
d^{2}\left(P_{0}, Q_{t}\right)+d^{2}\left(P_{1}, Q_{1-t}\right) \leq & d^{2}\left(P_{0}, \pi\left(P_{0}\right)\right)+d^{2}\left(P_{1}, \pi\left(P_{1}\right)\right) \\
& +t\left[d^{2}\left(P_{0}, P_{1}\right)-d^{2}\left(\pi\left(P_{0}\right), \pi\left(P_{1}\right)\right]\right. \\
& +2 t^{2} d^{2}\left(\pi\left(P_{0}\right), \pi\left(P_{1}\right)\right) .
\end{aligned}
$$

Combine this with the competing statement

$$
d^{2}\left(P_{0}, \pi\left(P_{0}\right)\right)+d^{2}\left(P_{1}, \pi\left(P_{1}\right)\right) \leq d^{2}\left(P_{0}, Q_{t}\right)+d^{2}\left(P_{1}, Q_{1-t}\right)
$$

Note that the terms which are order zero in $t$ cancel, divide the resulting inequality by $t$, and let $t \rightarrow 0$ (just as in the proof of Proposition 2.5.2) to deduce Proposition 2.5.4. 
2.6. Equivariant mapping problems. Let $(M, g)$ be a metrically complete Riemannian manifold, possibly with smooth compact boundary $\partial M$. Denote the fundamental group $\pi_{1}(M)$ by $\Gamma$ and the universal cover of $M$ by $\tilde{M}$. Let $X$ be a metric space, and $\rho: \Gamma \rightarrow i \operatorname{som}(X)$ a homomorphism. Such $\rho$ are also called representations of $\Gamma$. We will write $\rho(\gamma) x$ for $\rho(\gamma)(x)$. A particular case of this configuration is the (identity) representation of $\Gamma$ to $i \operatorname{som}(\tilde{M})$, in which $\Gamma$ acts via deck transformations.

A map $u: \tilde{M} \rightarrow X$ is said to be $\Gamma$-equivariant if

$$
u(\gamma x)=\rho(\gamma) u(x)
$$

for all $x \in \tilde{M}$ and $\gamma \in \Gamma$.

For a $\Gamma$-equivariant map $u$ the real-valued functions $d(u(x), u(y))$ are invariant with respect to the domain action. If the map $u$ is locally a Sobolev map, then it follows that the directional and Sobolev energy densities considered in Chapter 1 are $\Gamma$-invariant, so we may think of them as being defined on the quotient $M$.

An equivariant map $u$ is said to be harmonic if it is (locally) a $p=2$ Sobolev map, and if it is stationary for the $p=2$ total energy, defined for locally Sobolev, equivariant $v: \tilde{M} \rightarrow X$ by

$$
E^{v} \equiv \int_{M}|\nabla v|^{2} d \mu
$$

This integral is well-defined as long as $M$ has finite volume. In our case of NPC target $X$ it is clear that the energy convexity statement (2.2vi) holds:

$$
E^{u_{t}} \leq(1-t) E^{u}+t E^{v}-t(1-t) \int_{M}|\nabla d(u, v)|^{2} .
$$

(In fact, the more general statement (2.5iii) holds.) Thus stationary is equivalent to minimizing. (Note, the Dirichlet problem considered in $\S 2.2$ is a special case of equivariant theory, if one takes the homomorphism $\rho$ to be trivial.)

A strategy in studying group representations which has proven useful is to construct harmonic $\Gamma$-equivariant maps, since in many cases the resulting Euler equations (or Bochner formulas) for the maps allow one to deduce information about the representation. (For example, in many cases one can 
prove that the map must be constant and therefore the map's equivariance implies that $\rho(\Gamma)$ has a fixed point.) One consequence of Proposition 2.6.1 below is that in case of compact $M$ the class of finite-energy equivariant maps is non-empty, so the direct method of energy minimization has a chance of producing a harmonic equivariant map.

Assume that $\Gamma$ is finitely generated, say by $\gamma_{1}, \ldots, \gamma_{p}$. (If $M$ is compact this is always the case.) For $P \in X$ define

$$
\delta(P)=\max _{i=1, \ldots, p} d\left(\rho\left(\gamma_{i}\right) P, P\right) .
$$

(It is clear that $\delta$ is a positive function on $X$ if and only if the representation $\rho$ of $\Gamma$ has no fixed points.)

Proposition 2.6.1. Let $M, \Gamma, \rho$ be as above with $\partial M=\emptyset$ and suppose that $X$ is NPC. Then there exists a $\rho$-equivariant locally Lipschitz map $u: \tilde{M} \rightarrow X$. Let $P \in X$ satisfy $\delta(P)=\delta^{\prime}$. If $M$ is compact then $u$ can be constructed to be globally Lipschitz, and there is a constant $C=C(M)$ so that the global bound on the Lipschitz constant $L$ of $u$ is of the form

$$
L \leq C \delta^{\prime}
$$

If $M$ is complete (but not compact) the local Lipschitz constant $L(x)$ of $u(x)$ is bounded by

$$
L(x) \leq C(x) \delta^{\prime}
$$

for a locally bounded function $C(x)$, which depends (only) on the domain manifold $M$.

Proof. Before constructing the map $u$ note that the equality $\delta(P)=\delta^{\prime}$ implies that we may estimate $d(\rho(\gamma) P, P)$ for any $\gamma \in \Gamma$, in terms of the word length of $\gamma$ with respect to the generating set $\gamma_{1}, \ldots, \gamma_{p}$ : For example, write $\rho_{i}$ for $\rho\left(\gamma_{i}\right)$ and bound

$$
\begin{aligned}
d\left(\rho_{i} \rho_{j} P, P\right) & =d\left(\rho_{j} P, \rho_{i}^{-1} P\right) \\
& \leq d\left(\rho_{j} P, P\right)+d\left(P, \rho_{i}^{-1} P\right) \\
& =d\left(\rho_{j} P, P\right)+d\left(\rho_{i} P, P\right) \\
& \leq 2 \delta^{\prime} .
\end{aligned}
$$


It follows inductively that if $\gamma$ has word length $|\gamma| \leq k$ with respect to the generating set, then

$$
d(\rho(\gamma) P, P) \leq k \delta^{\prime}
$$

We construct an initial equivariant map $v$ which is piecewise constant on $\tilde{M}$, and we first consider the case of compact $M$ : Pick a compact fundamental domain $M_{0}$ for $M$, in $\tilde{M}$, so that its boundary has zero measure. Then the action of $\gamma \in \Gamma$ on $\tilde{M}$ moves $M_{0}$ to a different fundamental domain $\gamma M_{0}$, and these images define a partitioning of $\tilde{M}$ except on the zero-dimensional set formed by their boundaries. Define the piecewise constant function $v$ by the equivariant extension of $v\left(M_{0}\right)=P$ :

$$
v\left(\gamma M_{0}\right) \equiv \rho(\gamma) P .
$$

We define the (mollified) map $u(x)$ to be the average of $v$, taken over $B(x, 1)$. In the formalism of Proposition 2.5.2 take the measure space $\mathcal{M}$ to be the natural numbers $\mathbb{N}$. Let $\gamma_{1}, \gamma_{2}, \ldots$ be a denumeration of $\Gamma$ which extends $\gamma_{1}, \ldots, \gamma_{p}$. Define the mapping $f$ from $\mathbb{N}$ to $X$ by

$$
f(i)=\rho\left(\gamma_{i}\right) P .
$$

For $x \in M_{0}$ define the probability measure $\nu=\nu_{x}$ on $\mathbb{N}$ by

$$
\nu_{x}(i)=\frac{\mu\left(B(x, 1) \cap \gamma_{i}\left(M_{0}\right)\right)}{\mu(B(x, 1))} .
$$

(Here $\mu$ is the Riemannian volume measure on $\tilde{M}$ obtained by lifting the corresponding measure from $M$.) Define

$$
u(x)=\bar{f}_{\nu_{x}} .
$$

The map $u$ is $\Gamma$-equivariant because the measures $\nu_{x}$ are $\Gamma$-invariant, and because $f$ is equivariant with respect to the natural action of $\Gamma$ on $\mathbb{N}$.

Because $M_{0}$ is compact there is a finite bound $\mathrm{k}$ so that whenever the "translation" $\gamma M_{0}$ has points within distance one of $M_{0}$, then the word length $|\gamma| \leq k$. It follows that (2.6iv) holds with this choice $k$, for any such $\gamma$. Hence

$$
d(u(x), f(i)) \leq 2 k \delta^{\prime}
$$


whenever $\gamma=\gamma_{i}$ is as above. This is because all such $f(i)$ are within $k \delta^{\prime}$ of $P$ so the center of mass $u(x)$ (which depends only on these values $f(i)$ ) is also within the $k \delta^{\prime}$ ball about $P$ (e.g. Proposition 2.5.4).

If $x, y \in \tilde{M}$ it is clear that we also have the estimate

$$
\sum_{i}\left|\nu_{x}(i)-\nu_{y}(i)\right| \leq C_{1}|x-y|
$$

for some constant $C_{1}$ depending only on $M$.

We now apply Proposition 2.5.2 in the case $f=h, \nu=\nu_{y}$ and $\nu^{\prime}=\nu_{x}$. The first term in the resulting estimate is zero, we ignore the second one since it is non-positive, and we estimate the final one using $(2.6 \mathrm{v}, 2.6 \mathrm{vi})$ above. The result is the inequality

$$
d^{2}(u(x), u(y)) \leq 2 d(u(x), u(y)) 2 k \delta^{\prime} C_{1}|x-y|,
$$

which implies the desired uniform Lipschitz constant for $u$. (Since the Lipschitz constant of $u$ is $\Gamma$-invariant it suffices to estimate it on $M_{0}$.)

For non-compact $M$ the proof is essentially the same, except now the fundamental domain $M_{0}$ is only locally compact. We replace the global constant $k$ in (2.6vii) with a number $k(x)$ which measures the maximum word length $|\gamma|$ over $\gamma$ satisfying

$$
B(x, 1) \cap \gamma M_{0} \neq \emptyset .
$$

The constant $C_{1}$ must now be chosen to depend on $x$ as well, so that (2.6vi) holds for all $y \in B(x, 1)$. The inequality then holds for all $y$ if $C_{1}$ is taken to be $\geq 2$. (If $M$ had sectional curvature bounded from below then $C_{1}$ could still be chosen globally.) The result of these modifications is the inequality

$$
d^{2}(u(x), u(y)) \leq 2 d(u(x), u(y)) 2 k(x) \delta^{\prime} C_{1}(x)|x-y|
$$

for all $x, y \in \tilde{M}$. This proves Proposition 2.6.1.

Remark 2.6.2. Assume the configuration of Proposition 2.6.1. If we select a "small" closed set $\mathcal{C}_{\varepsilon}$ in $M$, by which we mean one that lifts to a compactly contained subset of the interior of $M_{0}$, then we can find an equivariant map $u$ which is piecewise constant on each lift of $\mathcal{C}_{\varepsilon}$, and which has the same Lipschitz constant control as that given in Theorem 2.6.1. The way to construct this function in terms of our previous calculations is to first scale the domain metric 
so that the lift of $\mathcal{C}_{\varepsilon}$ is distance at least 1 away from $\partial M_{0}$. In this case the function $u$ constructed in the proposition will have the desired properties.

For general complete $M$ it is not clear whether the maps $u$ constructed in Proposition 2.6.1 will have finite energy; the calculation for a particular $M$ depends on an interplay between the deterioration of the Lipschitz constant and the decay of the volume, as one approaches $\infty$ on $M$.

Even when the set of candidate maps for the equivariant problem (without boundary) can be shown to be non-empty, there is no Poincaré inequality so the question of convergence is much more delicate than it was for the Dirichlet problem. For example, it is possible for a minimizing sequence to approach $\infty$ in $X$. In the case of non-locally compact target it may even happen that a sequence which is uniformly bounded has no convergent subsequence. It is true and somewhat surprising, however, that the sequence of tensors $\pi$ for any minimizing sequence does converge, to a unique limit tensor:

Proposition 2.6.3. Let $M$ be a metrically complete Riemannian manifold, possibly with compact Lipschitz boundary $\partial M$, and with fundamental group $\pi_{1}(M)=\Gamma$. let $X$ be an NPC space, and let $\rho: \Gamma \rightarrow i s o m(X)$ be a homomorphism. If the corresponding set $\mathcal{S}$ of of equivariant $(p=2)$ Sobolev maps is non-empty, then for any energy minimizing sequence $\left\{v_{i}\right\} \subset \mathcal{S}$ we have

$$
\lim _{i, j \rightarrow \infty} \int_{M} \int_{S^{n-1}}||\left(v_{i}\right)_{*}(\omega)|-|\left(v_{j}\right)_{*}(\omega)||^{2} d \sigma(\omega) d \mu(x)=0 .
$$

In particular, there is a unique integrable tensor $\pi$ so that

$$
\lim _{i \rightarrow \infty} \int_{M} \int_{S^{n-1}}\left|\pi(\omega, \omega)-\pi_{v_{i}}(\omega, \omega)\right| d \sigma(\omega) d \mu(x)=0
$$

Proof. For two maps $u, v$ and their midpoint map $w$, we apply quadrilateral comparison (2.1iv) with $t=\frac{1}{2}$ and (for the first time in this paper) $\alpha=0$ :

$$
\begin{aligned}
d^{2}(w(x), w(y)) \leq & \frac{1}{2} d^{2}(u(x), u(y))+\frac{1}{2} d^{2}(v(x), v(y)) \\
& -\frac{1}{4}[d(u(x), u(y))-d(v(x), v(y))]^{2}
\end{aligned}
$$


Multiplying this inequality by a non-negative $f$ having support in the interior of $M$, ball-averaging as in (1.2vii), and letting $\varepsilon \rightarrow 0$, yields

$$
\begin{gathered}
\limsup _{\varepsilon \rightarrow 0} \frac{n+2}{4 \omega_{n}} \int_{|x-y|<\varepsilon} \int_{|c|} f(x)\left(\frac{d(u(x), u(y))}{\varepsilon}-\frac{d(v(x), v(y))}{\varepsilon}\right)^{2} \frac{d \mu(x) d \mu(y)}{\varepsilon^{n}} \\
\leq \frac{1}{2} E^{u}(f)+\frac{1}{2} E^{v}(f)-E^{w}(f) .
\end{gathered}
$$

(We have normalized the functionals $E^{u}(\bullet)$ to be consistent with our total energy definition (1.10v).) Using the techniques of Chapter 1 it is possible to show that the expression on the left of (2.6ix) approaches $\frac{1}{4 \omega_{n}}$ times

$$
\int_{S^{n-1}} \int_{M} f(x)|| u_{*}(\omega)|-| v_{*}(\omega)||^{2} d \mu(x) d \sigma(\omega)
$$

as $\varepsilon \rightarrow 0$. We leave the details to the reader, but sketch the idea: Use a partition of unity to approximate the integral in (2.6ix) with a sum of ones involving $\varepsilon$ directional energies, so that the new limits are $\Omega \times S^{n-1}$. Then use the fact that for directional energies, the $p=1$ approximate energy density functions of $p=2$ Sobolev maps converge in $L_{l o c}^{2}$ to the $p=1$ energy functions. The claimed limit will then follow from the Lebesgue dominated convergence theorem.

Pick an increasing sequence $\left\{f_{k}\right\}$ with compact support in the interior of $M$, so that the $f_{k}$ converge to the constant 1 . The right side of (2.6ix) converges to the number

$$
\frac{1}{2} E^{u}+\frac{1}{2} E^{v}-E^{w}
$$

We may therefore deduce the first limit claim of Proposition 2.6.3 by choosing $u=v_{i}, v=v_{j}$ in (2.6ix), for the functions $\left\{f_{k}\right\}$. The second claim follows from the fact that

$$
\left|\pi_{u}(\omega, \omega)-\pi_{v}(\omega, \omega)\right| \leq|| u_{*}\left(\omega|-| v_{*}(\omega)|\cdot||| u_{*}(\omega)|+| v_{*}(\omega)||\right.
$$

and the Cauchy-Schwartz inequality.

In case one is attempting to understand the behavior of a minimizing sequence, it is helpful to know that the modulus of continuity is under control. The next theorem allows one to construct Lipschitz minimizing sequences. 
The idea is to modify a given minimizing sequence as follows: Use its values as boundary data for a family of Dirichlet problems, and then use the averaging techniques of $\S 2.5$ to piece these Dirichlet problem solutions into a Lipschitz sequence which is still minimizing. In spirit this is like the Perron method for constructing harmonic functions, but the technical ideas here are quite different since they are not based on the maximum principle.

Theorem 2.6.4. Let $M$ be a complete Riemannian manifold with finite volume (and without boundary), and let $X$ be an NPC metric space. Let $\rho: \Gamma \rightarrow$ isom $(X)$ be a representation of $\pi_{1}(M)=\Gamma$. If $M$ is compact there is an energy minimizing $\rho$-equivariant sequence $\left\{u_{i}\right\}$ with the property that all the $u_{i}$ are (uniformly) Lipschitz continuous. In fact, there exists a $C$ depending only on $M$ so that the Lipschitz constants of each $u_{i}$ can be bounded by

$$
C \delta(P)
$$

where $\delta$ is the displacement function (2.6iii) and $P$ is any point in $X$.

If $M$ is complete (but not compact), suppose the set of finite-energy $\rho-$ equivariant maps from $\tilde{M} \rightarrow X$ is non-empty, and that there exists one of energy $E<\infty$. Then there is an equivariant minimizing sequence $\left\{u_{i}\right\}$ : $\tilde{M} \rightarrow X$, so that for any compact subset $K \subset M$ and $i$ sufficiently large (depending on $K$ ), the $u_{i}$ are Lipschitz continuous on (the lift to $\tilde{M}$ of) $K$, with pointwise Lipschitz constants bounded by $C(x) E^{\frac{1}{2}}$. Here $C(x)$ is a locally bounded function depending only on $M$.

Proof. First treat the case of compact $M$. Start by picking a finite covering of it with (say) balls, $\left\{B^{j}\right\}_{j=1, \ldots, m}$. Pick the balls small enough so that for any $x \in M$ the set

$$
\bigcup_{j \mid x \in B^{j}} B^{j}
$$

is simply connected. Pick a subordinate partition of unity, $\left\{\eta^{j}\right\}$, and compact subsets $Z^{j} \subset B^{j}$ so that the support of each $\eta^{j}$ is contained in the interior of $Z^{j}$. Lift the functions $\eta^{j}$, and the sets $B^{j}, Z^{j}$ to invariant functions $\tilde{\eta}^{j}$ and invariant sets $\tilde{B}^{j}, \tilde{Z}^{j}$ on $\tilde{M}$. 
By Proposition 2.6.1 the set of admissible maps is non-empty, and the infimum of their energies $E^{0}$ is bounded by

$$
E^{0} \leq C \delta(P)^{2}
$$

where $C$ depends only on $M$. Now, let a minimizing sequence $\left\{v_{i}\right\}$ be given, with energies $E^{v_{i}} \rightarrow E^{0}$. In each $\tilde{B}^{j}$ use the (equivariant) traces of $v_{i}$ as Dirichlet data, and apply Theorem 2.2 to construct equivariant harmonic maps $u_{i}^{j}$. Extend $u_{i}^{j}$ outside $\tilde{B}^{j}$, by defining $u_{i}^{j}=v_{i}$ there. By Theorem 1.12.3 it follows that

$$
E^{u_{i}^{j}} \leq E^{v_{i}}
$$

Now define the equivariant sequence $\left\{u_{i}\right\}$ on $\tilde{M}$ by

$$
u_{i}(x)=\sum_{i=1}^{m} \tilde{\eta}^{j}(x) u_{i}^{j}(x) .
$$

In the formalism of Proposition 2.5.2, this means that for the measure space $\mathcal{M}=\{1, \ldots, m\}$ and for $x \in \tilde{M}$ we assign a measure $\nu_{x}$ with values

$$
\nu_{x}(j)=\tilde{\eta}^{j}(x)
$$

the map $f:\{1, \ldots, m\}: \rightarrow X$ given by

$$
f(j)=u_{i}^{j}(x)
$$

and define $u_{i}(x)$ to be the center of mass $\bar{f}_{\nu_{x}}$.

Proposition 2.5.2 allows us to compare $u_{i}(x)$ to $u_{i}(y)$ for $y$ near $x$. Defining $\nu_{y}$ in terms of the values $\tilde{\eta}^{j}(y)$ and $h$ in terms of the values $u_{i}^{j}(y)$, we deduce (2.6xiii)

$$
\begin{aligned}
d^{2}\left(u_{i}(x), u_{i}(y)\right) \leq & \sum_{j=1}^{m} \tilde{\eta}^{j}(x) d^{2}\left(u_{i}^{j}(x), u_{i}^{j}(y)\right) \\
& +2 d\left(u_{i}(x), u_{i}(y)\right) \sum_{j=1}^{m} d\left(u_{i}^{j}(y), u(y)\right)\left|\tilde{\eta}^{j}(y)-\tilde{\eta}^{j}(x)\right| .
\end{aligned}
$$

The following limit statement, which we digress to prove, quantifies the sense in which the second term in the right side of (2.6xiii) becomes negligible as 
$i \rightarrow \infty:$

(2.6xiv)

$$
\lim _{i \rightarrow \infty} \sup _{\substack{j \in\{1, \ldots, m\} \\ y \in \tilde{Z}^{j}}} d\left(u_{i}^{j}(y), u_{i}(y)\right)=0 .
$$

Since $u_{i}(y)$ is an average of the $u_{i}^{j}(y)$ for which $y \in \tilde{Z}^{j}$, (2.6xiv) follows from Proposition 2.5.4 and the equation

$$
\lim _{i \rightarrow \infty} \sup _{\substack{j, l \in\{1, \ldots, m\} \\ y \in \tilde{Z}^{j} \cap \tilde{Z}^{l}}} d\left(u_{i}^{j}(y), u_{i}^{l}(y)\right)=0 .
$$

We show $(2.6 \mathrm{xv})$ : There is a $\delta_{0}>0$ depending on the sets $Z^{j}$ so that whenever $y \in \tilde{Z}^{j}$, the ball $B\left(y, 2 \delta_{0}\right) \subset \tilde{B}^{j}$. From the interior Lipschitz continuity (Theorem 2.4.6) and from (2.6xi) we see that for $|z-y|<\delta_{0}$ the Lipschitz constant of $L_{i}^{j}(z)$ of $u_{i}^{j}(z)$ is uniformly bounded (independently of $y, Z^{j}$ and large $i)$,

$$
L_{i}^{j}(z) \leq L \delta(P)^{2} \equiv L^{\prime}
$$

(for some constant $L$ depending on $M$ and $E^{0}$ ). Thus for $y \in \tilde{Z}^{j} \cap \tilde{Z}^{l}$ and $|z-y|<\delta$ the triangle inequality yields

$$
d\left(u_{i}^{j}(z), u_{i}^{l}(z)\right) \geq d\left(u_{i}^{j}(y), u_{i}^{l}(y)\right)-2 L^{\prime} \delta .
$$

Integrate over $B(y, \delta)$ to get

(2.6xvii) $\int_{B(y, \delta)} d^{2}\left(u_{i}^{j}(z), u_{i}^{l}(z)\right) d \mu(z) \geq C \delta^{n}\left[d\left(u_{i}^{j}(y), u_{i}^{l}(y)\right)-2 L^{\prime} \delta\right]^{2}$.

The function $d\left(u_{i}^{j}, u_{i}^{l}\right)$ defined on $M$ equals zero outside $B^{j} \cup B^{l}$, so we can bound the left side of the above inequality via the Poincaré inequality

$$
\int_{B^{j} \cup B^{l}} d^{2}\left(u_{i}^{j}, u_{i}^{l}\right) d \mu \leq C \int_{B^{j} \cup B^{l}}\left|\nabla d\left(u_{i}^{j}, u_{i}^{l}\right)\right|^{2} d \mu .
$$

By the energy convexity statement (2.6ii) and by (2.6xii) we have the bound

$$
\int_{B^{j} \cup B^{l}}\left|\nabla d\left(u_{i}^{j}, u_{i}^{l}\right)\right|^{2} d \mu \leq 4\left(E^{v_{i}}-E^{0}\right)
$$

for the right side of (2.6xviii). The limit claim $(2.6 \mathrm{xv})$ follows by combining (2.6xvii), (2.6xviii), and (2.6xix) for arbitrarily small $\delta$. 
We now return to the estimate (2.6xiii) to show the uniform Lipschitz continuity of the functions $u_{i}$, for $i$ large. By (2.6xiv) and the geometric-arithmetic mean inequality we may pick $i$ large enough so that the second term on the right of (2.6xiii) is bounded above by

$$
\frac{1}{2} d^{2}\left(u_{i}(x), u_{i}(y)\right)
$$

independently of $x, y$. Thus

$$
d^{2}\left(u_{i}(x), u_{i}(y)\right) \leq 2 \sum_{j=1}^{m} \tilde{\eta}^{j}(x) d^{2}\left(u_{i}^{j}(x), u_{i}^{j}(y)\right) .
$$

Applying the interior Lipschitz continuity estimate (2.6xvi) for the harmonic map $u_{i}^{j}$ then gives

$$
d^{2}\left(u_{i}(x), u_{i}(y)\right) \leq C \delta(P)^{2}
$$

for some universal constant $C$ depending only on $M$. By removing a finite number of the $\left\{u_{i}\right\}$ from our sequence, we deduce the Lipschitz control claimed in Theorem 2.6.4.

To show that $\left\{u_{i}\right\}$ is minimizing we also use (2.6xiii). Applying Young's inequality to the second term on its right side gives

$$
\begin{aligned}
(1-\delta) d^{2}\left(u_{i}(x), u_{i}(y)\right) \leq & \sum_{j=1}^{m} \eta^{j}(x) d^{2}\left(u_{i}^{j}(x), u_{i}^{j}(y)\right) \\
& +\frac{1}{\delta}\left(\sum_{j} d^{2}\left(u_{i}^{j}(y), u_{i}(y)\right)\right)\left(\sum_{j}\left|\eta^{j}(y)-\eta^{j}(x)\right|^{2}\right) .
\end{aligned}
$$

Averaging with respect to $|y-x| \leq \varepsilon$, integrating over $M$, letting $\varepsilon \rightarrow 0$, and recalling that $E^{u}(f)$ refers to the value of the linear functional $E^{u}(\bullet)$ applied to $f$, the above inequality implies

$$
(1-\delta) E^{u_{i}} \leq \sum_{j} E^{u_{i}^{j}}\left(\eta^{j}\right)+\frac{C}{\delta} o_{i}(1)
$$

(The $o_{i}(1)$ terms approaches zero as $i \rightarrow \infty$, by (2.6xiv).) Because the $\left\{\eta^{j}\right\}$ form a partition of unity, and because $E^{u}(\bullet)$ is linear,

$$
(1-\delta) E^{u_{i}} \leq E^{v_{i}}+\sum_{j}\left(E^{u_{i}^{j}}\left(\eta^{j}\right)-E^{v_{i}}\left(\eta^{j}\right)\right) \quad+\frac{C}{\delta} o_{i}(1) .
$$


Appealing to Proposition 2.6.3 we deduce that the sum term in the above inequality approaches zero as $i \rightarrow \infty$, so

$$
\limsup _{i \rightarrow \infty}(1-\delta) E^{u_{i}} \leq E^{0}
$$

Hence $\left\{u_{i}\right\}$ is minimizing, since $\delta$ is arbitrary. Thus the proof of Proposition 2.6.4 is complete in case $M$ is compact.

The case of non-compact $M$ is a modification of the above argument. For a base-point $x_{0} \in M$ write

$$
K_{m}=\left\{x \in M:\left|x-x_{0}\right| \leq m\right\} .
$$

Pick a countable covering of $M$ by balls $\left\{B^{j}\right\}_{j \in \mathbb{N}}$ so that for an increasing sequence $\left\{j_{m}\right\}_{m \in \mathbb{N}}$ the finite collections

$$
\left\{B^{j}\right\}_{j=1, \ldots, j_{m}}
$$

cover $K_{m}$, and so that $j>j_{m}$ implies $B^{j} \cap K_{m-1}=\emptyset$. Pick a subordinate partition of unity $\left\{\eta^{j}\right\}$ and sets $Z^{j} \subset B^{j}$ as in the compact case, and denote their lifts to $\tilde{M}$ as before. For fixed $m \in \mathbb{N}$ we consider the finite partition of unity

$$
\left\{\eta^{j}\right\}_{j=1, \ldots, j_{m}} \cup\left\{1-\sum_{j=1}^{j_{m}} \eta^{j}\right\} .
$$

Given a minimizing sequence $\left\{v_{i}\right\}$ as before, we again do ball replacement in $\left\{\tilde{B}^{j}\right\}_{j=1, \ldots, j_{m}}$. Define the average map $u_{i}$ as before: one is now averaging over a set of $j_{m}+1$ points, the last of which is $v_{i}(x)$ itself. Because of the way they were constructed, $\left\{\eta^{j}\right\}_{j=1, \ldots, j_{m^{\prime}}}$ is a partition of unity for $K_{m_{\imath}^{\prime}-1}$, for each $m^{\prime} \leq m$. It follows that the limit statement (2.6xiv) still holds (uniformly) for $y \in \tilde{K}_{m^{\prime}-1}$. Therefore one is able to deduce a uniform Lipschitz bound $L$ for the functions $u_{i}$ restricted to $\tilde{K}_{m^{\prime}-1}$, when $i$ is large. It is of the form

$$
L \leq C_{m^{\prime}} E^{\frac{1}{2}}
$$

which is the claimed dependence. The proof that $\left\{u_{i}\right\}$ is minimizing proceeds as before, except now one replaces the pointwise estimate for $d\left(u_{i}^{j}(y), u_{i}(y)\right)$ (2.6xiv) with the weaker claim

$$
\lim _{i \rightarrow \infty} \int_{M} d^{2}\left(u_{i}^{j}(y), u_{i}(y)\right)=0, \quad j=1, \ldots, j_{m} .
$$


This follows from the triangle inequality and

$$
\lim _{i \rightarrow \infty} \int_{M} d^{2}\left(u_{i}^{j}(y), v_{i}(y)\right)=0 . \quad j=1, \ldots, j_{m} .
$$

Since $d\left(u_{i}^{j}(y), v_{i}(y)\right)=0$ outside of a compact subset of $M$ this last equality follows from the Poincaré inequality and energy convexity, i.e. the analogies of (2.6xvii) and (2.6xix). Thus, by a diagonalization with respect to the $K_{m}$ and the $v_{i}$ we are able to construct a suitable sequence $\left\{u_{i}\right\}$. The proof of Theorem 2.6.4 is complete.

Using minimizing sequences which have local modulus of continuity control enables one to reduce the question of global convergence to that of convergence at a point:

Proposition 2.6.5. Let $M$ be a complete Riemannian manifold, possibly with compact Lipschitz boundary $\partial M$. Let $\Gamma=\pi_{1}(M)$, and $\rho: \Gamma \rightarrow i \operatorname{som}(X)$ be a homomorphism. Let $\left\{u_{i}\right\}$ be an equivariant minimizing sequence which has local modulus of continuity control. That is, for each $x \in \tilde{M}$ we assume there is an (equivariant) function $\omega(x, r)\left(0 \leq r<r_{x}\right)$ which is monotone increasing in $r$, which satisfies $\omega(x, 0)=0$ and so that

$$
\sup _{i} \sup _{|x-z| \leq r} d\left(u_{i}(x), u_{i}(z)\right) \leq \omega(x, r) .
$$

Then the sequence $\left\{u_{i}\right\}$ converges (locally uniformly and hence in $L_{\text {loc }}^{2}$ ) to an equivariant harmonic map $u$ if and only if there exists an $x \in \tilde{M}$ at which the sequence of points $\left\{u_{i}(x)\right\}$ is convergent.

Proof. The reason this proposition is true is the energy convexity statement (2.6ii) which implies

$$
\int_{M}\left|\nabla d\left(u_{i}, u_{j}\right)\right|^{2} d \mu \rightarrow 0 .
$$

Let $x \in \tilde{M}$ be a point of convergence, $\left\{u_{i}(x)\right\} \rightarrow P$. By the modulus of continuity estimate and the triangle inequality it follows that

$$
\limsup _{i, j \rightarrow \infty} \sup _{|z-x| \leq r} d\left(u_{i}(z), u_{j}(z)\right) \leq 2 \omega(r)
$$


(where we have written $\omega(r)$ for $\omega(x, r)$. Thus for $i, j$ sufficiently large, the function

$$
d\left(u_{i}(z), u_{j}(z)\right)-3 \omega(r)
$$

is negative on the set $|z-x|=r$. For such $i, j$ we may apply the Poincaré inequality for compact exterior regions to $B(x, r)$ :

(2.6xxiii)

$$
\int_{B(x, R)}\left[\left(d\left(u_{i}(z), u_{j}(z)\right)-3 \omega(r)\right)^{+}\right]^{2} d \mu(z) \leq C_{r, R, x} \int_{B(x, R)}\left|\nabla d\left(u_{i}, u_{j}\right)\right|^{2} d \mu .
$$

By (2.6xxii) the integral on the right converges to zero as $i, j \rightarrow \infty$, so we deduce from (2.6xxiii) and the modulus of continuity control that

$$
\limsup _{i, j \rightarrow \infty} d\left(u_{i}(z), u_{j}(z)\right) \leq 3 \omega(r)
$$

for all $z \in B(x, R)$. Since $R$ and $r$ are arbitrary, it follows that the sequence $\left\{u_{i}\right\}$ converges everywhere. Because of the modulus of continuity control this convergence is uniform on lifts of compact subsets of $M$. In particular, the $u_{i}$ converge locally in $L^{2}$ to an equivariant map $u$, so semicontinuity (Theorem 1.6.1) applies and the map $u$ is harmonic.

Remark 2.6.6. Compare the results of the last three propositions. If the class of equivariant Sobolev maps is nonempty, and if $X$ is locally compact, then the minimizing sequences of 2.6.4 either converge to the ideal boundary (at $\infty$ ) of $X$, or a subsequence converges to a harmonic map. It follows from energy convexity (2.6ii)(2.5iii) that all harmonic maps are contained in a (possibly) multi-parameter family of "parallel" harmonic maps. (And from 2.6 .3 they all induce the same tensor $\pi$.) A very interesting and important (unanswered) problem is to understand when harmonic maps are actually unique (up to domain or range isometries).

Even if the minimizing sequence from 2.6.4 does not have a convergent subsequence in $X$, it is still true that the real-valued functions $d\left(u_{i}(x), u_{i}(y)\right)$ have subsequences which converge to $\Gamma$-invariant distance functions $d(x, y)$ defined on $\tilde{M} \times \tilde{M}$. In our sequels to this paper we will show that the infinitesimal metric induced by any such limit $d$ is the unique tensor $\pi$ of 2.6.3. In fact, $d$ actually arises as an induced distance for a map to a (limit) NPC space 
constructed from convex subsets of $X$. We call these limit $d$ 's "harmonic", and their structure is the focus of much of our later work.

One way to guarantee existence of a limit is to impose Dirichlet conditions. We will make use of the following result in our sequels:

Proposition 2.6.6. Consider the configuration of Proposition 2.6.1 and a small set $C_{\varepsilon}$ in $M$, as defined in Remark 2.6.2. Let there be a $\rho$ - equivariant Sobolev map $\psi$ from $\tilde{M}$ to $X$. Then there exists a unique locally Lipschitz $\rho$-equivariant harmonic map $u: \tilde{M}-\tilde{C}_{\varepsilon}: \rightarrow X$ with trace $\psi$ on $\partial \tilde{C}_{\varepsilon}$. If the boundary is smooth and if $\psi$ extends to be $C^{\alpha}(0<\alpha<1)$ up to the boundary, then so does $u$.

Proof. By energy convexity (2.6ii) and Poincaré inequalities for exterior regions (to $\partial C_{\varepsilon}$ ) a minimizing sequence converges in $L_{l o c}^{2}$, to an equivariant harmonic map. The interior regularity follows from Theorem 2.4.6 and the boundary regularity follows from [Se] (See Remark 2.4.7.)

2.7. Homotopy problems. As a final application of the techniques in this paper we generalize the classical Eells-Sampson harmonic map theory to the case of metric space targets. For technical simplicity we assume our domain manifold $M$ is compact. Let $N$ be a metric space with universal cover $X$ which is NPC. We will say that a continuous map $u: M \rightarrow N$ is harmonic if it is locally energy minimizing. Precisely, each $x \in M$ is to have a neighborhood so that all continuous comparison maps which agree with $u$ outside this neighborhood have no less energy. We prove:

Theorem 2.7.1. Let $M$ be as above, with $\partial M=\emptyset$. Let $N$ be compact, and let $f: M \rightarrow N$ be a continuous map. Then there exists a Lipschitz harmonic map $u: M \rightarrow N$ which is homotopic to $f$.

Proof. For fixed $x \in M$ the $f$ induces the homomorphism $f_{*}: \pi_{1}(M)_{x} \rightarrow$ $\pi_{1}(N)_{f(x)}$. We may lift $f$ to a map $\tilde{f}: \tilde{M} \rightarrow X$, so $\tilde{f}$ is $f_{*}$ equivariant. Use Proposition 2.6.1 to construct a finite energy $f_{*}$-equivariant map, and Theorem 2.6.4 to construct a uniformly Lipschitz minimizing sequence $\left\{\tilde{u}_{i}\right\}$. Because $X$ is NPC one may use geodesic homotopies to deduce that all continuous $f_{*}$-equivariant maps are homotopic. (The continuity of the homotopies 
follows from the quadrilateral comparisons of [Re], i.e. Theorem 2.1.2.) Hence the maps $\tilde{u}_{i}$ are equivalently homotopic to $\tilde{f}$, and we deduce that the projections $u_{i}$ are homotopic to $f$. Since the $u_{i}$ are uniformly Lipschitz continuous, a subsequence converges uniformly to a limit map $u$, which is therefore also homotopic to $f$.

It remains to show that the map $u$ is harmonic. Let $x \in M$ and let $\mathcal{O}$ be a simply connected neighborhood of $x$ with Lipschitz boundary. We claim that $u$ is minimizing with respect to continuous comparison functions which agree with $u$ outside $\mathcal{O}$. Since $\mathcal{O}$ is simply connected the map $\left.u\right|_{\mathcal{O}}$ lifts to a map $\tilde{u}$ from $\mathcal{O}$ to $X$, and we are reduced to showing that this map $\tilde{u}$ solves the Dirichlet problem of $\S 2.2$.

For any $\varepsilon>0$ and $i$ sufficiently large, we may choose the lifting $\tilde{u}$ so that

$$
\begin{gathered}
\sup _{z \in \mathcal{O}} d\left(\tilde{u}_{i}(z), \tilde{u}(z)\right)<\varepsilon \\
\int_{\mathcal{O}} d^{2}\left(\tilde{u}_{i}, \tilde{u}\right) d \mu<\varepsilon .
\end{gathered}
$$

Let $\tilde{v}_{i}$ be the solution to the Dirichlet problem on $\mathcal{O}$, with boundary data $u_{i}$. Let $\tilde{v}$ be the solution with data $\tilde{u}$. Since $d^{2}\left(\tilde{v}_{i}, \tilde{v}\right)$ is subharmonic (Remark 2.4.3), $d\left(\tilde{v}_{i}, \tilde{v}\right)$ attains its maximum on $\partial \mathcal{O}$, so is bounded by the maximum of $d\left(u_{i}, u\right)$ there. In particular we see that for sufficiently large $i$

$$
\int_{\mathcal{O}} d^{2}\left(\tilde{v}_{i}, \tilde{v}\right) d \mu<\varepsilon
$$

Finally, from energy convexity (2.6ii) and the Poincaré inequality for $\mathcal{O}$, we have

$$
\int_{\mathcal{O}} d^{2}\left(\tilde{u}_{i}, \tilde{v}_{i}\right) d \mu<\varepsilon
$$

for $i$ large. Combining the three estimates and using the $L^{2}$ triangle inequality, we deduce

$$
\int_{\mathcal{O}} d^{2}(\tilde{u}, \tilde{v}) d \mu<9 \varepsilon
$$

Hence $\tilde{u}=\tilde{v}$ and so $u$ is harmonic. 
If one is considering the Dirichlet homotopy problem, then one need not assume local compactness on the target space:

Theorem 2.7.2. Let $M$ be a compact manifold with smooth boundary, and let $N$ be a complete metric space with NPC universal cover $X$. Let $f: M \rightarrow N$ be a continuous $p=2$ Sobolev map, with the property that its trace on $\partial M$ is $C^{\alpha}$ for some $0<\alpha<1$. Then there exists a unique globally minimizing harmonic map $u: M \rightarrow N$ homotopic to $f$ and with the same boundary values. $u$ is Lipschitz continuous in the interior of $M$ and extends to be $C^{\alpha}$ up to the boundary.

Proof. We may lift $f$ to an $f_{*}$ equivariant map $\tilde{f}$ as in the previous theorem. Construct the homomorphism $f_{*}$ from $\pi_{1}(M)_{x}$ to $\pi_{1}(N)_{f(x)}$ for a base point $x \in \partial M$. By hypothesis the lifing is a finite energy map (in the sense of $\S 2.6)$. It follows from energy convexity (2.6ii) and the $(M, \partial M)$ Poincaré inequality that a minimizing sequence of $f_{*}$-equivariant maps having the same boundary data as $\tilde{f}$ converges in $L^{2}$ (in the sense of $\S 2.6$ ), to a unique harmonic equivariant map. This map is locally Lipschitz and extends to be $C^{\alpha}$ up to the boundary (by Theorem 2.4.6 and the results of [Se]). It follows (via geodesic homotopy, as in the previous theorem) that its projection $u$ is homotopic to $f$. By our choice of basepoint we see that a continuous map $v: M \rightarrow N$ which is homotopic to $f$ relative to $\partial M$ lifts to an $f_{*}$ - equivariant map, so it follows that $u$ is minimizing relative to all such maps.

\section{REFERENCES}

[CZ] Corlette, K. and Zimmer, R.J., Super Rigidity for cocycles and hyperbolic geometry, preprint.

[ES] Eells, J. and Sampson, J.H., Harmonic mappings of Riemannian Manifolds, Amer.J. Math., 86 (1964), 109-160.

[Fe] Federer, H., Geometric Measure Theory, Grundlehrer der mathematische Wissenschafte Band 153 Springer-Verlag NY 1969.

[Gi] Giusti, E., Minimal Surfaces and Functions of Bounded Variation, Birkhäuser,Boston 1984.

[GS] Gromov, M. and Schoen, R., Harmonic maps into singular spaces and p-adic superrigidity for lattices in groups of rank one, IHES Publications Mathématique 76 (1992), 165-246.

[GT] Gilbarg, D. and Trudinger, N. S., Elliptic Partial Differential Equations of Second Order, Second Edition, Srpinger-Verlag, New York 1983. 
[Ha] Hamilton, R., Harmonic maps of manifolds with boundary, Lecture Notes in Math., 471 Springer, 1975.

[Mo] Morrey, C.B., Multiple Integrals in the calculus of variations, Springer-Verlag NY 1966.

[Re] Reshetnyak, Y.G., Nonexpanding maps in a space of curvature no greater than $K$, Siberian Math. Journ., 9 (1968), 918-927.

[Ru] Rudin, W., Real and Complex Analysis, second edition, McGraw-Hill, New York 1974.

[Sch] Schoen, R., Analytic aspects of the harmonic map problem, Math Sci. Res. Inst. Publ. vol. 2, Springer Berlin, 1984 321-358.

[Se] Serbinowski, T., Boundary regularity of energy minimizing maps, preprint.

[Wa] Wald, A., Begründung einer koordinatenlosen Differentialgeometrie der Flächen, Ergebnisse eines mathematischen Kooloquiums, 7 (1935), 2-46.

[Zi] Zimmer, R., Strong rigidity for ergodic actions of semisimiple Lie groups, Ann. of Math. 112 (1980), 511-529.

UNIVERSITY OF UTAH, U. S. A.

STANFORD UNIVERSITY, U. S. A.

RECEIVED MAY 25, 1993. 